\title{
Total Synthesis of RK-397
}

Scott E. Denmark and Shinji Fujimori

Department of Chemistry, University of Illinois Urbana-Champaign, Urbana, Illinois 61801

\section{SUPPORTING INFORMATION}

Table of Contents

General Experimental

Literature Preparations

Synthesis Scheme

Experimental Procedures and Configurational Assignments

Comparison of Natural and Synthetic RK-397

References
Page

S1

S3

S3

S5

S44

$S 50$

\section{General Experimental}

${ }^{1} \mathrm{H},{ }^{13} \mathrm{C}$ and ${ }^{31} \mathrm{P}$ NMR spectra were recorded on Varian Unity-400 $\left(400 \mathrm{MHz}{ }^{1} \mathrm{H}, 100\right.$ $\left.\mathrm{MHz}{ }^{13} \mathrm{C}, 162 \mathrm{MHz}{ }^{31} \mathrm{P}\right)$, Unity-500 (500 MHz $\left.{ }^{1} \mathrm{H}, 125 \mathrm{MHz}{ }^{13} \mathrm{C}, 202 \mathrm{MHz}{ }^{31} \mathrm{P}\right)$ and Varian Unity Inova $500\left(500 \mathrm{MHz}{ }^{1} \mathrm{H}\right)$ spectrometers in deuterochloroform unless otherwise stated. Data are reported in the following order: chemical shift in ppm $(\delta)$; multiplicities are indicated (br (broadened), s (singlet), d (doublet), t (triplet), q (quartet), m (multiplet)); coupling constants, $J$, are reported in hertz $(\mathrm{Hz})$; integration is provided; and assignment is indicated. ${ }^{1} \mathrm{H}$ and ${ }^{13} \mathrm{C}$ NMR assignments are corroborated by 2D experiments (HETCOR and COSY). Spectra are available on request from denmark@scs.uiuc.edu. Mass spectrometry was performed by the University of Illinois Mass Spectrometry Center. Electron impact (EI) spectra were performed on a Micromass 70-VSE spectrometer, chemical ionization (CI) spectra were obtained on a Micromass 70-VSE spectrometer using methane as the carrier gas, field ionization (FI) spectra were obtained from Micromass 70-VSE spectrometer, electrospray ionization (ESI) spectra were obtained from Micromass Q-TOF spectrometer. Data are reported in the form of $\mathrm{m} / \mathrm{z}$ (intensity relative to base peak $=100)$. Infrared spectra $(I R)$ were recorded on a Mattson Galaxy 5020 
spectrophotometer. Peaks are reported in $\mathrm{cm}^{-1}$ with indicated intensities: s (strong, $67-100 \%$ absorption), m (medium, $34-66 \%$ ), w (weak, $0-33 \%$ ). UV/Vis spectrum was obtained from HP8453 spectrophotometer. Optical rotations were measured on a Jasco DIP-360 digital polarimeter and are reported in the standard format for $[\alpha]$. The CD spectrum was obtained with Jasco J-720 spectropolarimeter. Elemental analyses were performed by the University of Illinois Microanalytical Service Laboratory.

Analytical thin-layer chromatography was performed on Merck silica gel plates with F254 indicator. Visualization was accomplished by UV light, iodine, potassium permanganate solution $\left(\mathrm{KMnO}_{4}\right)$, phosphomolybdic acid stain(PMA), p-anisaldehyde stain or dinitrophenylhydrazine (DNP) solution. Column chromatography was performed by the method of Still ${ }^{1}$ with 32-63 mm silica gel (Merck). Bulb-to-bulb distillations were performed on a Büchi GKR-50 Kugelrohr and boiling points (bp) correspond to uncorrected, air bath temperatures. Analytical supercritical fluid chromatography (SFC) was performed on a Berger Instruments packed-column SFC with built in photometric detector. Analytical columns used were Daicel Chiralpak $\mathrm{AD}$ and $\mathrm{AS}$, Chiralcel OJ and OD and Regis $(R, R)$-Welk-01. Analytical and preparative HPLC were performed on Rainin Model SD 200 equipped with UV absorbance detector (Model UV 1) at a wavelength of $360 \mathrm{~nm}$. The products were analyzed and purified on Vydac C-18 (analytical column, 250 x $4.6 \mathrm{~mm}$; preparative column, 250 x $22 \mathrm{~mm}$ ). Products were eluted with 40/60 to 20/80 water/methanol with flow rates of $1 \mathrm{~mL} / \mathrm{min}$ for analytical and 8 $\mathrm{mL} / \mathrm{min}$ for preparative runs. Melting points $(\mathrm{mp})$ were determined in sealed capillary tubes on a Thomas-Hoover melting point apparatus and are corrected.

All reactions were performed in oven $\left(140{ }^{\circ} \mathrm{C}\right)$ and/or flame-dried glassware under an atmosphere of dry argon, unless otherwise stated. All reaction temperatures correspond to internal temperatures measured by Teflon-coated thermocouples unless otherwise noted. Solvents for extraction and chromatography were technical grade and distilled from the indicated drying agents: dichloromethane $\left(\mathrm{CH}_{2} \mathrm{Cl}_{2}\right)$, pentane, hexane: $\mathrm{CaCl}_{2}$; ethyl acetate (EtOAc): $\mathrm{K}_{2} \mathrm{CO}_{3}$; acetone and methanol $(\mathrm{MeOH})$ were reagent grade. $n$-Butyllithium was titrated according to the method of Gilman. " "Brine" refers to a saturated solution of NaCl. Polymerbound piperidine (200 - $400 \mathrm{mesh}, 3.0 \mathrm{mmol} / \mathrm{g}$ loading, 1\% cross-lined with divinylbenzene) was purchased from Aldrich and used as received. 


\section{Literature Preparations}

The following compounds were prepared by literature methods: 3-benzyldimethylsilylpropargyl alcohol $\quad(7)^{3}, \quad$ 1-tert-butylsilyloxy-1-ethoxy-1,3-butadiene $\quad(\mathbf{6})^{4}, \quad(2 R, 3 R)-3-p$ methoxybenzyloxy-2,4-dimethylpentanal $\mathbf{( 1 0})^{5}$, 1-dimethylhydroxylsilyl-4-benzyldimethylsilyl1,3-butadiene $(\mathbf{1 7})^{6}, 3$-iodo-1-tetrahydropyranyloxy-2-propene $(\mathbf{1 8})^{7}$, ethyl 3-iodoacrylate ${ }^{8}$.

Tetraenephosphonate $\mathbf{1}$ was prepared from THP ether $\mathbf{2 0}$ according to the procedure described by Mori et al. ${ }^{9}$

Scheme 1. Synthesis of RK-397. Characterized compounds not appearing in the text are shown in bold italic type.

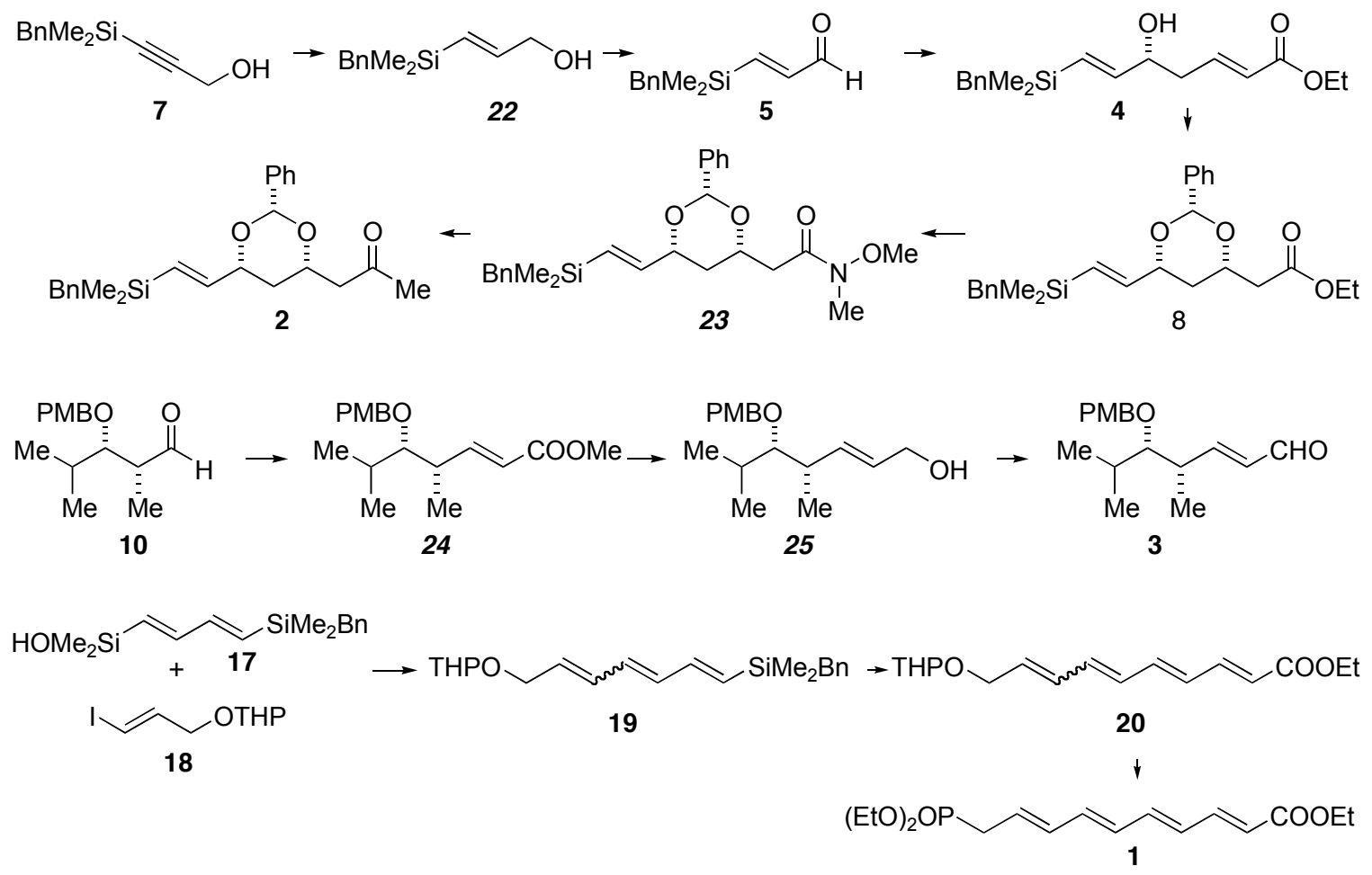


Scheme 2. Synthesis of RK-397 (continued).

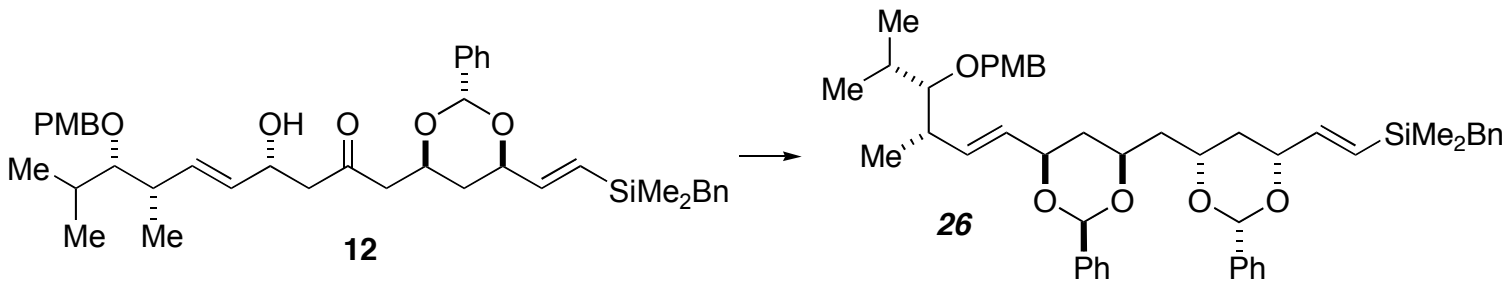<smiles>CC(C)/C=C/[C@@H](OC(C)(C)C)C(C)C</smiles><smiles>CCCCOC(C)C(C)[C@@H](C)/C=C/C1CC(CC2CC(CC=O)OC(c3ccccc3)O2)OC(c2ccccc2)O1</smiles>

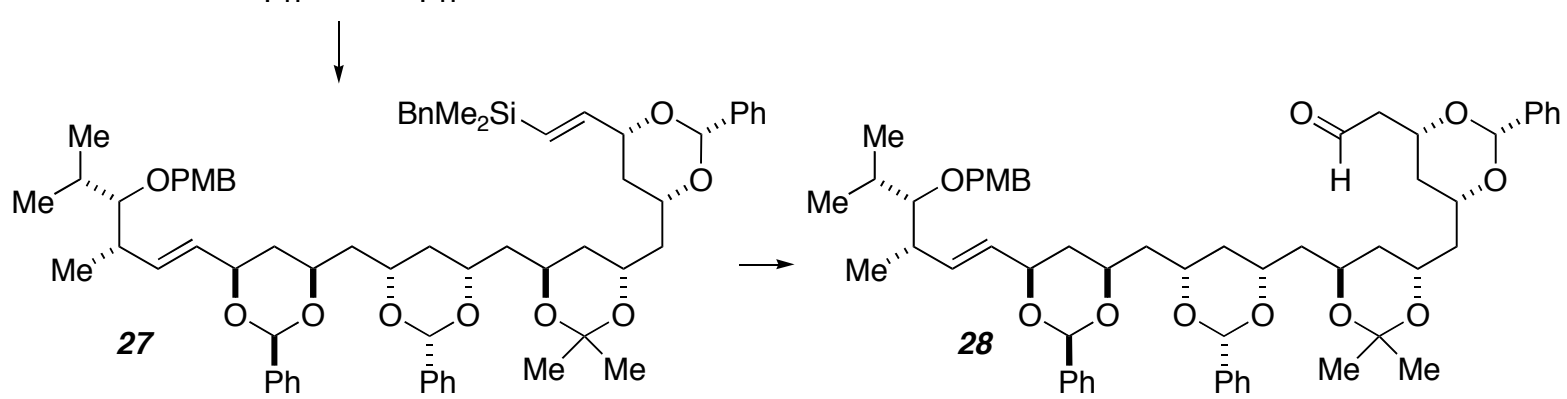

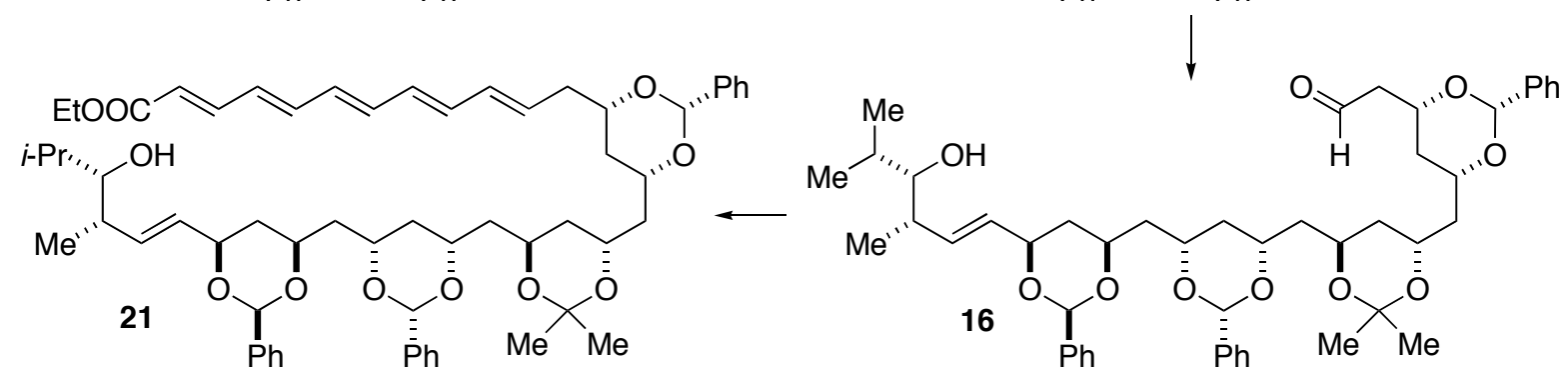

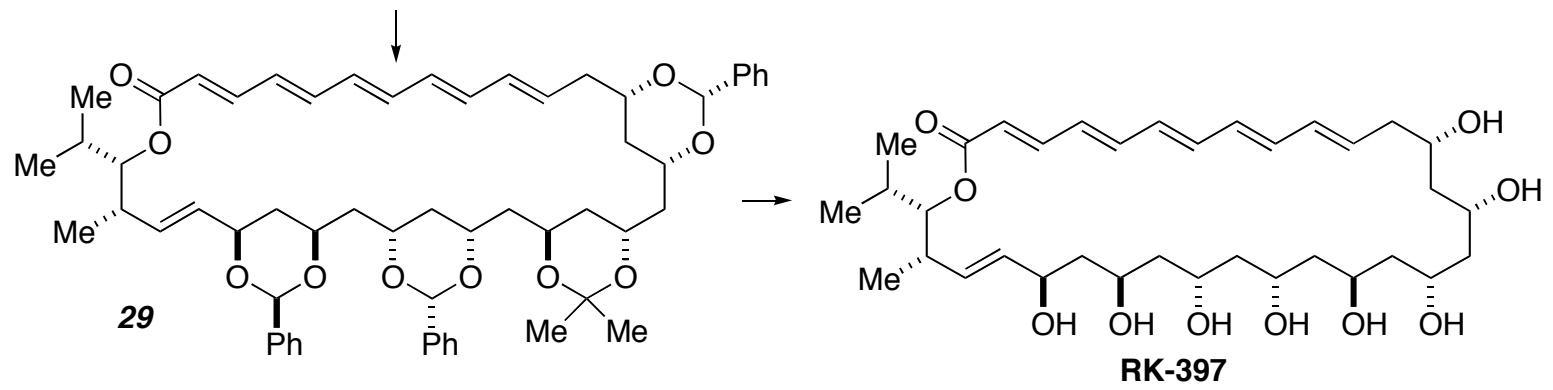




\section{Experimental Procedures}

\section{(E)-3-Benzyldimethylsilyl-2-propen-1-ol (22)}

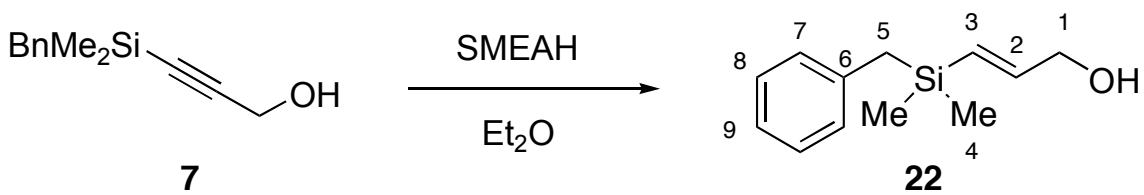

To a 500-mL 3-neck flask equipped with a 250-mL, pressure-equalized addition funnel, a septum (pierced with thermocouple probe), a gas inlet and a magnetic stir bar were placed sodium methoxyethoxyaluminium hydride (40.8 mL, $136 \mathrm{mmol}, 1.25$ equiv) and ether (100 mL). The solution was cooled to $0{ }^{\circ} \mathrm{C}$ using an ice bath. To the solution was added solution of 7 (22.3 $\mathrm{g}, 109 \mathrm{mmol}$ ) in $30 \mathrm{~mL}$ of ether dropwise over $1 \mathrm{~h}$ maintaining the internal temperature below 5 ${ }^{\circ} \mathrm{C}$. After the addition, the reaction mixture was warmed to $\mathrm{rt}$ and was stirred for $1 \mathrm{~h}$. The reaction mixture was cooled to $0{ }^{\circ} \mathrm{C}$ using an ice bath. The reaction mixture was carefully quenched with $1.8 \mathrm{M} \mathrm{H}_{2} \mathrm{SO}_{4}(200 \mathrm{~mL})$ maintaining the internal temperature below $10{ }^{\circ} \mathrm{C}$. The biphasic mixture was transferred to a 1-L separatory funnel, and the phases were separated. The aqueous layer was extracted with pentane $(2 \times 200 \mathrm{~mL})$. The combined organic layers were washed with saturated $\mathrm{NaHCO}_{3}(2 \times 100 \mathrm{~mL})$ and with brine $(100 \mathrm{~mL})$. The solution was dried over $\mathrm{Na}_{2} \mathrm{SO}_{4}$ and was concentrated under reduced pressure. The residue was distilled to afford $16.6 \mathrm{~g}$ (80.6 mmol, 74\%) of 22 as a clear, colorless oil.

\section{Data for 22:}

bp: $\quad 125^{\circ} \mathrm{C}(0.5 \mathrm{mmHg})$

${ }^{1} \mathrm{H}$ NMR: $\quad\left(500 \mathrm{MHz}, \mathrm{CDCl}_{3}\right)$

$7.22(\mathrm{t}, J=7.5,2 \mathrm{H}, 2 \times \mathrm{HC}(8)) ; 7.07$ (t, $J=7.5,1 \mathrm{H}, \mathrm{HC}(9)) ; 6.99$ (d, $J=7.0,2$ $\mathrm{H}, 2$ x HC(7)); $6.16(\mathrm{dt}, J=18.5,4.3,1 \mathrm{H}, \mathrm{HC}(2)) ; 5.92(\mathrm{dt}, J=18.5,1.8,1 \mathrm{H}$, $\mathrm{HC}(3)) ; 4.18\left(\mathrm{~m}, 2 \mathrm{H}, \mathrm{H}_{2} \mathrm{C}(1)\right), 2.14$ (s, $\left.2 \mathrm{H}, \mathrm{H}_{2} \mathrm{C}(5)\right)$; 1.36 (t, $\left.J=6.0,1 \mathrm{H}, \mathrm{HO}\right)$; $0.06\left(\mathrm{~s}, 6 \mathrm{H}, 2 \times \mathrm{H}_{3} \mathrm{C}(4)\right)$

${ }^{13} \mathrm{C} \mathrm{NMR}: \quad\left(126 \mathrm{MHz}, \mathrm{CDCl}_{3}\right)$

146.02 (C(2)); $139.83(\mathrm{C}(6)) ; 128.22$ (2 x C(8)); 128.11 (2 x C(7)); $127.30(\mathrm{C}(3))$; 
124.00 (C9)); $65.42(\mathrm{C}(1)) ; 25.37 \mathrm{C}(5)) ;-3.50(\mathrm{C} 4))$

IR: (neat)

3368 (br, s); 3081 (m); 3024 (m); 2955(s); 2917(s); 1618 (m); 1600 (m); 1492 (s); 1451(m); 1407 (w); 1248 (m); 1207 (m); 1154 (m); 1057 (m)

MS: $\quad(\mathrm{FI})$

$206\left(100, \mathrm{M}^{+}\right) ; 170$ (12); 156 (10); 126 (10); 115 (10); 114 (13); 60 (17)

TLC: $\quad R_{f} 0.28$ (hexane/EtOAc, $1 / 1$ ) [silica gel, $\mathrm{KMnO}_{4}$ ]

Analysis: $\quad \mathrm{C}_{12} \mathrm{H}_{18} \mathrm{OSi}(206.36)$

Calcd: $\quad$ C, $69.84 \%$; H, $8.39 \%$

Found: $\quad \mathrm{C}, 69.67 \% ; \quad \mathrm{H}, 8.54 \%$

\section{(E)-3-Dimethylsilyl-2-propenal (5)}

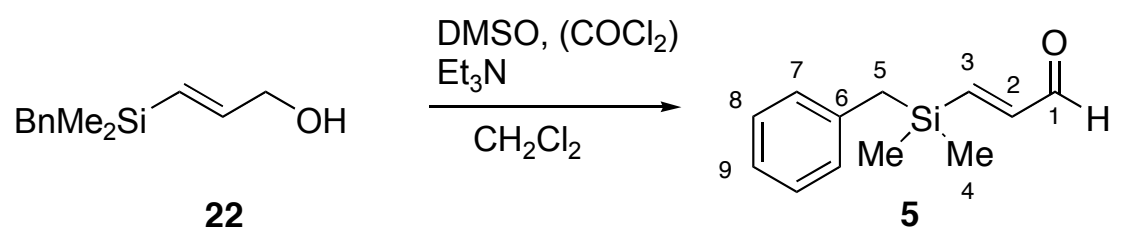

In a 500-mL, Schlenk flask were placed oxalyl chloride ( $10.2 \mathrm{~mL}, 117 \mathrm{mmol}, 1.5$ equiv) and dichloromethane $(200 \mathrm{~mL})$. The solution was cooled to $-70{ }^{\circ} \mathrm{C}$ (internal temperature) using an isopropanol/dry ice bath. To the solution was added dimethyl sulfoxide $(13.8 \mathrm{~mL}, 194 \mathrm{mmol}$, 2.5 equiv) dropwise maintaining internal temperature below $-50{ }^{\circ} \mathrm{C}$ over $20 \mathrm{~min}$. The reaction mixture was stirred for $10 \mathrm{~min}$. To the reaction mixture was added solution of $22(16.0 \mathrm{~g}, 77.7$ $\mathrm{mmol}$ ) in $50 \mathrm{~mL}$ of dichloromethane dropwise over 20 min maintaining internal temperature below $-50{ }^{\circ} \mathrm{C}$. The reaction mixture was stirred for $20 \mathrm{~min}$. To the reaction mixture was added triethylamine $(38.0 \mathrm{~mL}, 272 \mathrm{mmol}, 3.5$ equiv) dropwise over $30 \mathrm{~min}$ maintaining internal temperature below $-30{ }^{\circ} \mathrm{C}$. The reaction mixture was stirred for $30 \mathrm{~min}$ at $-70{ }^{\circ} \mathrm{C}$ and for $1 \mathrm{~h}$ after warming to $0{ }^{\circ} \mathrm{C}$. To the reaction mixture was added $100 \mathrm{~mL}$ of water and $100 \mathrm{~mL}$ of pentane. The biphasic solution was transferred to a 1-L separatory funnel. The organic layer was successively washed with $1 \mathrm{M} \mathrm{HCl}(100 \mathrm{~mL})$, water $(100 \mathrm{~mL})$, saturated $\mathrm{NaHCO}_{3}(100 \mathrm{~mL})$ and brine $(100 \mathrm{~mL})$. The solution was dried over $\mathrm{Na}_{2} \mathrm{SO}_{4}$ and concentrated under reduced 
pressure. The resulting oil was distilled under vacuum to afford $13.1 \mathrm{~g}(64.1 \mathrm{mmol}, 83 \%)$ of 5 as a clear, pale-yellow oil.

\section{Data for 5:}

bp: $\quad 108{ }^{\circ} \mathrm{C}(0.5 \mathrm{~mm} \mathrm{Hg})$

${ }^{1}$ H NMR: $\quad\left(500 \mathrm{MHz}, \mathrm{CDCl}_{3}\right)$

9.49 (d, $J=8.0,1 \mathrm{H}, \mathrm{HC}(1)) ; 7.23$ (t, $J=7.5,2 \mathrm{H}, 2 \times \mathrm{HC}(8)) ; 7.11$ (t, $J=7.0,1$ H, HC(9)); 6.99 (d, $J=7.0,2 \mathrm{H}, 2 \times \mathrm{HC}(7)) ;$ )); 7.14 (d, $J=19.0,1 \mathrm{H}, \mathrm{HC}(3))$; $6.48\left(\mathrm{dd}, J=18.5,7.5,1 \mathrm{H}, \mathrm{HC}(2) ; 2.23\right.$ (s, $\left.2 \mathrm{H}, \mathrm{H}_{2} \mathrm{C}(5)\right) ; 0.16$ (s, $6 \mathrm{H}, 2$ x $\left.\mathrm{H}_{3} \mathrm{C}(4)\right)$

${ }^{13} \mathrm{C} \mathrm{NMR}: \quad\left(126 \mathrm{MHz}, \mathrm{CDCl}_{3}\right)$

$194.53(\mathrm{C}(1)) ; 156.63(\mathrm{C}(3)) ; 144.87(\mathrm{C}(2)) ; 138.44(\mathrm{C}(6)) ; 128.38$ (2 x C(8)); 128.13 (2 x C(7)); 124.53 (C(9)); 24.90 (C(5)); -4.01 (2 x C(4))

IR: (neat)

3059 (w); 3024 (m); 2958(m); 2798(m); 1692 (s); 1599 (m); 1493 (m); 1452(m); 1407 (w); 1251 (m); 1206 (m); 1157 (m); 1085 (m)

MS: $\quad(\mathrm{FI})$

$204\left(100, \mathrm{M}^{+}\right)$

TLC: $\quad R_{f} 0.25$ (hexane/EtOAc, 5/1) [silica gel, $\mathrm{KMnO}_{4}$ ]

Analysis: $\quad \mathrm{C}_{12} \mathrm{H}_{16} \mathrm{OSi}(204.34)$

Calcd: $\quad$ C, $70.53 \% ; \quad \mathrm{H}, 7.89 \%$

Found: $\quad$ C, $70.25 \% ; \quad H, 8.04 \%$ 


\section{(5R)-Ethyl 7-benzyldimethylsilyl-5-hydroxy-hepta-2,6-dienoate (4)}

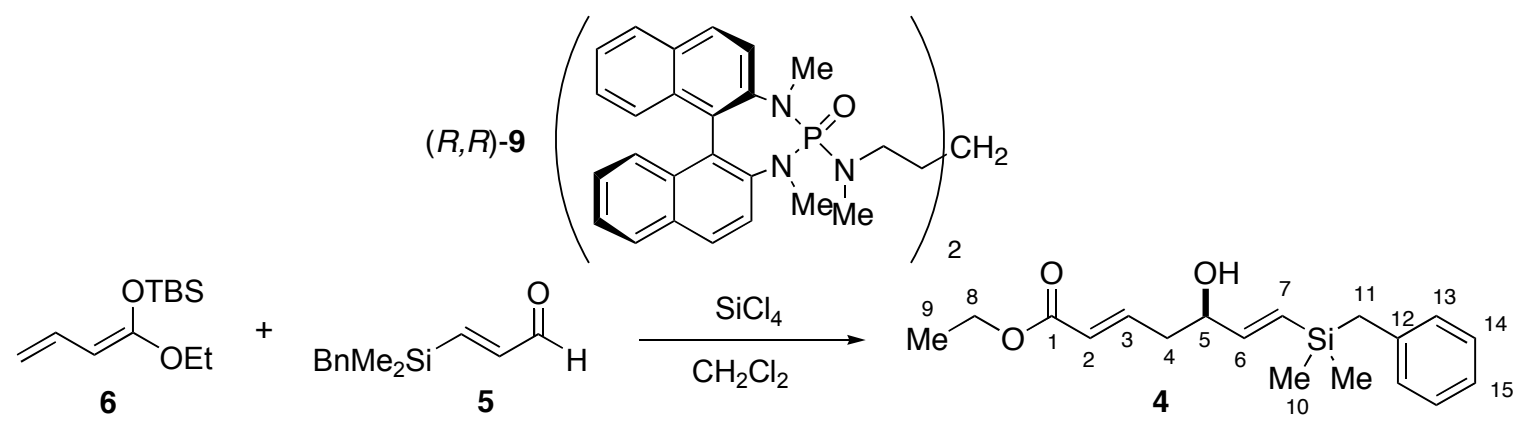

In a 100-mL Schlenk flask were placed phosphoramide $(R, R)-9(220 \mathrm{mg}, 0.26 \mathrm{mmol}$, 0.015 equiv), dichloromethane $(50 \mathrm{~mL})$, aldehyde $5(3.72 \mathrm{~g}, 18.2 \mathrm{mmol})$ and diisopropylethylamine $\left(0.65 \mathrm{~mL}, 3.64 \mathrm{mmol}, 0.20\right.$ equiv). The solution was cooled to $-70{ }^{\circ} \mathrm{C}$ (internal temperature measured by thermocouple) using an isopropanol/dry ice bath. To the reaction mixture was added silicon tetrachloride $(2.29 \mathrm{~mL}, 20.0 \mathrm{mmol}, 1.1$ equiv) followed by ketene acetal 6 (5.00 g, $21.8 \mathrm{mmol}, 1.2$ equiv) dropwise over $20 \mathrm{~min}$ maintaining the internal temperature below $-68{ }^{\circ} \mathrm{C}$. The reaction mixture was stirred at $-70{ }^{\circ} \mathrm{C}$ for $3 \mathrm{hrs}$. Then the reaction mixture was transferred via cannula into a vigorously stirring saturated aqueous $\mathrm{NaHCO}_{3}$ solution $(200 \mathrm{~mL})$ at $0{ }^{\circ} \mathrm{C}$ (bath temperature). The resulting mixture was stirred vigorously for $3 \mathrm{~h}$ at $\mathrm{rt}$. The solution was filtered through a layer of Celite, and the filtrate was transferred into a 500-mL separatory funnel. The aqueous layer was extracted with dichloromethane $(2 \times 100 \mathrm{~mL})$, and the combined organic layers were washed with brine (100 $\mathrm{mL}$ ). The organic solution was dried over $\mathrm{Na}_{2} \mathrm{SO}_{4}$ and concentrated under reduced pressure to give $8.7 \mathrm{~g}$ of a crude oil. The crude product was chromatographed (silica gel, hexane/EtOAc 3/1, $60 \mathrm{~mm})$ to afford $4.35 \mathrm{~g}(13.7 \mathrm{mmol}, 75 \%)$ of 4 as a clear, colorless oil.

\section{Data for 4:}

\section{${ }^{1}$ H NMR: $\quad\left(500 \mathrm{MHz}, \mathrm{CDCl}_{3}\right)$}

$7.20(\mathrm{t}, J=7.7,2 \mathrm{H}, 2 \times \mathrm{HC}(14)) ; 7.06$ (t, $J=7.3,1 \mathrm{H}, \mathrm{HC}(15)) ; 6.98$ (d, $J=7.1$, $2 \mathrm{H}, 2 \times \mathrm{HC}(13)) ; 6.94$ (dd, $J=15.6,7.5,1 \mathrm{H}, \mathrm{HC}(3))$; 6.01 (dd, $J=18.8,5.2,1$ $\mathrm{H}, \mathrm{HC}(6)) ; 5.90(\mathrm{dd}, J=15.8,1.5,1 \mathrm{H}, \mathrm{HC}(2) ; 5.87$ (dd, $J=18.9,1.2,1 \mathrm{H}$, $\mathrm{HC}(7)$ ); 4.22 (qd, $J=5.6,1.1,1 \mathrm{H}, \mathrm{HC}(5)) ; 4.19$ (q, $J=7.2,2 \mathrm{H}, \mathrm{H}_{2} \mathrm{C}(8)$ ); 2.34- 
2.46 (m, $\left.2 \mathrm{H}, \mathrm{H}_{2} \mathrm{C}(4)\right) ; 2.14$ (s, $\left.2 \mathrm{H}, \mathrm{H}_{2} \mathrm{C}(11)\right) ; 1.60$ (d, J = 4.5, $\left.1 \mathrm{H}, \mathrm{HO}\right) ; 1.28$ (t, $\left.J=7.1,3 \mathrm{H}, \mathrm{H}_{3} \mathrm{C}(9)\right) ; 0.06$ (s, $6 \mathrm{H}, 2 \times \mathrm{H}_{3} \mathrm{C}(10)$ )

${ }^{13} \mathrm{C} \mathrm{NMR}: \quad\left(126 \mathrm{MHz}, \mathrm{CDCl}_{3}\right)$

166.24 (C(1)); 148.18 (C(6)); 144.54 (C(3)); 139.58 (C(12)); 128.12 (2 x C(13)); 128.08 (C(7)); 128.03 (2 x C(14)); 123.97 (C(15)); 123.87 (C(2)); 72.74 (C(5)); $60.23(\mathrm{C}(8)) ; 39.54(\mathrm{C}(4)) ; 25.78(\mathrm{C}(11)) ; 14.16(\mathrm{C}(9)) ;-3.53$ (2 x C(10))

IR: (neat)

3437 (br, m); 3060 (w); 3024 (m); 2982 (m); 2956(m); 2898(m); 1720 (s); 1655

(m); $1619(\mathrm{w}) ; 1600(\mathrm{~m}) ; 1493(\mathrm{~m}) ; 1452(\mathrm{~m}) ; 1396(\mathrm{w}) ; 1369(\mathrm{~m}) ; 1316(\mathrm{~m})$; 1249 (m); 1207 (m); 1157 (m); 1096 (m); 1042 (m)

MS: $\quad(\mathrm{FI})$

$319\left(100, \mathrm{M}^{+}\right) ; 227(55)$

Opt. Rot.: $\quad[\alpha]_{\mathrm{D}}^{24}-5.79(\mathrm{c}=0.36, \mathrm{EtOH})$

TLC: $\quad R_{f} 0.08$ (hexane/EtOAc, 5/1) [silica gel, $\mathrm{KMnO}_{4}$ ]

SFC: $\quad$ (Chiralpak-AD, $3.0 \mathrm{~mL} / \mathrm{min}, 4.0 \% \mathrm{MeOH}, 125 \mathrm{bar}$ );

(5R)-4: $5.31 \min (98.1 \%)$; (5S)-4: $5.74 \min (1.9 \%)$

Analysis: $\quad \mathrm{C}_{18} \mathrm{H}_{26} \mathrm{O}_{3} \mathrm{Si}(318.48)$

Calcd: $\quad$ C, $67.88 \% ; \quad H, 8.23 \%$

Found: $\quad$ C, $67.59 \% ; \quad H, 8.41 \%$

(2'R, 4'S, 6'R)-Ethyl (2'-phenyl-6'-(2-benzyldimethylsilylethenyl)-[1,3]dioxan-4'-yl)-acetate (8)

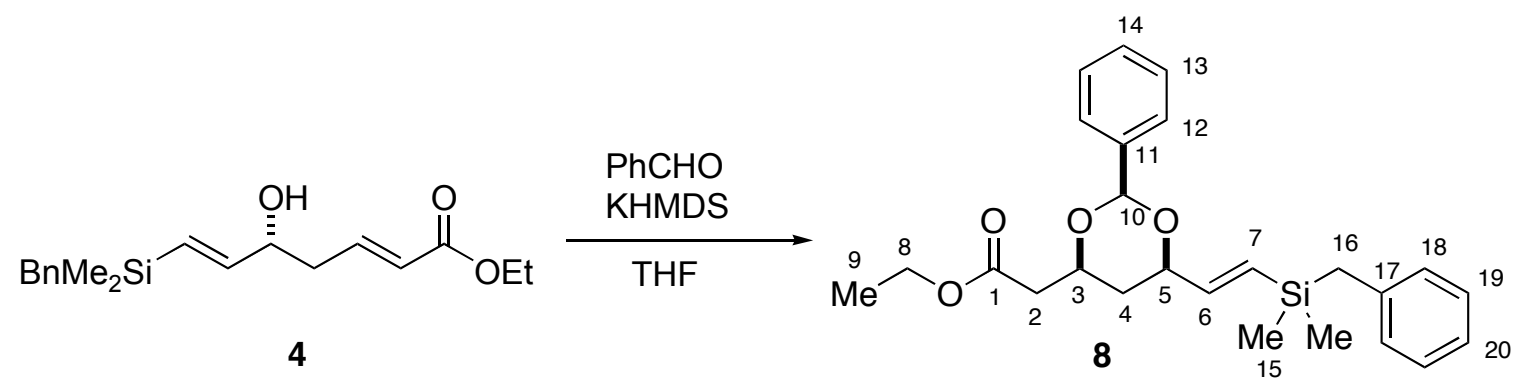

In a 500-mL Schlenk flask were placed 4 (3.41 g, $10.7 \mathrm{mmol})$, benzaldehyde $(1.23 \mathrm{~mL}$, 
$11.8 \mathrm{mmol}, 1.1$ equiv) and THF $(100 \mathrm{~mL})$. The solution was cooled to $0{ }^{\circ} \mathrm{C}$ (internal temperature monitored by thermocouple) using ice bath. To the solution was added 0.5 M KHMDS in THF $(2.13 \mathrm{~mL}, 1.07 \mathrm{mmol}, 0.1$ equiv) in one portion. The reaction mixture turned dark yellow and the internal temperature rose to $5^{\circ} \mathrm{C}$. The reaction mixture was stirred for $20 \mathrm{~min}$ at $0{ }^{\circ} \mathrm{C}$. To the reaction mixture were added benzaldehyde $(1.23 \mathrm{~mL}, 11.8 \mathrm{mmol}, 1.1$ equiv) and $0.5 \mathrm{M}$ KHMDS in THF $\left(2.13 \mathrm{~mL}, 1.07 \mathrm{mmol}, 0.1\right.$ equiv). The reaction mixture was stirred for $20 \mathrm{~min}$ at $0^{\circ} \mathrm{C}$. The addition and stirring sequence was repeated one more time before the reaction mixture was quenched with cold $\left(0{ }^{\circ} \mathrm{C}\right.$, bath temperature $) \mathrm{pH} 7$ phosphate buffer $(100 \mathrm{~mL})$. The biphasic mixture was poured into a 500-mL separatory funnel and was extracted with pentane $(200 \mathrm{~mL})$. The organic layer was washed with brine $(100 \mathrm{~mL})$, dried over $\mathrm{Na}_{2} \mathrm{SO}_{4}$ and concentrated under reduced pressure. Most of the remaining benzaldehyde was removed under vacuum with stirring. The resulting crude oil was chromatographed (silica gel, hexanes/EtOAc 9/1, $60 \mathrm{~mm}$ ) to afford $3.38 \mathrm{~g}(7.96 \mathrm{mmol}, 74 \%)$ of $\mathbf{8}$ as a pale-yellow oil.

\section{Data for 8:}

${ }^{1} \mathrm{H} \mathrm{NMR}: \quad\left(500 \mathrm{MHz}, \mathrm{CDCl}_{3}\right)$

$7.52(\mathrm{~d}, J=6.7,2 \mathrm{H}, 2 \times \mathrm{HC}(12)) ; 7.33-7.38$ (m, $3 \mathrm{H}, \mathrm{HC}(14), 2 \times \mathrm{HC}(13))$;

$7.20(\mathrm{t}, J=7.6,2 \mathrm{H}, 2 \times \mathrm{HC}(19)) ; 7.07$ (t, $J=7.4,1 \mathrm{H}, \mathrm{HC}(20)) ; 6.98$ (d, $J=7.2$,

$2 \mathrm{H}, 2 \times \mathrm{HC}(18)$ ); 6.07 (dd, $J=19.0,4.6,1 \mathrm{H}, \mathrm{HC}(6)) ; 5.94(\mathrm{dd}, \mathrm{J}=19.1,1.2,1$

H, HC(7); 5.62 (s, 1 H, HC(10));4.32 - 4.40 (m, 2 H, HC(3), HC(5)); 4.18 (q, J = 7.2, $\left.2 \mathrm{H}, \mathrm{H}_{2} \mathrm{C}(8)\right) ; 2.64\left(\mathrm{ABX}, J_{\mathrm{AB}}=5.7,2 \mathrm{H}, \mathrm{H}_{2} \mathrm{C}(2)\right) ; 2.13$ (s, $\left.2 \mathrm{H}, \mathrm{H}_{2} \mathrm{C}(16)\right)$; $1.78\left(\mathrm{dt}, J=13.0,7.3,1 \mathrm{H}, \mathrm{H}_{\mathrm{eq}} \mathrm{C}(4)\right) ; 1.53\left(\mathrm{q}, J=11.4,1 \mathrm{H}, \mathrm{H}_{\mathrm{ax}} \mathrm{C}(4)\right) ; 1.28(\mathrm{t}, J=$ 7.1, $\left.3 \mathrm{H}, \mathrm{H}_{3} \mathrm{C}(9)\right) ; 0.05$ (s, $6 \mathrm{H}, 2 \times \mathrm{H}_{3} \mathrm{C}(15)$ )

${ }^{13} \mathrm{C} \mathrm{NMR}: \quad\left(126 \mathrm{MHz}, \mathrm{CDCl}_{3}\right)$

170.61 (C(1)); 145.69 (C(6)); 139.62 (C(17)); 138.19 (C(11)); 128.64 (C(14)); 128.14 (C7)); 128.08 (2 x C(13)); 128.05 (2 x (C(18)); 128.00 (2 x C(19)); 126.10 (C(12)); 123.91 (C(20)); 100.42 (C(10)); 78.22 (C(5)); 72.97 (C(3)); 60.50 (C(8)); $40.75(\mathrm{C}(2)) ; 36.00(\mathrm{C}(4)) ; 25.71(\mathrm{C}(16)) ; 14.06(\mathrm{C}(9)) ;-3.71,-3.66(2 \times \mathrm{C}(15))$

IR: (neat)

3061 (w); 3024 (m); 2981 (m); 2957(m); 2897(m); 1736 (s); 1623 (m); 1600 (m); 1493 (m); 1452(m); 1401 (m); 1373 (m); $1339(\mathrm{~m}) ; 1314(\mathrm{~m}) ; 1250(\mathrm{~m}) ; 1206$ 
(m); $1158(\mathrm{~m}) ; 1112(\mathrm{~m}) ; 1097(\mathrm{~m}) ; 1056(\mathrm{~m})$

MS: $\quad(\mathrm{FI})$

$425\left(52, \mathrm{M}^{+}\right) ; 424$ (27); 423 (31); 422 (18); 351 (19); 335 (88); 333 (47); 317

(43); 316 (35); 291 (38); 290 (97); 243 (38); 220 (92); 219 (29); 190 (67); 128 (27); 106 (100); 91 (45)

Opt. Rot.: $\quad[\alpha]^{24}+15.3(\mathrm{c}=0.57, \mathrm{EtOH})$

TLC: $\quad R_{f} 0.33$ (pentane /ether 9/1) [silica gel, $\mathrm{KMnO}_{4}$ ]

Analysis: $\quad$ Calc for $\mathrm{C}_{25} \mathrm{H}_{32} \mathrm{O}_{4} \mathrm{Si}$ (424.60)
Calcd:
C, 70.72\%;
$\mathrm{H}, 7.60 \%$
Found:
C, $70.73 \%$;
$\mathrm{H}, 7.52 \%$

(2'R, 4'S, 6'R)-N,O-Dimethyl (2'-phenyl-6'-(2-benzyldimethylsilylethenyl)-[1,3]dioxan-4'yl)-acetamide (23)
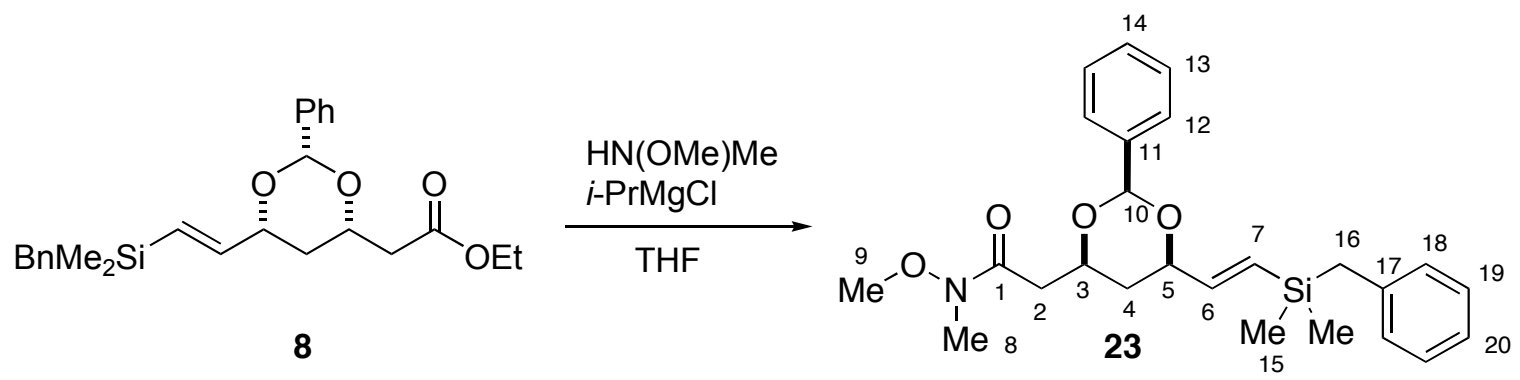

In a 50-mL Schlenk flask were placed $8(2.20 \mathrm{~g}, 5.18 \mathrm{mmol}), \quad \mathrm{N}, \mathrm{O}-$ dimethylhydroxylamine hydrochloride $(758 \mathrm{mg}, 7.77 \mathrm{mmol}, 1.5$ equiv) and THF (11 mL). The reaction mixture was cooled to $-20{ }^{\circ} \mathrm{C}$ (internal temperature monitored by thermocouple) using $\mathrm{NaCl} /$ ice bath. To the reaction mixture was added $2 \mathrm{M}$ solution of isopropylmagnesium chloride in THF (7.77 mL, 15.5, 3 equiv) dropwise over 30 min maintaining internal temperature below $10{ }^{\circ} \mathrm{C}$. The reaction mixture was stirred at $-20{ }^{\circ} \mathrm{C}$ for $20 \mathrm{~min}$ and was quenched with saturated aqueous $\mathrm{NH}_{4} \mathrm{Cl}$ solution $(20 \mathrm{~mL})$. The reaction mixture was poured into a $250-\mathrm{mL}$ separatory funnel and extracted with pentane $(100 \mathrm{~mL})$. The organic phase was washed with brine $(25 \mathrm{~mL})$, dried over $\mathrm{Na}_{2} \mathrm{SO}_{4}$ and concentrated. The resulting oil was chromatographed (silica gel, hexane/EtOAc 2/1, $50 \mathrm{~mm})$ to afford $1.98 \mathrm{~g}$ (4.50 mmol, 87\%) of 23 as a clear, colorless oil. 


\section{Data for 23:}

${ }^{1} \mathrm{H} \mathrm{NMR}: \quad\left(500 \mathrm{MHz}, \mathrm{CDCl}_{3}\right)$

$7.53(\mathrm{dd}, J=8.0,1.4,2 \mathrm{H}, 2$ x $\mathrm{HC}(12)) ; 7.32-7.38(\mathrm{~m}, 3 \mathrm{H}, \mathrm{HC}(14), 2 \times$ $\mathrm{HC}(13)) ; 7.21$ (t, $J=7.7,2 \mathrm{H}, 2$ x HC(19)); 7.07 (t, $J=7.5,1 \mathrm{H}, \mathrm{HC}(20)) ; 6.99$ (d, $J=7.1,2 \mathrm{H}, 2 \times \mathrm{HC}(18)) ; 6.09$ (dd, $J=19.1,4.7,1 \mathrm{H}, \mathrm{HC}(6)) ; 5.95$ (dd, $J=$ 18.9, 1.3, $1 \mathrm{H}, \mathrm{HC}(7))$; 5.64 (s, $1 \mathrm{H}, \mathrm{HC}(10))$; 4.38 - 4.46 (m, $2 \mathrm{H}, \mathrm{HC}(3)$, $\mathrm{HC}(5)) ; 3.68\left(\mathrm{~s}, 3 \mathrm{H}, \mathrm{H}_{3} \mathrm{C}(9)\right) ; 3.20\left(\mathrm{~s}, 3 \mathrm{H}, \mathrm{H}_{3} \mathrm{C}(8)\right) ; 2.79\left(\mathrm{ABX}, J_{\mathrm{AB}}=15.6,2 \mathrm{H}\right.$, $\left.\mathrm{H}_{2} \mathrm{C}(2)\right) ; 2.14$ (s, $\left.2 \mathrm{H}, \mathrm{H}_{2} \mathrm{C}(16)\right) ; 1.90$ (dt, $\left.J=13.2,2.4,1 \mathrm{H}, \mathrm{H}_{\mathrm{eq}} \mathrm{C}(4)\right) ; 1.52$ (q, $J=$ 12.4, $\left.1 \mathrm{H}, \mathrm{H}_{\mathrm{ax}} \mathrm{C}(4)\right)$; 0.05, 0.04 (s, $\left.6 \mathrm{H}, 2 \times \mathrm{H}_{3} \mathrm{C}(15)\right)$

${ }^{13} \mathrm{C} \mathrm{NMR}: \quad\left(126 \mathrm{MHz}, \mathrm{CDCl}_{3}\right)$

$171.24(\mathrm{C}(1)) ; 145.90(\mathrm{C}(6)) ; 139.78$ (C(17)); 138.43 (C(11)); 128.73 (C(7)); 128.24 ( 2 x C(18)); 128.17 ( 2 x C(13), 2 x C(19)); 128.09 (C(14)); 126.24 (2 x $\mathrm{C}(12)) ; 123.97(\mathrm{C}(20)) ; 100.70(\mathrm{C}(10)) ; 78.46(\mathrm{C}(5)) ; 73.40(\mathrm{C}(3)) ; 61.37(\mathrm{C}(9))$; $38.08(\mathrm{C}(2)) ; 36.53(\mathrm{C}(4)) ; 31.93(\mathrm{C}(8)) ; 25.81(\mathrm{C}(16)) ;-3.63,-3.60(2 \times \mathrm{C}(15))$

IR: (neat)

3060 (w); 3024 (m); 2998 (m); 2956(m); $2916(\mathrm{~m}) ; 2894(\mathrm{~m}) ; 1664(\mathrm{~s}) ; 1623$ (s); 1600 (m); 1493 (m); 1452(m); 1413 (m); 1388 (m); 1336 (m); 1313 (w); 1248(m); 1208 (m); 1176 (m); 1128 (m); 1097 (m); 1056 (m); 1027 (m)

MS: $\quad(\mathrm{FI})$

440 (3, M+); 437 (14); 436 (43); 434 (11); 423 (22); 422 (67); 420 (13); 409 (31); 408 (100); $381(23) ; 380(77) ; 366(28)$

Opt. Rot.: $\quad[\alpha]_{D}^{24}+12.5(\mathrm{c}=0.50, \mathrm{EtOH})$

TLC: $\quad R_{f} 0.08$ (hexane/EtOAc 2/1) [silica gel, $\mathrm{KMnO}_{4}$ ]

Analysis: $\quad$ Calc for $\mathrm{C}_{25} \mathrm{H}_{33} \mathrm{NO}_{4} \mathrm{Si}$ (439.62)

Calcd: $\quad$ C, $68.30 \% ; \quad H, 7.57 \% ; \quad$ N, $3.19 \%$

Found: $\quad$ C, 68.28\%; H, 7.61\%; N, 3.29\% 
(2'R, 4'S, 6'R)-3-(2'-Phenyl-6'-(2-benzyldimethylsilylethenyl)-[1,3]dioxan-4'-yl)-propan-2one (2)

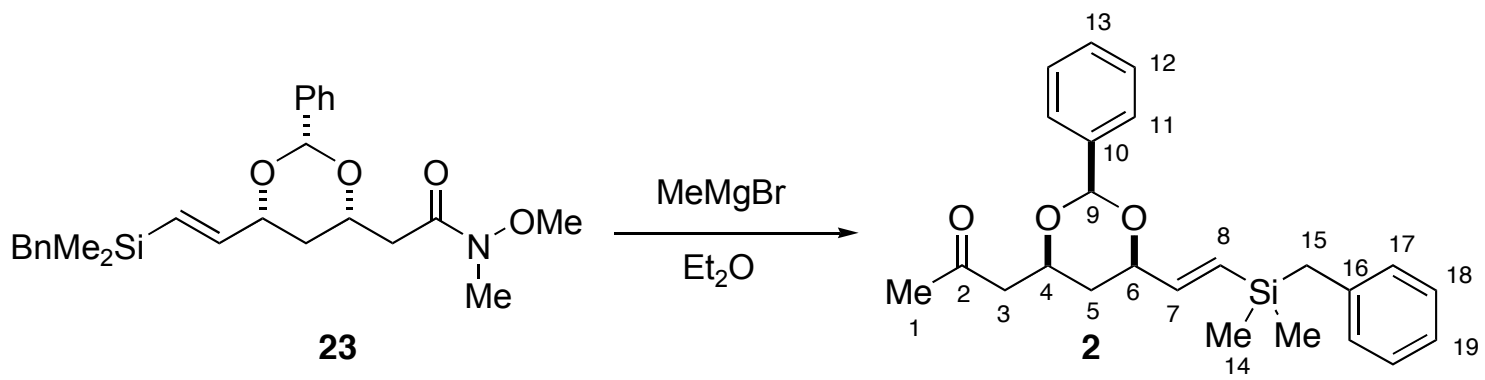

In a 100-mL Schlenk flask were placed $23(1.49 \mathrm{~g}, 3.39 \mathrm{mmol})$ and ether $(20 \mathrm{~mL})$. The reaction mixture was cooled to $0{ }^{\circ} \mathrm{C}$ in an ice bath. To the solution was added a $3 \mathrm{M}$ solution of methylmagnesium bromide in ether $(1.69 \mathrm{~mL}, 5.08 \mathrm{mmol}, 1.5$ equiv) dropwise over $1 \mathrm{~min}$. The reaction mixture was allowed to warm to $\mathrm{rt}$ and was stirred for $10 \mathrm{~h}$. The reaction was quenched with saturated aqueous $\mathrm{NH}_{4} \mathrm{Cl}$ solution $(10 \mathrm{~mL})$ and was poured into a $125-\mathrm{mL}$ separatory funnel, and the biphasic mixture was extracted with pentane $(80 \mathrm{~mL})$. The organic phase was washed with brine $(20 \mathrm{~mL})$, dried over $\mathrm{Na}_{2} \mathrm{SO}_{4}$ and concentrated. The resulting oil was chromatographed (silica gel, hexanes/EtOAc 3/1, $50 \mathrm{~mm}$ ) to afford $1.20 \mathrm{~g}(3.04 \mathrm{mmol}, 90 \%)$ of 2 as a clear, colorless oil.

Data for 2:

${ }^{1}$ H NMR: $\quad\left(500 \mathrm{MHz}, \mathrm{CDCl}_{3}\right)$

$7.51(\mathrm{dd}, J=7.8,1.5,2 \mathrm{H}, 2 \times \mathrm{HC}(11)) ; 7.32-7.38$ (m, $3 \mathrm{H}, \mathrm{HC}(13), 2 \times$ $\mathrm{HC}(12)) ; 7.20$ (t, $J=7.7,2$ H, 2 x HC(18)); 7.07 (t, $J=7.3,1 \mathrm{H}, \mathrm{HC}(19)) ; 6.99$ $(\mathrm{d}, J=7.3,2 \mathrm{H}, 2 \times \mathrm{HC}(17)) ; 6.07(\mathrm{dd}, J=18.9,4.5,1 \mathrm{H}, \mathrm{HC}(7)) ; 5.94(\mathrm{dd}, J=$ 18.8, 12, 1 H, HC(8)); 5.61 (s, 1 H, HC(9)); 4.32 - 4.40 (m, 2 H, HC(4), HC(6)); $2.75\left(\mathrm{ABX}, J_{\mathrm{AB}}=16.4, J_{\mathrm{AX}}=7.0, J_{\mathrm{BX}}=5.7,2 \mathrm{H}, \mathrm{H}_{2} \mathrm{C}(3)\right) ; 2.22\left(\mathrm{~s}, 3 \mathrm{H}, \mathrm{H}_{3} \mathrm{C}(1)\right)$; 2.13 (s, $\left.2 \mathrm{H}, \mathrm{H}_{2} \mathrm{C}(15)\right) ; 1.78$ (dt, J = 13.1, 2.5, $\left.1 \mathrm{H}, \mathrm{H}_{\mathrm{eq}} \mathrm{C}(5)\right) ; 1.47$ (dt, $J=13.0$, 11.3, $\left.1 \mathrm{H}, \mathrm{H}_{\mathrm{ax}} \mathrm{C}(5)\right)$; 0.05, 0.04 (s, $6 \mathrm{H}, 2 \times \mathrm{H}_{3} \mathrm{C}(14)$ )

${ }^{13} \mathrm{C}$ NMR: $\quad\left(126 \mathrm{MHz}, \mathrm{CDCl}_{3}\right)$

$206.25(\mathrm{C}(2)) ; 145.67(\mathrm{C}(8)) ; 139.59(\mathrm{C}(16)) ; 138.19(\mathrm{C}(10)) ; 128.63(\mathrm{C}(13))$; 
$128.18(\mathrm{C}(7)) ; 128.11(2 \times \mathrm{C}(17)) ; 128.05(2 \times \mathrm{C}(12)) ; 127.97(2 \times \mathrm{C}(18)) ; 126.02$ (2 x C(11)); 123.89 (C(19)); 100.39 (C(9)); 78.38 (C(6)); 76.26 (C(4)); 49.15 $(\mathrm{C}(3)) ; 36.15(\mathrm{C}(5)) ; 31.00(\mathrm{C}(1)) ; 25.68(\mathrm{C}(15)) ;-3.72,-3.68(2 \times \mathrm{C}(14))$

IR: (neat)

3060 (w); 3025 (m); 2999 (m); 2958(m); 2916 (m); 1716 (s); 1623 (m); 1600 (m); 1494 (m); 1452(m); 1403 (m); 1337 (m); 1316 (w); 1248(m); 1208 (m); 1159 (m); 1133 (m); 1099 (m); 1055 (m); 1027 (m)

MS: $\quad(\mathrm{FI})$

$394\left(100, \mathrm{M}^{+}\right) ; 306$ (15); 305 (50); 304 (21); 303 (80); 270 (17); 204 (11); 161

(17); 152 (12); 115 (12); 114 13), 113 (14); 106 (11)

Opt. Rot.: $\quad[\alpha]_{D}^{24}+4.9(\mathrm{c}=0.85, \mathrm{EtOH})$

TLC: $\quad R_{f} 0.28$ (hexanes/EtOAc 3/1) [silica gel, $\mathrm{KMnO}_{4}$ ]

Analysis: $\quad$ Calc for $\mathrm{C}_{24} \mathrm{H}_{30} \mathrm{O}_{3} \mathrm{Si}$ (394.58)

Calcd: $\quad$ C, $73.05 \%$; $\mathrm{H}, 7.66 \%$

Found: $\quad$ C, $72.96 \% ; \quad H, 7.87 \%$

\section{(4S, 5S)-Methyl 4,6-dimethyl-5-(4-methoxyphenylmethoxy)-2-heptenoate (24)}

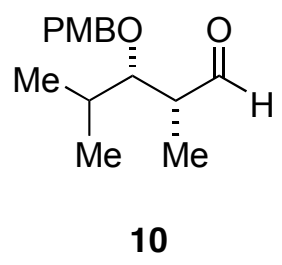

In a 250-mL flask were placed $\mathbf{1 0}(4.43 \mathrm{~g}, 17.7 \mathrm{mmol})$, methyl triphenylphosphanylideneacetate $(8.88 \mathrm{~g}, 26.6,1.5$ equiv) and acetonitrile $(100 \mathrm{~mL})$. The flask was fitted with a reflux condenser and was immersed into an oil bath. The mixture was heated to $90{ }^{\circ} \mathrm{C}$ (bath temperature) for $24 \mathrm{hrs}$. The reaction mixture was cooled to $\mathrm{rt}$ and was placed under reduced pressure $(20 \mathrm{mmHg})$ to remove most of the acetonitrile. The resulting solid/oil mixture was chromatographed (silica gel, hexanes/EtOAc 9/1, $60 \mathrm{~mm}$ ) to afford $4.86 \mathrm{~g} \mathrm{(15.9} \mathrm{mmol,} \mathrm{90 \% )}$ 
of $\mathbf{2 4}$ as a clear, colorless oil.

\section{Data for 24:}

${ }^{1} \mathrm{H} \mathrm{NMR}: \quad\left(500 \mathrm{MHz}, \mathrm{CDCl}_{3}\right)$

7.27 (d, $J=8.5,2$ H, 2 x HC(12)); $7.02(\mathrm{dd}, J=15.9,8.1,1 \mathrm{H}, \mathrm{HC}(3)) ; 6.88$ (d, $J$ $=8.6,2 \mathrm{H}, 2 \times \mathrm{HC}(13)) ; 5.86(\mathrm{dd}, J=15.6,1.2,1 \mathrm{H}, \mathrm{HC}(2)) ; 4.48(\mathrm{~s}, 2 \mathrm{H}$, $\left.\mathrm{H}_{2} \mathrm{C}(10)\right) ; 3.84$ (s, $\left.3 \mathrm{H}, \mathrm{H}_{3} \mathrm{C}(15)\right) ; 3.74$ (s, $\left.3 \mathrm{H}, \mathrm{H}_{3} \mathrm{C}(8)\right) ; 3.08$ (t, $J=5.6,1 \mathrm{H}$, $\mathrm{HC}(5)) ; 2.62$ (sextd, $J=6.6,1.0,1 \mathrm{H}, \mathrm{HC}(4)) ; 1.82$ (sext, $J=6.8,1 \mathrm{H}, \mathrm{HC}(6)$ ); $1.12\left(\mathrm{~d}, J=6.6,3 \mathrm{H}, \mathrm{H}_{3} \mathrm{C}(9)\right) ; 0.96$ (d, $\left.J=6.9,3 \mathrm{H}, \mathrm{H}_{3} \mathrm{C}(7)\right) ; 0.95$ (d, $J=6.6,3 \mathrm{H}$, $\left.\mathrm{H}_{3} \mathrm{C}\left(7^{\prime}\right)\right)$

${ }^{13} \mathrm{C} \mathrm{NMR}: \quad\left(126 \mathrm{MHz}, \mathrm{CDCl}_{3}\right)$

167.11 (C(1)); 159.08 (C(14)); 152.69 (C(3)); 130.72 (C(11)); 129.29 (C(12)); $119.93(\mathrm{C}(2)) ; 113.64$ (C(13)); 87.35 (C(5)); 74.77 (C(10)); 55.14 (C(15)); 51.36 (C(8)); 39.70 (C(4)); 31.19 (C(6)); 20.17 (C(7)); 17.48 (C(7’)); 14.58 (C(9))

IR: (neat)

2961 (m); 2909(m); 2874 (m); 2838 (m); 1723 (s); 1655 (m); 1613 (m); 1586 (m); $1514(\mathrm{~m}) ; 1464(\mathrm{~m}) ; 1436(\mathrm{~m}) ; 1383(\mathrm{w}) ; 1364(\mathrm{w}) ; 1336(\mathrm{~m}) ; 1301(\mathrm{~m}) ; 1249$ (s); 1194 (m); 1175 (m); 1137 (w); 1110 (m); 1063 (m); 1036 (m); 1012 (m)

MS: $\quad(\mathrm{FI})$

307 (20); $306\left(100, \mathrm{M}^{+}\right) ; 276(15)$

Opt. Rot.: $\quad[\alpha]_{\mathrm{D}}^{24}+5.0(\mathrm{c}=0.90, \mathrm{EtOH})$

TLC: $\quad R_{f} 0.18$ (hexanes/EtOAc 9/1) [silica gel, $\mathrm{KMnO}_{4}$ ]

Analysis: $\quad$ Calc for $\mathrm{C}_{14} \mathrm{H}_{26} \mathrm{O}_{4}(306.40)$

Calcd: $\quad$ C, $70.56 \% ; \quad H, 8.55 \%$

Found: $\quad$ C, $70.57 \%$; $\quad$ H, $8.52 \%$ 


\section{(4S, 5S)-4,6-Dimethyl-5-(4-methoxy-phenylmethoxy)-2-hepten-1-ol (25)}

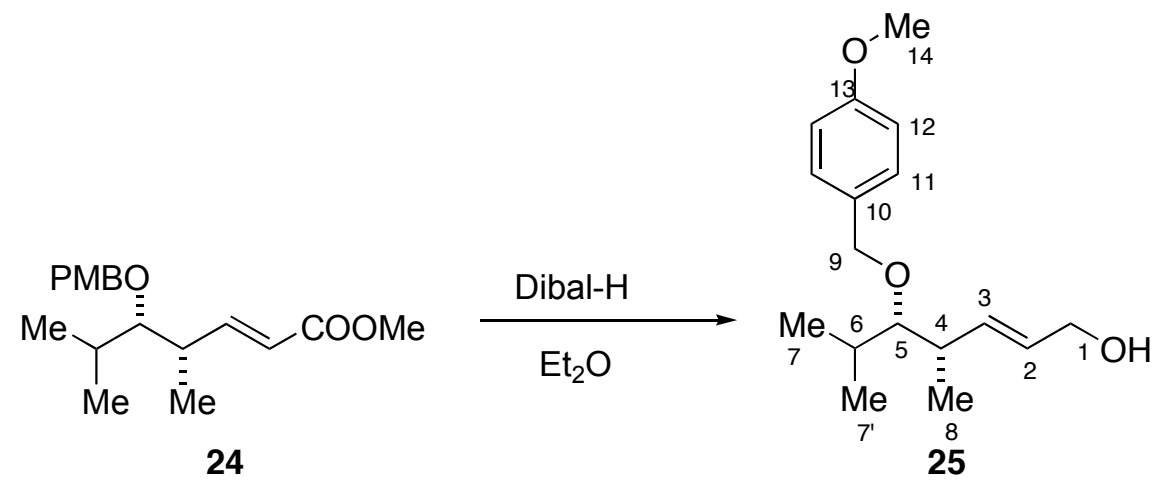

In a $250-\mathrm{mL}$, three-neck flask equipped with a 50-mL pressure-equalizing addition funnel, a septum pierced with thermocouple temperature probe and a gas inlet were placed 24 (4.33 g, $14.1 \mathrm{mmol})$ and ether $(90 \mathrm{~mL})$. The solution was cooled to $-74{ }^{\circ} \mathrm{C}$ (internal temperature) using isopropanol/dry ice bath. The addition funnel was charged with $1 \mathrm{M}$ solution of diisobutylaluminum hydride in hexanes $(42.3 \mathrm{~mL}, 42.3 \mathrm{mmol}, 3$ equiv). The solution of diisobutylaluminum hydride was added dropwise over 30 min maintaining temperature below $-65{ }^{\circ} \mathrm{C}$. The reaction mixture was stirred for $30 \mathrm{~min}$ at $-74{ }^{\circ} \mathrm{C}$, allowed to warm to $0{ }^{\circ} \mathrm{C}$ and was stirred for $1 \mathrm{~h}$ at $0{ }^{\circ} \mathrm{C}$. At this temperature, the reaction mixture was carefully quenched with $10 \% \mathrm{v} / \mathrm{v}$ aqueous acetic acid $(100 \mathrm{~mL})$ maintaining internal temperature below $10{ }^{\circ} \mathrm{C}$. The resulting clear solution was poured into a 500-mL separatory funnel. The aqueous layer was extracted with ether $(2 \times 100 \mathrm{~mL})$, and the combined organic layers were washed with water (50 $\mathrm{mL})$, saturated aqueous $\mathrm{NaHCO}_{3}$ solution $(2 \times 50 \mathrm{~mL})$ and brine $(50 \mathrm{~mL})$. The extracts were dried over $\mathrm{Na}_{2} \mathrm{SO}_{4}$ and concentrated under reduced pressure. The resulting clear oil was chromatographed (silica gel, hexanes/EtOAc 2/1, $60 \mathrm{~mm}$ ) to afford 25 (3.80 g, $13.6 \mathrm{mmol}, 97 \%)$ as clear colorless oil.

\section{Data for 25:}

${ }^{1} \mathrm{H}$ NMR: $\quad\left(500 \mathrm{MHz}, \mathrm{CDCl}_{3}\right)$

$7.28(\mathrm{~d}, J=8.5,2 \mathrm{H}, 2 \times \mathrm{HC}(11)) ; 6.87(\mathrm{~d}, J=8.6,2 \mathrm{H}, 2 \times \mathrm{HC}(12)) ; 5.64-5.68$ $(\mathrm{m}, 2 \mathrm{H}, \mathrm{HC}(2), \mathrm{HC}(3)) ; 4.50$ (s, $\left.2 \mathrm{H}, \mathrm{H}_{2} \mathrm{C}(9)\right) ; 4.09$ (td, $J=4.9,2.2,2 \mathrm{H}$, $\left.\mathrm{H}_{2} \mathrm{C}(1)\right) ; 3.80$ (s, $\left.3 \mathrm{H}, \mathrm{H}_{3} \mathrm{C}(14)\right) ; 2.98$ (t, $J=5.8,1 \mathrm{H}, \mathrm{HC}(5)$ ); 2.46 (sext, $J=6.5$, 
$1 \mathrm{H}, \mathrm{HC}(4)) ; 1.84$ (sext, $J=6.7,1 \mathrm{H}, \mathrm{HC}(6)) ; 1.23$ (t, $J=7.5,1 \mathrm{H}, \mathrm{HO}) ; 1.07$ (d, $J$ $\left.=6.8,3 \mathrm{H}, \mathrm{H}_{3} \mathrm{C}(8)\right) ; 0.96\left(\mathrm{~d}, J=6.8,3 \mathrm{H}, \mathrm{H}_{3} \mathrm{C}(7)\right) ; 0.95\left(\mathrm{~d}, J=6.8,3 \mathrm{H}, \mathrm{H}_{3} \mathrm{C}\left(7^{\prime}\right)\right)$

${ }^{13} \mathrm{C} \mathrm{NMR}: \quad\left(126 \mathrm{MHz}, \mathrm{CDCl}_{3}\right)$

159.05 (C(13)); 136.61 (C(3)); 130.21 (C(10)); 129.23 (2 x C(11)); 128.10 (C(2));

113.67 (2 x C(12)); 88.34 (C(5)); 74.86 (C(9)); 63.77 (C(1)); 55.24 (C(14)); 39.59

(C(4)); 30.95 (C(6)); 20.41 (C(7)); 17.51 (C(7')); 15.68 (C(9))

IR: (neat)

3392 (br, m); 2961 (s); 2871 (m); 1723 (s); 1689 (w); 1613 (m); 1587 (m); 1514

(s); 1464 (m); 1422 (m); 1383 (m); 1364 (m); 1351 (m); 1302 (m); 1249 (s); 1173

(m); $1110(\mathrm{~m}) ; 1037(\mathrm{~m}) ; 1012(\mathrm{~m})$

MS: $\quad\left(\mathrm{EI}, 70 \mathrm{eV}, 170^{\circ} \mathrm{C}\right)$

$278\left(0.3, \mathrm{M}^{+}\right) ; 206(2) ; 122(10) ; 121$ (100); 91 (3); 78 (5); 77 (4)

Opt. Rot.: $\quad[\alpha]_{\mathrm{D}}^{24}+4.9(\mathrm{c}=0.85, \mathrm{EtOH})$

TLC: $\quad R_{f} 0.18$ (hexanes/EtOAc 2/1) [silica gel, short UV]

Analysis: $\quad$ Calc for $\mathrm{C}_{17} \mathrm{H}_{26} \mathrm{O}_{3}(278.39)$

Calcd: $\quad$ C, $73.34 \% ; \quad H, 9.41 \%$

Found: $\quad$ C, $73.27 \% ; \quad H, 9.26 \%$

\section{(4S, 5S)-4,6-Dimethyl-5-(4-methoxyphenylmethoxy)-2-heptenal (3)}

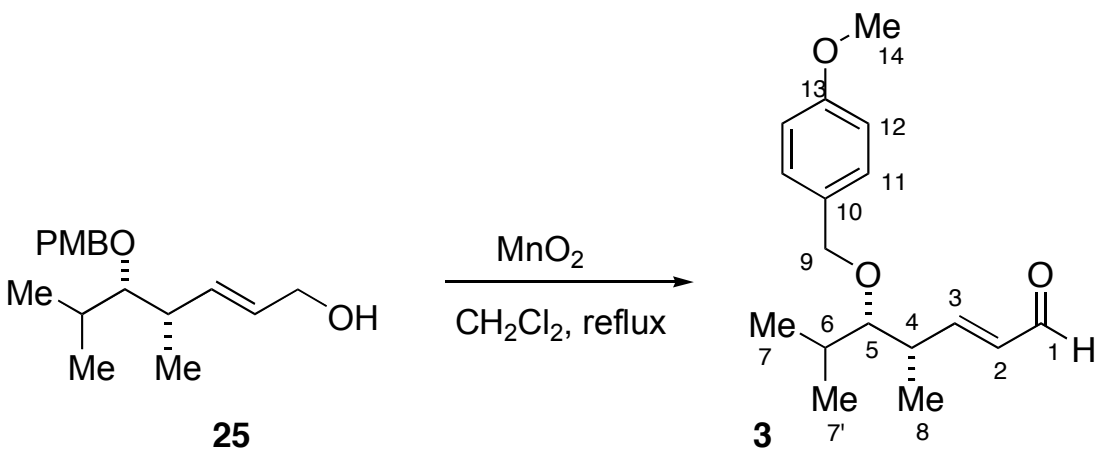

In a 250-mL round bottom flask were placed $25(8.07 \mathrm{~g}, 28.7 \mathrm{mmol})$, dichloromethane $(80 \mathrm{~mL})$ and manganese (IV) oxide (22.5 g, $259 \mathrm{mmol}, 9.0$ equiv). The flask was fitted with a reflux condenser and the reaction mixture was heated to reflux under argon for $36 \mathrm{~h}$. The 
reaction mixture was cooled to $\mathrm{rt}$ and was filtered through a pad of silica gel $(60 \mathrm{~mm} \times 2 \mathrm{~cm})$. The silica gel was washed with dichloromethane $(200 \mathrm{~mL})$ and ethyl acetate $(200 \mathrm{~mL})$. The combined filtrates were concentrated under reduced pressure. The resulting brown oil was chromatographed (silica gel, hexanes/EtOAc 5/1, $60 \mathrm{~mm}$ ) to afford $5.58 \mathrm{~g}(20.2 \mathrm{mmol}, 70 \%)$ of $\mathbf{3}$ as a pale yellow oil along with $2.30 \mathrm{~g}(8.27 \mathrm{mmol}, 29 \%)$ of recovered 25.

Data for 3:

${ }^{1} \mathrm{H} \mathrm{NMR}: \quad\left(500 \mathrm{MHz}, \mathrm{CDCl}_{3}\right)$

9.47 (d, $J=8.0,1 \mathrm{H}, \mathrm{HC}(1)) ; 7.25$ (d, $J=8.5,2 \mathrm{H}, 2$ x HC(11)); 6.88 (d, $J=8.6$, $2 \mathrm{H}, 2 \times \mathrm{HC}(12)) ; 6.83(\mathrm{dd}, J=15.8,7.8,1 \mathrm{H}, \mathrm{HC}(3)) ; 6.12(\mathrm{dd}, J=15.5,8.0,1$ $\mathrm{H}, \mathrm{HC}(2))$; $4.50\left(\mathrm{AB}, 2 \mathrm{H}, \mathrm{H}_{2} \mathrm{C}(9)\right) ; 3.81$ (s, $\left.3 \mathrm{H}, \mathrm{H}_{3} \mathrm{C}(14)\right) ; 3.13$ (t, $J=5.5,1 \mathrm{H}$, $\mathrm{HC}(5)$ ); 2.74 (sext, $J=6.2,1 \mathrm{H}, \mathrm{HC}(4)) ; 1.84$ (sext, $J=6.8,1 \mathrm{H}, \mathrm{HC}(6)) ; 1.15$ (d, $\left.J=6.5,3 \mathrm{H}, \mathrm{H}_{3} \mathrm{C}(9)\right) ; 0.97\left(\mathrm{~d}, J=6.5,3 \mathrm{H}, \mathrm{H}_{3} \mathrm{C}(7)\right) ; 0.97\left(\mathrm{~d}, J=6.6,3 \mathrm{H}, \mathrm{H}_{3} \mathrm{C}\left(7^{\prime}\right)\right.$

${ }^{13} \mathrm{C} \mathrm{NMR}: \quad\left(126 \mathrm{MHz}, \mathrm{CDCl}_{3}\right)$

194.45 (C(1)); 162.28 (C(3)); 159.49 (C(13)); 132.02 (C(2)); 130.82 (C(10)); 129.61 (2 x C(11)); 114.02 (2 x C(12)); 87.39 (C(5)); 74.93 (C(9)); 55.48 (C(14)); 40.26 (C(4)); 31.56 (C(6)); 20.44 (C(7)); 18.16 (C(7’)); 14.41 (C(9)

IR: (neat)

2962 (m); $2873(\mathrm{~m}) ; 2837(\mathrm{~m}) ; 2731(\mathrm{w}) ; 1686(\mathrm{~s}) ; 1612(\mathrm{~m}) ; 1586(\mathrm{~m}) ; 1514$ (m); 1466(m); $1421(\mathrm{w}) ; 1384$ (m); 1364 (m); 1350 (m); 1302 (m); 1248 (m); $1173(\mathrm{~m}) ; 1112(\mathrm{~m}) ; 1059(\mathrm{~m}) ; 1036$ (m)

$\underline{\mathrm{MS}}: \quad$ (FI)

277 (20); $276\left(100, \mathrm{M}^{+}\right)$

Opt. Rot.: $\quad[\alpha]_{D}^{24}+19.6(\mathrm{c}=0.45, \mathrm{EtOH})$

TLC: $\quad R_{f} 0.18$ (hexanes/EtOAc 5/1) [silica gel, $p$-anisaldehyde]

Analysis: $\quad$ Calc for $\mathrm{C}_{17} \mathrm{H}_{26} \mathrm{O}_{3}(276.37)$

Calcd: $\quad$ C, $73.88 \% ; \quad H, 8.75 \%$

Found: $\quad$ C, $73.52 \% ; \quad H, 8.72 \%$ 
(7S, 9S, 2' $\left.R, 4^{\prime} S, 6^{\prime} R\right)$ 1-(6'-(2-benzyldimethylsilyl-ethenyl)-2'-phenyl-[1,3]-dioxan-4'-yl)7,9-dimethyl-6-hydroxy-8-(4-methoxy-phenylmethoxy)-5-nonen-2-one (12)

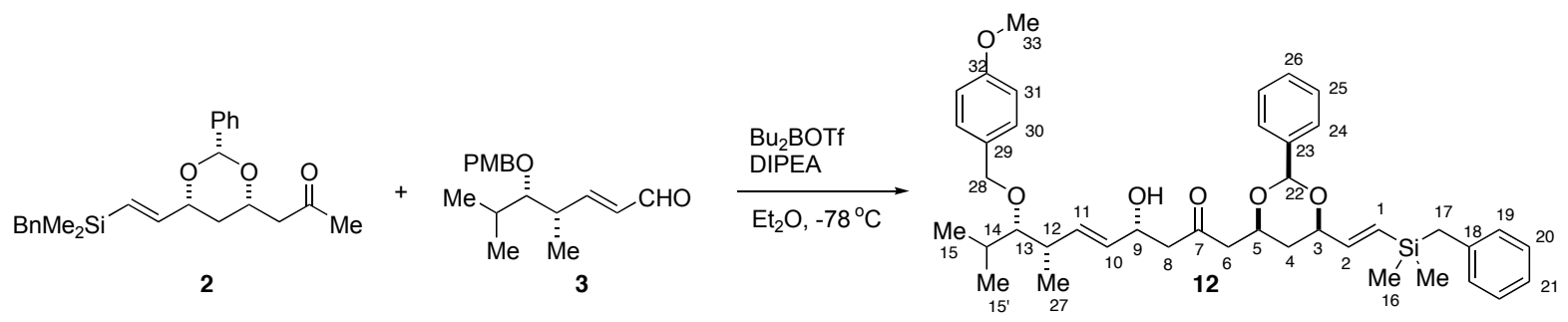

In a 50-mL Schlenk flask were placed 2 (592 mg, $1.50 \mathrm{mmol}, 1.2$ equiv) and ether (7 $\mathrm{mL}$ ). The solution was cooled to $-72{ }^{\circ} \mathrm{C}$ (internal temperature) using a dry ice/isopropanol bath. To the solution was added diisopropylethylamine $(0.32 \mathrm{~mL}, 1.80 \mathrm{mmol}, 1.44$ equiv $)$ followed by a 1.0 M solution of dibutylboron triflate in dichloromethane (1.65 mL, $1.65 \mathrm{mmol}, 1.32 \mathrm{equiv})$. The reaction mixture was stirred at $-72{ }^{\circ} \mathrm{C}$ for $15 \mathrm{~min}$ before the addition of 3 (345 $\mathrm{mg}, 1.25$ mmol) whereupon the reaction mixture was stirred for $7 \mathrm{~h}$. To the reaction mixture was added a 6/1 $\mathrm{MeOH} / \mathrm{pH} 7$ phosphate buffer solution $(10 \mathrm{~mL})$. The reaction mixture was warmed to $0{ }^{\circ} \mathrm{C}$ using an ice bath. To the reaction mixture was added a 3/1 $\mathrm{MeOH} / 30 \% \mathrm{H}_{2} \mathrm{O}_{2}$ solution (10 mL) dropwise. The resulting emulsion was stirred for $1 \mathrm{~h}$ and was poured into a $125-\mathrm{mL}$ separatory funnel. The whole mixture was extracted with ethyl acetate $(3 \times 50 \mathrm{~mL})$. The combined extracts were washed with saturated aqueous $\mathrm{NaHCO}_{3}$ solution $(25 \mathrm{~mL})$ and brine $(25 \mathrm{~mL})$. The extracts were dried over $\mathrm{Na}_{2} \mathrm{SO}_{4}$ and were concentrated under reduced pressure. The resulting clear oil was chromatographed (silica gel, hexanes/EtOAc 5/1 to $3 / 1,40 \mathrm{~mm}$ ) to afford $715 \mathrm{mg}(1.07$ mmol, $85 \%$ ) of $\mathbf{1 2}$ as a clear, colorless oil.

Data for 12:

${ }^{1}$ H NMR: $\quad\left(500 \mathrm{MHz}, \mathrm{CDCl}_{3}\right)$

$7.49(\mathrm{dd}, J=7.7,1.8,2 \mathrm{H}, 2$ x $\mathrm{HC}(24)) ; 7.32-7.38(\mathrm{~m}, 3 \mathrm{H}, \mathrm{HC}(26), 2$ х $\mathrm{HC}(25)) ; 7.27$ (d, $J=7.5,2 \mathrm{H}, 2$ x $\mathrm{HC}(30)) ; 7.20$ (t, $J=7.6,2 \mathrm{H}, 2$ x $\mathrm{HC}(20))$; 7.07 (t, $J=7.3,1 \mathrm{H}, \mathrm{HC}(21)) ; 6.99$ (dd, $J=7.3,1.3,2 \mathrm{H}, 2 \times \mathrm{HC}(19)) ; 6.86$ (d, $J$ $=8.5,2 \mathrm{H}, 2 \times \mathrm{HC}(31)) ; 6.06(\mathrm{dd}, J=18.9,4.5,1 \mathrm{H}, \mathrm{HC}(2)) ; 5.92(\mathrm{dd}, J=19.1$, 1.3, $1 \mathrm{H}, \mathrm{HC}(1)) ; 5.67$ (ddd, $J=15.5,8.0,1.0,1 \mathrm{H}, \mathrm{HC}(11)$ ); 5.59 (s, $1 \mathrm{H}$, 
$\mathrm{HC}(22)) ; 5.46$ (ddd, $J=15.5,6.3,0.8,1 \mathrm{H}, \mathrm{HC}(10)) ; 4.52-4.57$ (m, $1 \mathrm{H}, \mathrm{HC}(9))$; 4.48 (s, $\left.2 \mathrm{H}, \mathrm{H}_{2} \mathrm{C}(28)\right) ; 4.35$ - 4.41 (m, $\left.2 \mathrm{H}, \mathrm{HC}(3), \mathrm{HC}(5)\right) ; 3.79$ (s, $3 \mathrm{H}$, $\left.\mathrm{H}_{3} \mathrm{C}(33)\right) ; 2.95(\mathrm{t}, J=5.8, \mathrm{HC}(13)) ; 2.75(\mathrm{~d}, J=3.7, \mathrm{HO}) ; 2.73\left(\mathrm{ABX}, J_{\mathrm{AB}}=16.3\right.$, $\left.J_{\mathrm{AX}}=7.3, J_{\mathrm{BX}}=5.4,2 \mathrm{H}, \mathrm{H}_{2} \mathrm{C}(6)\right) ; 2.67\left(\mathrm{ABX}, J_{\mathrm{AB}}=11.5, J_{\mathrm{AX}}=5.9, J_{\mathrm{BX}}=3.1,2\right.$ $\mathrm{H}, \mathrm{H}_{2} \mathrm{C}(8)$ ); 2.41 (sext, $J=6.9,1 \mathrm{H}, \mathrm{HC}(12)$ ); 2.13 (s, $2 \mathrm{H}, \mathrm{H}_{3} \mathrm{C}(17)$ ); 1.79 (sext, $J$ $=6.8,1 \mathrm{H}, \mathrm{HC}(14)) ; 1.75\left(\mathrm{dt}, J=12.9,2.4,1 \mathrm{H}, \mathrm{H}_{\mathrm{eq}} \mathrm{C}(4)\right) ; 1.47(\mathrm{dt}, J=13.2,11.2$, $\left.1 \mathrm{H}, \mathrm{H}_{\mathrm{ax}} \mathrm{C}(4)\right) ; 1.05\left(\mathrm{~d}, J=6.8,3 \mathrm{H}, \mathrm{H}_{3} \mathrm{C}(27)\right) ; 0.94$ (d, $\left.J=8.3,3 \mathrm{H}, \mathrm{H}_{3} \mathrm{C}(15)\right)$; $0.92\left(\mathrm{~d}, J=8.3,3 \mathrm{H}, \mathrm{H}_{3} \mathrm{C}\left(15^{\prime}\right)\right)$; 0.04, 0.04 (s, $6 \mathrm{H}, 2$ x $\left.\mathrm{H}_{3} \mathrm{C}(14)\right)$

${ }^{13} \mathrm{C} \mathrm{NMR:} \quad\left(126 \mathrm{MHz}, \mathrm{CDCl}_{3}\right)$

$208.73(\mathrm{C}(7))$; $159.00(\mathrm{C}(32)) ; 145.56(\mathrm{C}(2)) ; 139.71(\mathrm{C}(18)) ; 138.13(\mathrm{C}(23))$; 135.63 (C(11)); 131.17 (C(29)); 129.73 (C(10)); 129.17 (C(30)); 128.55 (C(26)); 128.31 (C(1)); 128.22 (2 x C(19), 2 x C(25)); 128.09 (2 x C(20)); 126.12 (2 x $\mathrm{C}(24)) ; 123.99(\mathrm{C}(21)) ; 113.65(\mathrm{C}(31)) ; 100.59$ (C(22)); 88.25 (C(13)); 78.37 $(\mathrm{C}(3))$; $74.86(\mathrm{C}(9)) ; 72.72(\mathrm{C}(5))$; 68.33 (C(28)); 55.24 (C(33)); 50.50 (C(8)); 49.30 (C(6)); 39.61 (C(12)); 36.28 (C(4)); 30.97 (C(14)); 25.81 (C(17)); 20.41, 17.44 (C(15), C(15')); 15.70 (C(27)); -3.57, -3.60 (2 x C(16))

IR: (neat)

3480 (br, m); 3061 (m); 3025 (m); 2959(s); 2872 (m); 1714 (s); 1614 (m); 1601 (m); 1589 (m); 1514 (s); 1494 (m); 1454(m); 1384 (m); 1338 (m); 1302 (s); 1248 (m); $1209(\mathrm{~m}) ; 1174(\mathrm{~m}) ; 1030(\mathrm{~m})$

MS: $\quad$ (HR ESI)

Calcd: 693.3587 (for $\mathrm{C}_{41} \mathrm{H}_{54} \mathrm{O}_{6} \mathrm{Si}+\mathrm{Na}$ )

Found: 693.3603

Opt. Rot.: $\quad[\alpha]_{\mathrm{D}}^{24}+8.5(\mathrm{c}=0.45$, EtOH $)$

TLC: $\quad R_{f} 0.09$ (hexane/EtOAc 3/1) [silica gel, $p$-anisaldehyde]

Analysis: $\quad$ Calc for $\mathrm{C}_{41} \mathrm{H}_{54} \mathrm{O}_{6} \mathrm{Si}$ (670.95)
Calcd:
C, $73.39 \%$;
$\mathrm{H}, 8.11 \%$

Found:

C, $73.33 \%$;

H, $7.97 \%$ 
Determination of Configuration at C(9) in Aldol Adduct 12, (C(27) in RK-397).

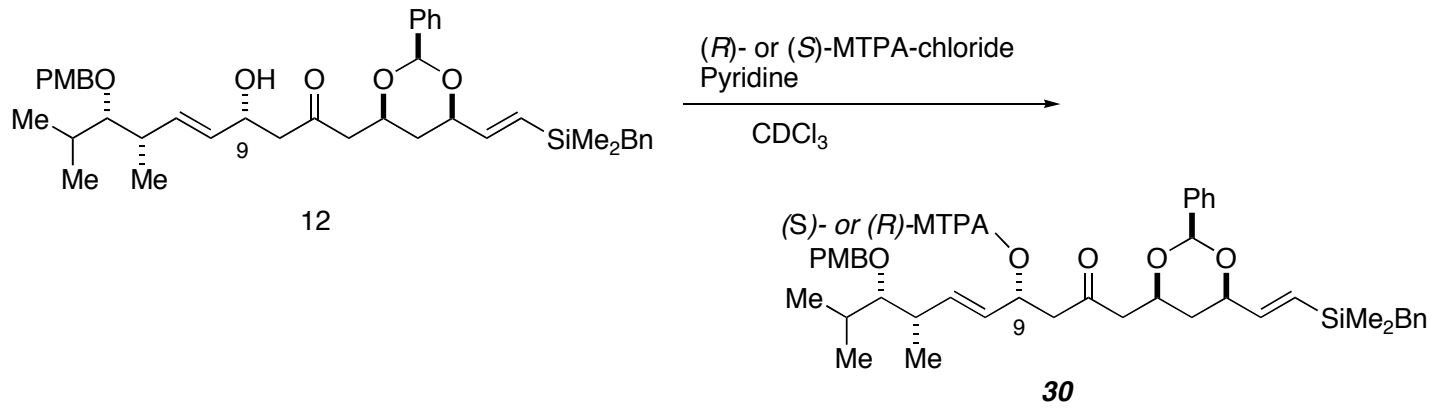

In an NMR tube was placed a solution of $12(5.0 \mathrm{mg}, 0.0075 \mathrm{mmol})$ in $\mathrm{CDCl}_{3}(0.75 \mathrm{~mL})$. To the solution was added sequentially pyridine $(0.02 \mathrm{~mL})$ and $(R)$ - or $(S)$ - $\alpha$-methoxy- $\alpha$ trifluoromethylphenylacetyl chloride $(2.8 \mu \mathrm{L}, 0.015 \mathrm{mmol}, 2.0$ equiv). The reaction mixture was thoroughly mixed and was let stand for $2 \mathrm{~h}$ at $\mathrm{rt}$. The crude Mosher ester $\mathbf{3 0}$ was analyzed by NMR and found to be of $R$ configuration.

From $(R)$-MTPA-30:

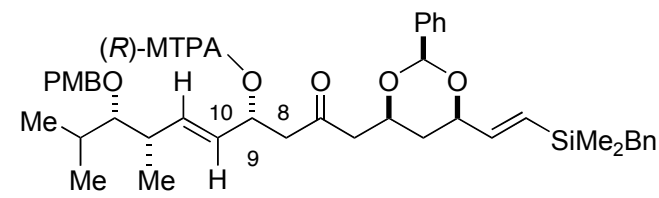

$\delta \mathrm{H}_{2} \mathrm{C}(8)=2.70 \mathrm{ppm}$

$\delta \mathrm{HC}(10)=5.48 \mathrm{ppm}$
From $(S)$-MTPA-30:

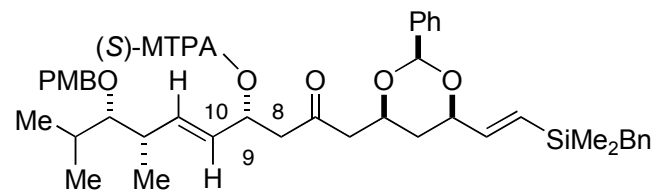

$\delta \mathrm{H}_{2} \mathrm{C}(8)=2.77 \mathrm{ppm}$

$\delta \mathrm{HC}(10)=5.35 \mathrm{ppm}$

Difference in chemical shifts:

$\Delta \delta_{S R} \mathrm{H}_{2} \mathrm{C}(8)=+0.07 \mathrm{ppm}$

$\Delta \delta_{S R} \mathrm{HC}(9)=-0.13 \mathrm{ppm}$ 


\section{Bisbenzylidene Silane 26}
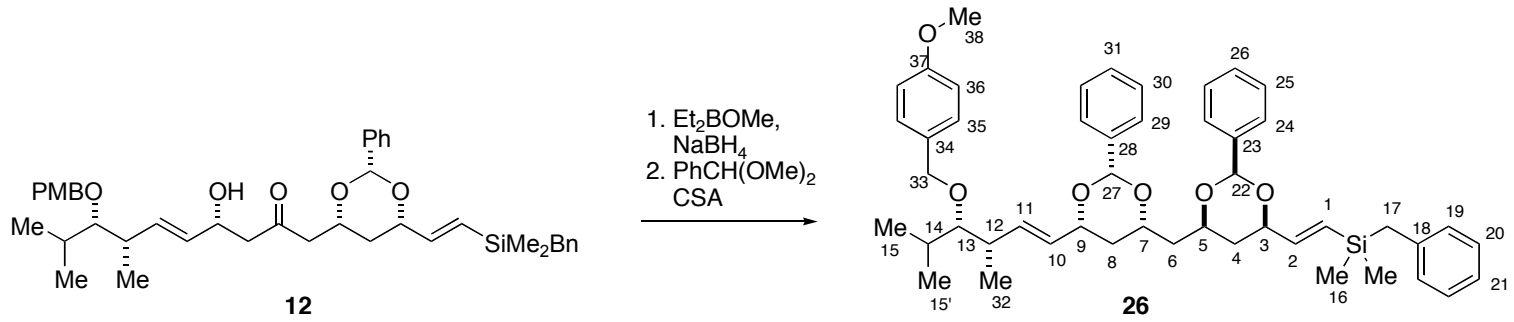

In a 50-mL Schlenk flask were placed $12(2.85 \mathrm{~g}, 4.25 \mathrm{mmol})$, tetrahydrofuran $(20 \mathrm{~mL})$ and methanol $(4.3 \mathrm{~mL})$. The reaction mixture was cooled to $-72{ }^{\circ} \mathrm{C}$ (internal temperature) using dry ice/isopropanol bath. To the solution was added dropwise diethylmethoxyborane $(0.693 \mathrm{~mL}$, $5.10 \mathrm{mmol}, 1.2$ equiv), and the reaction mixture was stirred for $15 \mathrm{~min}$ at $-72{ }^{\circ} \mathrm{C}$. To the solution was added sodium borohydride (193 mg, $5.10 \mathrm{mmol}, 1.2$ equiv) and the mixture was stirred for $4 \mathrm{~h}$ at $-72{ }^{\circ} \mathrm{C}$. The reaction mixture was quenched with acetic acid $(6 \mathrm{~mL})$ in one portion and the mixture was allowed to warm to $\mathrm{rt}$ and then was transferred to a $125-\mathrm{mL}$ separatory funnel. The solution was extracted with ethyl acetate $(2 \times 25 \mathrm{~mL})$ and the combined organic layers were washed with saturated aqueous $\mathrm{NaHCO}_{3}$ solution $(20 \mathrm{~mL})$ and brine $(20 \mathrm{~mL})$. The extracts were dried over $\mathrm{Na}_{2} \mathrm{SO}_{4}$ and were concentrated under reduced pressure to afford a crude diol (3.10 g). In a $100-\mathrm{mL}$ round bottom flask were placed the crude diol $(3.10 \mathrm{~g})$, dichloromethane $(25 \mathrm{~mL})$, benzaldehyde dimethyl acetal $(2.54 \mathrm{~mL}, 17.0 \mathrm{mmol}, 4.0$ equiv) and $d l$-camphorsulfonic acid (148 $\mathrm{mg}, 0.64 \mathrm{mmol}, 0.15$ equiv). The reaction mixture was stirred at $\mathrm{rt}$ for $16 \mathrm{~h}$ and was quenched with saturated aqueous $\mathrm{NaHCO}_{3}$ solution $(30 \mathrm{~mL})$. The biphasic mixture was transferred to a $125-\mathrm{mL}$ separatory funnel and the aqueous layer was extracted with dichloromethane $(20 \mathrm{~mL})$. The combined organic layers were washed with saturated aqueous $\mathrm{NaHCO}_{3}$ solution $(20 \mathrm{~mL})$ and brine $(20 \mathrm{~mL})$. The solution was dried over $\mathrm{Na}_{2} \mathrm{SO}_{4}$ and concentrated under reduced pressure. The resulting crude oil was chromatographed (silica gel, hexanes/ether 9/1, $60 \mathrm{~mm})$ to afford $2.79 \mathrm{~g}(3.80 \mathrm{mmol}, 86 \%)$ of $\mathbf{2 6}$ as a clear, colorless oil.

\section{Data for 26:}

${ }^{1} \mathrm{H}$ NMR: $\quad\left(500 \mathrm{MHz}, \mathrm{CDCl}_{3}\right)$

$7.55(\mathrm{dd}, J=8.3,1.5,2 \mathrm{H}, 2 \times \mathrm{HC}(24)$ or $\mathrm{HC}(29)) ; 7.53(\mathrm{dd}, J=8.1,1.3,2 \mathrm{H}, 2 \mathrm{x}$ 
$\mathrm{HC}(24)$ or $\mathrm{HC}(29)) ; 7.34-7.40$ (m, $6 \mathrm{H}, \mathrm{HC}(26), 2$ x $\mathrm{HC}(25), \mathrm{HC}(31), 2$ x $\mathrm{HC}(30)) ; 7.27$ (d, $J=7.3,2 \mathrm{H}, 2$ x HC(35)); 7.19 (t, $J=7.6,2 \mathrm{H}, 2$ x HC(20)); $7.06(\mathrm{t}, J=7.4,1 \mathrm{H}, \mathrm{HC}(21)) ; 6.98$ (d, $J=6.8,2 \mathrm{H}, 2$ x HC(19)); 6.83 (d, $J=8.6$, $2 \mathrm{H}, 2 \times \mathrm{HC}(36)) ; 6.07(\mathrm{dd}, J=19.0,4.8,1 \mathrm{H}, \mathrm{HC}(2)) ; 5.91(\mathrm{dd}, J=19.0,1.4,1$ $\mathrm{H}, \mathrm{HC}(1)) ; 5.76(\mathrm{dd}, 15.7,7.9,1 \mathrm{H}, \mathrm{HC}(11)) ; 5.61$ (s, $1 \mathrm{H}, \mathrm{HC}(22)$ or $\mathrm{HC}(27))$; 5.61 (s, $1 \mathrm{H}, \mathrm{HC}(22)$ or $\mathrm{HC}(27)) ; 5.59$ (dd, $J=16.7,6.9,1 \mathrm{H}, \mathrm{HC}(10)) ; 4.48$ (AB, $\left.J_{\mathrm{ab}}=10.8,2 \mathrm{H}, \mathrm{H}_{2} \mathrm{C}(33)\right) ; 4.31-4.36(\mathrm{~m}, 2 \mathrm{H}, \mathrm{HC}(3), \mathrm{HC}(9)) ; 4.20-4.23$ (m, 2 $\mathrm{H}, \mathrm{HC}(5), \mathrm{HC}(7)) ; 3.78$ (s, $3 \mathrm{H}, \mathrm{H}_{3} \mathrm{C}(38)$ ); 2.98 (t, $J=5.8, \mathrm{HC}(13)$ ); 2.45 (sext, $J$ = 6.7, $1 \mathrm{H}, \mathrm{HC}(12)$ ); 2.13 (s, $2 \mathrm{H}, \mathrm{H}_{3} \mathrm{C}(17)$ ); 1.83 (sext, $J=6.7,1 \mathrm{H}, \mathrm{HC}(14)$ ); $1.81\left(\mathrm{~m}, 2 \mathrm{H}, \mathrm{H}_{2} \mathrm{C}(6)\right) 1.66\left(\mathrm{dt}, J=13.2,2.5,1 \mathrm{H}, \mathrm{H}_{\mathrm{eq}} \mathrm{C}(4)\right.$ or $\left.\mathrm{H}_{\mathrm{eq}} \mathrm{C}(8)\right)$ ) 1.63 (dt, $J$ $=13.2,2.6,1 \mathrm{H}, \mathrm{H}_{\mathrm{eq}} \mathrm{C}(4)$ or $\left.\mathrm{H}_{\mathrm{eq}} \mathrm{C}(8)\right) ; 1.54\left(\mathrm{dt}, J=13.5,11.2,1 \mathrm{H}, \mathrm{H}_{\mathrm{ax}} \mathrm{C}(4)\right.$ or $\mathrm{H}_{\mathrm{ax}} \mathrm{C}(8)$ ); 1.49 (dt, $J=13.5,11.2,1 \mathrm{H}, \mathrm{H}_{\mathrm{ax}} \mathrm{C}(4)$ or $\left.\mathrm{H}_{\mathrm{ax}} \mathrm{C}(8)\right) ; 1.07$ (d, $J=6.8,3 \mathrm{H}$, $\left.\mathrm{H}_{3} \mathrm{C}(32)\right)$; 0.95 (d, $\left.J=6.6,3 \mathrm{H}, \mathrm{H}_{3} \mathrm{C}(15)\right)$; 0.94 (d, $J=6.9,3 \mathrm{H}, \mathrm{H}_{3} \mathrm{C}\left(15^{\prime}\right)$ ); 0.04 (s, $6 \mathrm{H}, 2 \times \mathrm{H}_{3} \mathrm{C}(14)$ )

${ }^{13} \mathrm{C} \mathrm{NMR}: \quad\left(126 \mathrm{MHz}, \mathrm{CDCl}_{3}\right)$ 158.94 (C(37)); 146.08 (C(2)); 139.67 (C(18)); 138.76, 138.66 (C(23), C(27)); 135.93 (C(11)); 131.07 (C(34)); 129.20 (2 x C(35)); 128.81 (C(10)); 128.62, 128.55 (C(26), C(31)); 128.15 (2 x C(25) or C(30)); 128.11 (2 x C(19)); 128.10 (2 x C(25) or $\mathrm{C}(30)) ; 128.01$ ( 2 x C(20)); 127.97 (C(1)); 126.15 (2 x C(24), 2 x $\mathrm{C}(29)) ; 123.92$ (C(21)); 113.57 (2 x C(31)); 100.38, 100.33 (C(22), C(27)); 88.23 (C(13)); 78.47 (C(3)); 77.15 (C(9)); 74.78 (C(33)); 72.36, 72.33 (C(5), C(7)); 55.09 (C(38)); 42.42 (C(6)); 39.44 (C(12)); 37.49, $36.94(\mathrm{C}(4), \mathrm{C}(8))$; 30.91 (C(14)); 25.77 (C(17)); 20.20, 17.78 (C(15), C(15’)); 15.04 (C(32)); -3.61, -3.66 $(2 \times \mathrm{C}(16))$

IR: $\quad\left(\mathrm{CHCl}_{3}\right)$

3154 (w); 3066 (m); 3027 (m); 2959(s); 2916 (m); 2873 (m); 2838 (m); 2250 (m); 1794 (w); 1613 (m); 1600 (m); 1587 (w); 1514 (s); 1493 (m); 1453 (m); 1429 (w); 1408 (m); 1386 (m); 1339 (m); 1302 (m); 1248(s); 1210 (m); 1174 (m); 1121 (m); 1095 (m); 1057 (m); 1029 (s); 1010 (s) 
MS: $\quad(H R$ ESI)

Calcd: 783.4057 (for $\mathrm{C}_{48} \mathrm{H}_{60} \mathrm{O}_{6} \mathrm{Si}+\mathrm{Na}$ )

Found: 783.4059

Opt. Rot.: $\quad[\alpha]_{\mathrm{D}}^{24}+62.4(\mathrm{c}=1.05, \mathrm{EtOH})$

TLC: $\quad R_{f} 0.07$ (hexane/EtOAc 9/1) [silica gel, $p$-anisaldehyde]

Analysis: $\quad$ Calc for $\mathrm{C}_{48} \mathrm{H}_{60} \mathrm{O}_{6} \mathrm{Si}$

$\begin{array}{lll}\text { Calcd: } & \text { C }, 75.75 \% ; & \text { H, } 7.95 \% \\ \text { Found: } & \text { C, } 75.79 \% ; & \text { H, } 8.02 \%\end{array}$

\section{Bisbenzylidene Aldehyde 14}

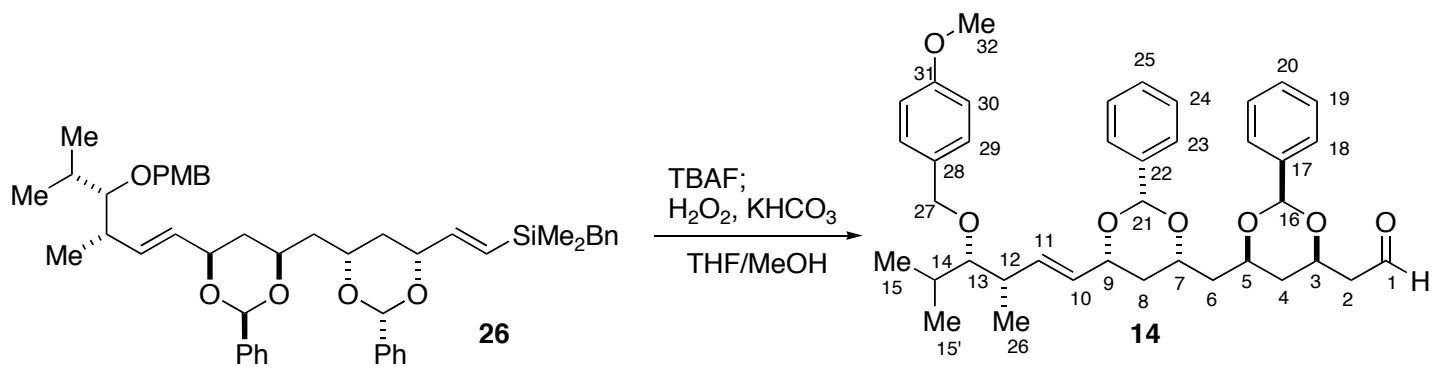

In a 100-mL round bottom flask were placed $26(751 \mathrm{mg}, 0.99 \mathrm{mmol})$ and tetrahydrofuran $(14 \mathrm{~mL})$. The solution was cooled to $0{ }^{\circ} \mathrm{C}$ (bath temperature) using an ice bath. To the solution was added a 1.0 M solution of TBAF (1.08 mL, $1.08 \mathrm{mmol}, 1.1$ equiv) dropwise over $10 \mathrm{~min}$. The reaction mixture was stirred for $15 \mathrm{~min}$ at $0{ }^{\circ} \mathrm{C}$. To the reaction mixture were added $30 \% \mathrm{H}_{2} \mathrm{O}_{2}$ solution (4.24 mL, $23.8 \mathrm{mmol}, 24$ equiv), methanol (10 mL) and $\mathrm{KHCO}_{3}(495$ $\mathrm{mg}, 4.95 \mathrm{mmol}, 5.0$ equiv). The reaction mixture was warmed to $\mathrm{rt}$ and stirred for $18 \mathrm{~h}$. To the reaction mixture was added water $(40 \mathrm{~mL})$ and the resulting emulsion was stirred for $30 \mathrm{~min}$. The reaction mixture was diluted with ethyl acetate $(20 \mathrm{~mL})$ and transferred to a $250-\mathrm{mL}$ separatory funnel. The aqueous layer was extracted with ethyl acetate $(2 \times 50 \mathrm{~mL})$ and the combined organic extracts were washed with $30 \%$ aqueous $\mathrm{Na}_{2} \mathrm{~S}_{2} \mathrm{O}_{3}$ solution $(3 \times 30 \mathrm{~mL})$ and with brine $(30 \mathrm{~mL})$. The combined extracts were dried over $\mathrm{MgSO}_{4}$ and concentrated. The resulting clear oil was chromatographed (silica gel, hexanes/ether 1/1, $30 \mathrm{~mm}$ ) to afford 14 (457 $\mathrm{mg}, 0.73 \mathrm{mmol}, 73 \%$ ) as white foamy solid. 


\section{Data for 14:}

mp: $\quad 101-103{ }^{\circ} \mathrm{C}$

${ }^{1} \mathrm{H} \mathrm{NMR}: \quad\left(500 \mathrm{MHz}, \mathrm{CDCl}_{3}\right)$

9.85 (t, $J=1.9,1 \mathrm{H}, \mathrm{HC}(1)) ; 7.52$ (dd, $J=8.0,1.6,2 \mathrm{H}, 2$ x HC(18) or HC(23)); 7.49 (dd, $J=7.9,1.5,2 \mathrm{H}, 2$ x HC(18) or HC(23)); $7.39-7.27$ (m, $6 \mathrm{H}, \mathrm{HC}(20)$, $2 \times \mathrm{HC}(19), \mathrm{HC}(25), 2 \times \mathrm{HC}(24)) ; 7.26$ (d, $J=8.3,2 \mathrm{H}, 2 \times \mathrm{HC}(29)) ; 6.84$ (d, $J=$ 8.8, 2 H, 2 x HC(30)) ; $5.76(\mathrm{dd}, J=15.6,8.0,1 \mathrm{H}, \mathrm{HC}(11)) ; 5.61$ (s, $1 \mathrm{H}, \mathrm{HC}(16)$ or $\mathrm{HC}(21)) ; 5.60(\mathrm{~s}, 1 \mathrm{H}, \mathrm{HC}(16)$ or $\mathrm{HC}(21)) ; 5.59(\mathrm{dd}, J=16.4,6.9,1 \mathrm{H}$, $\mathrm{HC}(10)) ; 4.48\left(\mathrm{AB}, J_{\mathrm{ab}}=10.6,2 \mathrm{H}, \mathrm{H}_{2} \mathrm{C}(27)\right)$; 4.45 - 4.41 (m, $\left.1 \mathrm{H}, \mathrm{HC}(9)\right)$; 4.34 $4.31(\mathrm{~m}, 1 \mathrm{H}, \mathrm{HC}(3)$ or $\mathrm{HC}(5)$ or $\mathrm{HC}(7)) ; 4.26-4.18(\mathrm{~m}, 2 \mathrm{H}, \mathrm{HC}(3)$ or $\mathrm{HC}(5)$ or $\mathrm{HC}(7)) ; 3.78\left(\mathrm{~s}, 3 \mathrm{H}, \mathrm{H}_{3} \mathrm{C}(32)\right) ; 2.98(\mathrm{t}, J=5.8, \mathrm{HC}(13)) ; 2.71\left(\mathrm{ABXd}, J_{\mathrm{ax}}=7.4\right.$, $\left.J_{\mathrm{bx}}=5.1, J_{\mathrm{ab}}=16.9, J=2.0,2 \mathrm{H}, \mathrm{H}_{2} \mathrm{C}(2)\right) ; 2.44$ (sext, $J=6.8,1 \mathrm{H}, \mathrm{HC}(12)$ ); 1.83 (sext, $J=6.6,1 \mathrm{H}, \mathrm{HC}(14)) ; 1.82-1.80\left(\mathrm{~m}, 2 \mathrm{H}, \mathrm{H}_{2} \mathrm{C}(6)\right) 1.71(\mathrm{dt}, J=13.0,2.5,1$ $\mathrm{H}, \mathrm{H}_{\mathrm{eq}} \mathrm{C}(4)$ or $\left.\mathrm{H}_{\mathrm{eq}} \mathrm{C}(8)\right)$; $1.62\left(\mathrm{dt}, J=13.2,2.6,1 \mathrm{H}, \mathrm{H}_{\mathrm{eq}} \mathrm{C}(4)\right.$ or $\left.\mathrm{H}_{\mathrm{eq}} \mathrm{C}(8)\right) ; 1.57$ $1.43\left(\mathrm{~m}, 2 \mathrm{H}, \mathrm{H}_{\mathrm{ax}} \mathrm{C}(4), \mathrm{H}_{\mathrm{ax}} \mathrm{C}(8)\right) ; 1.06\left(\mathrm{~d}, J=6.9,3 \mathrm{H}, \mathrm{H}_{3} \mathrm{C}(26)\right) ; 0.95$ (d, $J=6.6$, $\left.3 \mathrm{H}, \mathrm{H}_{3} \mathrm{C}(15)\right) ; 0.94$ (d, $J=6.8,3 \mathrm{H}, \mathrm{H}_{3} \mathrm{C}\left(15^{\prime}\right)$ )

${ }^{13} \mathrm{C} \mathrm{NMR}: \quad\left(126 \mathrm{MHz}, \mathrm{CDCl}_{3}\right)$

200.37 (C(1)); 159.03 (C(31)); 138.74, 138.31 (C(22), C(17)); 136.11 (C(11)); 131.18 (C(28)); 129.30 (2 x C(29)); 128.82 (C(10)); 128.67 (C(20), C(25)); 128.24, 128.20 ( 2 x C(19), 2 x C(24)); 126.19, 126.02 (2 x C(18), 2 x C(23)); 113.66 (2 x C(30)); 100.59, 100.46 (C(16), C(21)); 88.35 (C(13)); 77.25 (C(9)); 74.87 (C(27)); 72.45, 72.33, 71.81 (C(3), C(5), C(7)); 55.23 (C(32)); 49.30 (C(2)); 42.37 (C(6)); 39.54 (C(12)); 37.53, 37.00 (C(4), C(8)); 30.99 (C(14)); 20.26, $17.81\left(\mathrm{C}(15), \mathrm{C}\left(15^{\prime}\right)\right) ; 15.12(\mathrm{C}(32))$

IR: $\quad\left(\mathrm{CH}_{2} \mathrm{Cl}_{2}\right)$

3051 (m); 2960(s); 2911 (m); 2869 (m); 1726 (w); 1612 (m); 1600 (m); 1514 (m); 1458 (m); 1344 (m); 1266 (m); 1244(m); 1110 (m); 1058 (m); 1028 (m); 1007 (m)

MS: $\quad(F D)$

630 (15); 629 (47); 628 (100, M+); 627 (12); 556 (17); 435 (14); 122 (10); 121 (69); 105 (22)

Opt. Rot.: $\quad[\alpha]_{\mathrm{D}}^{24}+81.3(\mathrm{c}=0.45, \mathrm{EtOH})$ 
TLC: $\quad R_{f} 0.12$ (hexane/Ether 1/1) [silica gel, $p$-anisaldehyde]

Analysis: $\quad$ Calc for $\mathrm{C}_{39} \mathrm{H}_{48} \mathrm{O}_{7}$ (628.79)
Calcd:
C, $74.49 \%$;
$\mathrm{H}, 7.69 \%$
Found:
C, $74.55 \%$;
$\mathrm{H}, 7.67 \%$

\section{Aldol Product 15}
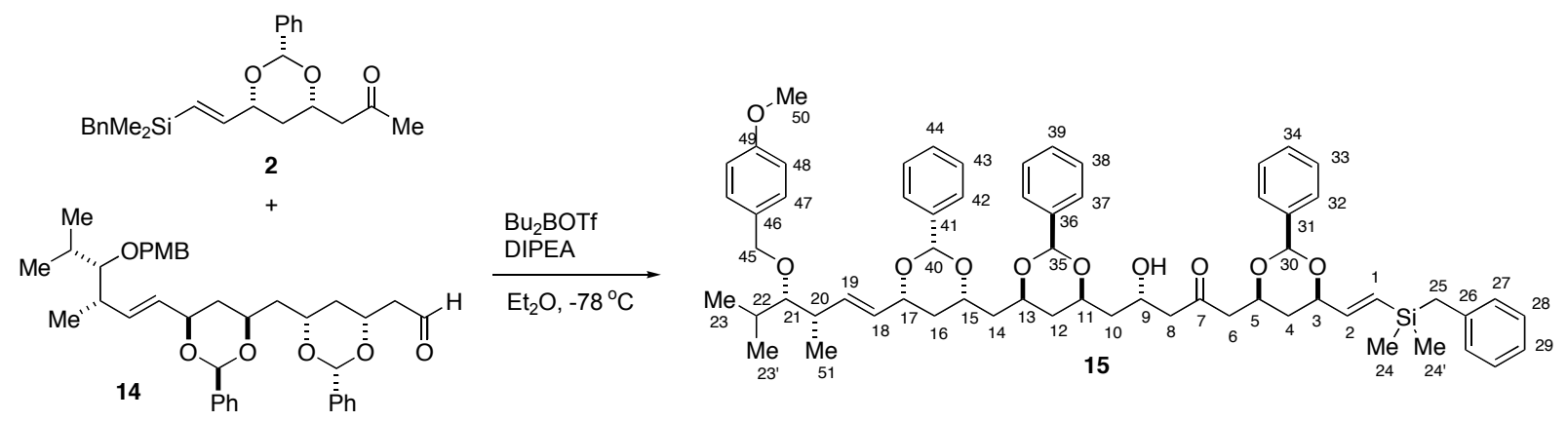

In a 100-mL Schlenk flask were placed $2(1.07 \mathrm{~g}, 2.71 \mathrm{mmol}, 1.2$ equiv) and ether (10 $\mathrm{mL}$ ). The solution was cooled to $-72{ }^{\circ} \mathrm{C}$ (internal temperature) using a dry ice/isopropanol bath. To the reaction mixture were added diisopropylethylamine $(0.583 \mathrm{~mL}, 3.25 \mathrm{mmol}, 1.44$ equiv) followed by a $1.0 \mathrm{M}$ solution of dibutylboron triflate $(2.98 \mathrm{~mL}, 2.98 \mathrm{mmol}, 1.32$ equiv) dropwise. The reaction mixture was stirred for $20 \mathrm{~min}$ before the addition of solution of 14 (1.42 $\mathrm{g}, 2.26 \mathrm{mmol})$ in dichloromethane $(7 \mathrm{~mL}+2 \mathrm{~mL}$ rinse) dropwise over $1 \mathrm{~h}$ whereupon the reaction mixture was stirred for $8 \mathrm{~h}$. To the reaction mixture was added a $6 / 1$ mixture $\mathrm{MeOH} / \mathrm{pH}$ 7 phosphate buffer solution $(35 \mathrm{~mL})$. The resulting emulsion was allowed to warm to $0{ }^{\circ} \mathrm{C}$ using an ice bath. To the reaction mixture was added dropwise a 3/1 mixture of $\mathrm{MeOH} / 30 \% \mathrm{H}_{2} \mathrm{O}_{2}$ solution $(32 \mathrm{~mL})$. The reaction mixture was stirred for $30 \mathrm{~min}$ and was poured into a $250-\mathrm{mL}$ separatory funnel. The solution was diluted with ethyl acetate $(100 \mathrm{~mL})$ and was washed with water $(20 \mathrm{~mL})$, saturated aqueous $\mathrm{NaHCO}_{3}$ solution $(20 \mathrm{~mL})$ and brine $(20 \mathrm{~mL})$. The solution was dried over $\mathrm{Na}_{2} \mathrm{SO}_{4}$ and was concentrated under reduced pressure. The resulting clear oil was chromatographed (silica gel, hexanes/EtOAc 5/1 to 2/1, $50 \mathrm{~mm}$ ) to afford $2.03 \mathrm{~g}(1.98 \mathrm{mmol}$, $88 \%)$ of $\mathbf{1 5}$ as white foam. 


\section{Data for 15:}

mp: $\quad 52-53{ }^{\circ} \mathrm{C}$

${ }^{1} \mathrm{H} \mathrm{NMR}: \quad\left(500 \mathrm{MHz}, \mathrm{CDCl}_{3}\right)$

$7.53-7.46$ (m, $6 \mathrm{H}, 2$ x HC(32), 2 x HC(37), 2 x HC(42)); $7.38-7.30$ (m, $9 \mathrm{H}$, HC(34), 2 x HC(33), HC(39), 2 x HC(38), HC(44), 2 x HC(43)); 7.26 (d, J = 7.3, $2 \mathrm{H}, 2 \times \mathrm{HC}(47)) ; 7.20(\mathrm{t}, J=7.7,2 \mathrm{H}, 2 \times \mathrm{HC}(28)) ; 7.07(\mathrm{t}, J=7.3,1 \mathrm{H}$, $\mathrm{HC}(29)) ; 6.98$ (d, $J=7.6,2 \mathrm{H}, 2$ x HC(27)); 6.84 (d, $J=8.6,2 \mathrm{H}, 2$ x HC(48) ; 6.05 (dd, $J=18.9,4.5,1 \mathrm{H}, \mathrm{HC}(2)) ; 5.93$ (d, $J=18.8,1 \mathrm{H}, \mathrm{HC}(1)) ; 5.77$ (dd, 15.7, 8.1, $1 \mathrm{H}, \mathrm{HC}(19))$; 5.60, 5.58, 5.54 (3 x s, 3 x 1 H, HC(30), HC35), $\mathrm{HC}(40)) ; 5.59(\mathrm{dd}, J=15.4,6.4,, 1 \mathrm{H}, \mathrm{HC}(18)) ; 4.48\left(\mathrm{AB}, J_{\mathrm{ab}}=10.7,2 \mathrm{H}\right.$, $\left.\mathrm{H}_{2} \mathrm{C}(45)\right) ; 4.44-4.30$ (m, 4 H, HC(3), HC(5), HC(9), HC(17)); 4.19 - 4.17 (m, 2 $\mathrm{H}, \mathrm{HC}(11), \mathrm{HC}(13)$ or $\mathrm{HC}(15)) ; 4.11-4.08(\mathrm{~m}, 1 \mathrm{H}, \mathrm{HC}(11)$ or $\mathrm{HC}(13)$ or $\mathrm{HC}(15)) ; 3.78\left(\mathrm{~s}, 3 \mathrm{H}, \mathrm{H}_{3} \mathrm{C}(50)\right) ; 3.17(\mathrm{~d}, J=3.9,1 \mathrm{H}, \mathrm{OH}) ; 2.98(\mathrm{t}, J=5.8$, $\mathrm{HC}(21)) ; 2.72\left(\mathrm{ABX}, J_{\mathrm{ax}}=7.5, J_{\mathrm{bx}}=5.2, J_{a b}=16.2,2 \mathrm{H}, \mathrm{H}_{2} \mathrm{C}(6)\right) ; 2.66\left(\mathrm{ABX}, J_{\mathrm{ax}}\right.$ $\left.=3.2, J_{\mathrm{bx}}=9.0, J_{\mathrm{ab}}=17.3,2 \mathrm{H}, \mathrm{H}_{2} \mathrm{C}(8)\right) 2.45(\mathrm{sext}, J=6.8,1 \mathrm{H}, \mathrm{HC}(20)) ; 2.13(\mathrm{~s}$, $2 \mathrm{H}, \mathrm{H}_{3} \mathrm{C}(25)$ ); 1.82 (sext, $J=6.5,1 \mathrm{H}, \mathrm{HC}(22)$ ); $1.82-1.67$ (m, $7 \mathrm{H}, \mathrm{H}_{2} \mathrm{C}(10)$, $\left.\mathrm{H}_{2} \mathrm{C}(14), \mathrm{H}_{\mathrm{eq}} \mathrm{C}(4), \mathrm{H}_{\mathrm{eq}} \mathrm{C}(12), \mathrm{H}_{\mathrm{eq}} \mathrm{C}(16)\right) ; 1.57$ - 1.43 (m, $3 \mathrm{H}, \mathrm{H}_{\mathrm{ax}} \mathrm{C}(4), \mathrm{H}_{\mathrm{ax}} \mathrm{C}(12)$, $\left.\mathrm{H}_{\mathrm{ax}} \mathrm{C}(16)\right) ; 1.06\left(\mathrm{~d}, J=6.6,3 \mathrm{H}, \mathrm{H}_{3} \mathrm{C}(51)\right) ; 0.95$ (d, $\left.J=6.3,3 \mathrm{H}, \mathrm{H}_{3} \mathrm{C}(23)\right) ; 0.94$ $\left(\mathrm{d}, J=6.6,3 \mathrm{H}, \mathrm{H}_{3} \mathrm{C}\left(23^{\prime}\right)\right)$ ) 0.04 (s, $6 \mathrm{H}, 2 \times \mathrm{H}_{3} \mathrm{C}(24)$ )

${ }^{13} \mathrm{C} \mathrm{NMR}: \quad\left(126 \mathrm{MHz}, \mathrm{CDCl}_{3}\right)$

208.27 (C(7)); 158.97 (C(49)); 145.55 (C(2)); 139.69 (C(26)); 138.73, 138.66, 138.06 (C(31), C(36), C(41)); 136.02 (C(19)); 131.12 (C(46)); 129.26 (2 x $\mathrm{C}(47))$; 128.85 (C(18)); 128.63, 128.58, 128.51, 128.19, 128.16, 128.14 (C(34), $\mathrm{C}(39), \mathrm{HC}(44), 2 \times \mathrm{C}(33), 2 \times \mathrm{C}(38), 2 \times \mathrm{C}(43), 2 \times \mathrm{C}(27), 2 \times \mathrm{C}(28), \mathrm{C}(1))$; 126.13, 126.11, 125.97 (2 x C(32), 2 x C(37), 2 x C(42)); 123.98 (C(29)); 113.62 (2 x C(48)); 100.62, 100.37 (C(30), C(35), C(40)); 88.33 (C(21)); 78.34 (C(3)); 77.24 (C(17)); 74.85 (C(45)); 73.50, 72.83, 72.55, 72.42 (C(5), C(11), C(13), $\mathrm{C}(15)) ; 64.15$ (C(9)); 55.21 (C(50)); 50.86 (C(8)); 49.13 (C(6)); 42.44, 42.04 (C(10), C(14)); 39.52 (C(20)); 37.57, 37.35, 36.25 (C(4), C(12), C(16)); 30.98 $(\mathrm{C}(22)) ; 25.80(\mathrm{C}(25)) ; 20.27,17.81\left(\mathrm{C}(23), \mathrm{C}\left(23^{\prime}\right)\right)$; $15.13(\mathrm{C}(51)) ;-3.57,-3.61$ $(2 \times \mathrm{C}(24))$ 
IR: $\quad\left(\mathrm{CH}_{2} \mathrm{Cl}_{2}\right)$

3522 (br, m); 3034 (m); 2955(s); 2914 (m); 2872 (m); 1712 (m); 1613 (m); 1514 (m); 1494 (m); 1453 (m); 1408 (m); 1338 (m); 1248 (m); 1212(m); 1174 (m); $1111(\mathrm{~m}) ; 1057(\mathrm{~m}) ; 1012(\mathrm{~m})$

MS: $\quad(F D)$

1024 (23); 1023 (54, M+); 1022 (77); 1021 (21); 931 (11); 122 (12); 121 (100); $106(14)$

Opt. Rot.: $\quad[\alpha]_{D}^{24}+45.3(\mathrm{c}=0.40, \mathrm{EtOH})$

TLC: $\quad R_{f} 0.30$ (hexane/EtOAc 1/1) [silica gel, $p$-anisaldehyde]

Analysis: $\quad$ Calc for $\mathrm{C}_{63} \mathrm{H}_{78} \mathrm{O}_{10} \mathrm{Si}(1023.37)$
Calcd:
C, 73.94\%;
$\mathrm{H}, 7.68 \%$
Found:
C, $73.92 \%$;
$\mathrm{H}, 7.86 \%$

\section{Determination of Configuration at C(9) in Aldol Adduct 15, (C(19) in RK-397).}

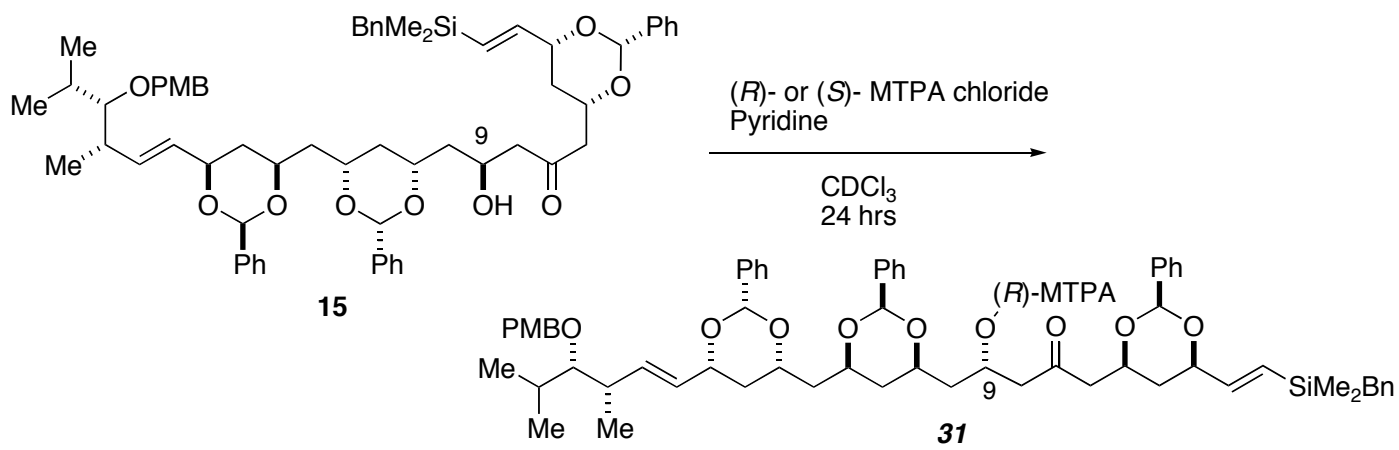

In an NMR tube was placed a solution of $15(4.1 \mathrm{mg}, 0.0039 \mathrm{mmol})$ in $\mathrm{CDCl}_{3}(0.75 \mathrm{~mL})$. To the solution was added sequentially pyridine $(0.02 \mathrm{~mL})$ and $(R)$ - or $(S)$ - $\alpha$-mehoxy- $\alpha$-trifluoromethylphenylacetyl chloride $(5.6 \mu \mathrm{L}, 0.031 \mathrm{mmol}, 8.0$ equiv). The reaction mixture was thoroughly mixed and was let stand for $24 \mathrm{~h}$ at rt. The crude Mosher ester 31 was analyzed by NMR and found to be of $S$ configuration. 
From (R)-MTPA-31

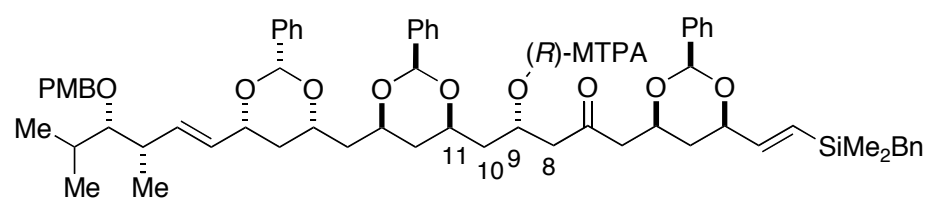

$$
\begin{aligned}
& \delta \mathrm{H}_{2} \mathrm{C}(8)=2.86 \mathrm{ppm} \\
& \delta \mathrm{H}_{2} \mathrm{C}(11)=3.80 \mathrm{ppm}
\end{aligned}
$$

\section{From $(S)$-MTPA-31}

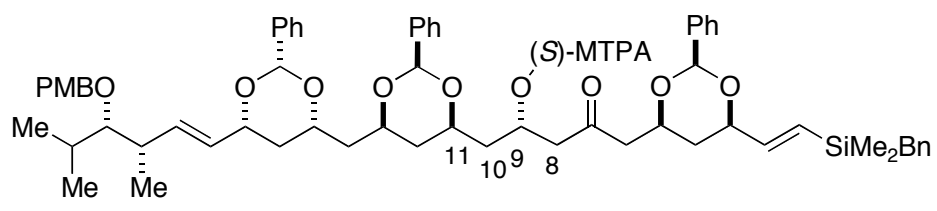

$$
\begin{aligned}
& \delta \mathrm{H}_{2} \mathrm{C}(8)=2.90 \mathrm{ppm} \\
& \delta \mathrm{H}_{2} \mathrm{C}(11)=3.62 \mathrm{ppm}
\end{aligned}
$$

Difference in chemical shifts:

$\Delta \delta_{S R} H_{2} \mathrm{C}(18)=+0.04 \mathrm{ppm}$

$\Delta \delta_{S R} \mathrm{HC}(21)=-0.18 \mathrm{ppm}$ 


\section{Acetonide 27}

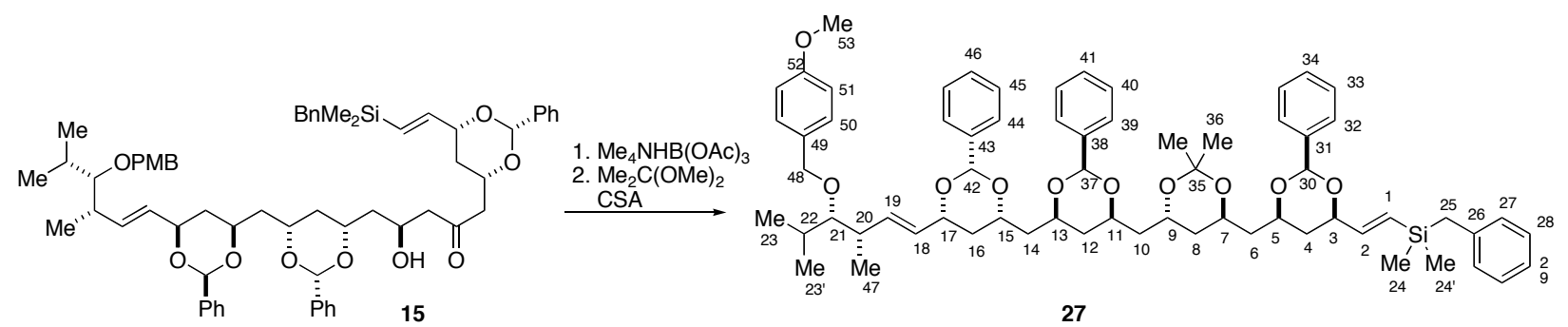

In a 100-mL, round-bottom flask were placed $15(1.34 \mathrm{~g}, 1.31 \mathrm{mmol})$, acetonitrile $(7 \mathrm{~mL})$ and acetic acid $(7 \mathrm{~mL})$. The solution was cooled to $0{ }^{\circ} \mathrm{C}$ (bath temperature) using an ice bath. To the solution was added tetramethylammonium triacetoxyborohydride $(1.72 \mathrm{~g}, 6.55 \mathrm{mmol}, 5.0$ equiv), and the reaction mixture was stirred at $0{ }^{\circ} \mathrm{C}$ for $10 \mathrm{~h}$. The reaction was quenched with saturated aqueous sodium potassium tartrate $(50 \mathrm{~mL})$ and the mixture was transferred to a 125 $\mathrm{mL}$ separatory funnel and then was extracted with dichloromethane $(2 \times 30 \mathrm{~mL})$. The combined organic extracts were washed with saturated aqueous $\mathrm{NaHCO}_{3}$ solution $(20 \mathrm{~mL})$ and brine $(20$ $\mathrm{mL}$ ), then was dried over $\mathrm{Na}_{2} \mathrm{SO}_{4}$ and concentrated under reduced pressure to afford a crude diol $(1.89 \mathrm{~g})$. In a $100-\mathrm{mL}$, round-bottom flask were placed the crude diol $(1.89 \mathrm{~g})$, dichloromethane (60 mL), 2,2-dimethoxypropane (1.59 mL, $13.1 \mathrm{mmol}, 10.0$ equiv) and $d l$-camphorsulfonic acid (5 mg). The solution was stirred at $\mathrm{rt}$ for $6 \mathrm{~h}$. The reaction was quenched with saturated aqueous $\mathrm{NaHCO}_{3}$ solution $(30 \mathrm{~mL})$ and the biphasic mixture was transferred to a $250-\mathrm{mL}$ separatory funnel. The aqueous layer was extracted with dichloromethane $(30 \mathrm{~mL})$ and the combined organic extracts were washed with saturated aqueous $\mathrm{NaHCO}_{3}$ solution $(30 \mathrm{~mL})$ and brine $(30 \mathrm{~mL})$. The extracts were dried over $\mathrm{Na}_{2} \mathrm{SO}_{4}$ and concentrated under reduced pressure. The resulting crude oil was chromatographed (silica gel, hexane/EtOAc $9 / 1$ to $5 / 1,50 \mathrm{~mm}$ ) to afford $1.21 \mathrm{~g}(1.14 \mathrm{mmol}, 87 \%)$ of 27 as white foam.

\section{Data for 27:}

mp: $\quad 61-62^{\circ} \mathrm{C}$

${ }^{1} \mathrm{H}$ NMR: $\quad\left(500 \mathrm{MHz}, \mathrm{CDCl}_{3}\right)$

$7.53-7.50$ (m, $6 \mathrm{H}, 2$ x $\mathrm{HC}(32), 2$ x HC(39), 2 x HC(44)); $7.39-7.30$ (m, 9 H, HC(34), 2 x HC(33), HC(41), 2 x HC(40), HC(46), 2 x HC(45)); 7.27 (d, J = 7.1, 
$2 \mathrm{H}, 2 \times \mathrm{HC}(50)) ; 7.20(\mathrm{t}, J=7.6,2 \mathrm{H}, 2 \times \mathrm{HC}(28)) ; 7.06(\mathrm{t}, J=7.5,1 \mathrm{H}$, $\mathrm{HC}(29)) ; 6.98$ (d, $J=7.1,2 \mathrm{H}, 2 \times \mathrm{HC}(27)) ; 6.83$ (d, $J=8.6,2 \mathrm{H}, 2 \times \mathrm{HC}(51)$; 6.08 (dd, $J=19.0,4.6,1 \mathrm{H}, \mathrm{HC}(2)) ; 5.92$ (dd, $J=18.8,1.2,1 \mathrm{H}, \mathrm{HC}(1)$ ); 5.77 (dd, $J=15.6,7.8,1 \mathrm{H}, \mathrm{HC}(19)) ; 5.60,5.57,5.56$ (3 x s, 3 x 1 H, HC(30), HC37), $\mathrm{HC}(42)) ; 5.56(\mathrm{dd}, J=18.4,6.5,1 \mathrm{H}, \mathrm{HC}(18)) ; 4.48\left(\mathrm{AB}, J_{\mathrm{ab}}=10.7,2 \mathrm{H}\right.$, $\left.\mathrm{H}_{2} \mathrm{C}(48)\right)$; 4.36 - 4.30 (m, $\left.2 \mathrm{H}, \mathrm{HC}(3), \mathrm{HC}(17)\right)$; 4.25 - 3.98 (m, $6 \mathrm{H}, \mathrm{HC}(5)$, $\mathrm{HC}(7), \mathrm{HC}(9), \mathrm{HC}(11), \mathrm{HC}(13), \mathrm{HC}(15)) ; 3.78$ (s, $\left.3 \mathrm{H}, \mathrm{H}_{3} \mathrm{C}(53)\right) ; 2.98$ (t, $J=5.7$, $\mathrm{HC}(21)$ ); 2.45 (sext, $J=6.3,1 \mathrm{H}, \mathrm{HC}(20)) ; 2.13$ (s, $2 \mathrm{H}, \mathrm{H}_{3} \mathrm{C}(25)$ ); 1.83 (sext, $J=$ 6.5, $1 \mathrm{H}, \mathrm{HC}(22)) ; 2.05$ - 1.99, 1.82 - 1.34 (m, $14 \mathrm{H}, \mathrm{H}_{2} \mathrm{C}(4), \mathrm{H}_{2} \mathrm{C}(6), \mathrm{HC}(8)$, $\left.\mathrm{H}_{2} \mathrm{C}(10), \mathrm{H}_{2} \mathrm{C}(12), \mathrm{H}_{2} \mathrm{C}(14), \mathrm{H}_{2} \mathrm{C}(16)\right) ; 1.38$ (s, $\left.3 \mathrm{H}, \mathrm{H}_{3} \mathrm{C}(36)\right) ; 1.37$ (s, $3 \mathrm{H}$, $\left.\mathrm{H}_{3} \mathrm{C}\left(36^{\prime}\right)\right) ; 1.07$ (d, $\left.J=6.6,3 \mathrm{H}, \mathrm{H}_{3} \mathrm{C}(47)\right) ; 0.95$ (d, $\left.J=6.6,3 \mathrm{H}, \mathrm{H}_{3} \mathrm{C}(23)\right) ; 0.94$ $\left(\mathrm{d}, J=6.8,3 \mathrm{H}, \mathrm{H}_{3} \mathrm{C}\left(23^{\prime}\right)\right)$; 0.05 (s, $6 \mathrm{H}, 2$ x $\left.\mathrm{H}_{3} \mathrm{C}(24)\right)$

${ }^{13} \mathrm{C} \mathrm{NMR}: \quad\left(126 \mathrm{MHz}, \mathrm{CDCl}_{3}\right)$

$158.98(\mathrm{C}(52)) ; 146.12(\mathrm{C}(2)) ; 139.76(\mathrm{C}(26)) ; 138.90,138.77,138.54(\mathrm{C}(31)$, C(38), C(43)); 136.01 (C(19)); 131.13 (C(49)); 129.26 (2 x C(50)); 128.87 (C(18)); 128.65, 128.58, 128.51, 128.21, 128.16, 128.05 (C(34), C(41), HC(46),2 x C(33), 2 x C(40), 2 x C(45), 2 x C(27), 2 x C(28), C(1)); 126.16, 126.14, 125.83 (2 x C(32), 2 x C(39), 2 x C(44)); 123.96 (C(29)); 113.96 (2 x C(51)); 100.48, 100.42, 100.37, 100.13 (C(30), C(35), C(37), C(42)); 88.31 (C(21)); 78..52 $(\mathrm{C}(3))$; 77.26 (C(17)); 74.83 (C(48)); 73.23, 73.04, 72.49 (C(5), C(11), C(13), $\mathrm{C}(15)) ; 62.84,62.38$ (C(7), C(9)); 55.17 (C(53)); 42.48, 41.82, 41.64 (C(6), $\mathrm{C}(10), \mathrm{C}(14))$; 39.49 (C(20)); 38.59, 37.74, 37.56, 36.24 (C(4), C(8), C(12), $\mathrm{C}(16)) ; 30.95$ (C(22)); 25.83 (C(25)); 24.68, 24.60 (2 x C(36)); 20.24, 17.79 $\left(\mathrm{C}(23), \mathrm{C}\left(23^{\prime}\right)\right) ; 15.10(\mathrm{C}(47)) ;-3.57,-3.63$ (2 x C(24))

IR: $\quad\left(\mathrm{CHCl}_{3}\right)$

3061 (w); 3025 (m); 2953(s); 2911 (m); 2869 (m); 1612 (m); 1514 (m); 1493 (m); 1452 (m); 1380 (m); 1341 (m); 1248 (m); 1224(m); 1119 (m); 1058 (m); 1028 (m); $1012(\mathrm{~m})$

MS: $\quad(H R$ ESI)

Calcd: 1087.5731 (for $\mathrm{C}_{66} \mathrm{H}_{84} \mathrm{O}_{10} \mathrm{Si}+\mathrm{Na}$ )

Found: 1087.5765 
Opt. Rot.: $\quad[\alpha]_{\mathrm{D}}^{24}+67.7(\mathrm{c}=0.60, \mathrm{EtOH})$

TLC: $\quad R_{f} 0.12$ (hexane/EtOAc, 5/1) [silica gel, $p$-anisaldehyde]

Analysis: $\quad$ Calc for $\mathrm{C}_{66} \mathrm{H}_{84} \mathrm{O}_{10} \mathrm{Si}(1065.45)$

$\begin{array}{lll}\text { Calcd: } & \text { C, } 74.40 \% ; & \text { H }, 7.95 \% \\ \text { Found: } & \text { C }, 74.18 \% ; & \text { H }, 7.86 \%\end{array}$

\section{Acetonide Aldehyde 28}
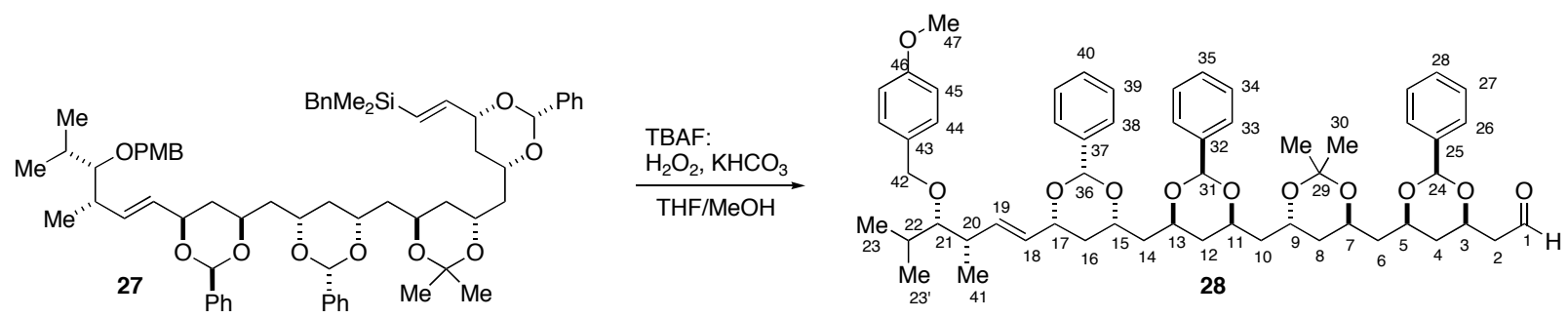

In a 10-mL Schlenk flask were placed $27(200 \mathrm{mg}, 019 \mathrm{mmol})$ and tetrahydrofuran (2 $\mathrm{mL}$ ). The solution was cooled to $0{ }^{\circ} \mathrm{C}$ (bath temperature) using an ice bath. To the solution was added dropwise a 1.0 M solution of TBAF in THF $(0.21 \mathrm{~mL}, 0.21 \mathrm{mmol}, 1.1$ equiv). The solution was stirred for $15 \mathrm{~min}$ at $0{ }^{\circ} \mathrm{C}$. To the solution was sequentially added $30 \% \mathrm{H}_{2} \mathrm{O}_{2}$ solution (0.36 mL, $4.51 \mathrm{mmol}, 24$ equiv), methanol (1.5 mL) and $\mathrm{KHCO}_{3}$ (94 mg, $0.94 \mathrm{mmol}, 5$ equiv). The reaction mixture was allowed to warm to $\mathrm{rt}$ and was stirred for $20 \mathrm{~h}$. To the mixture was added water $(4 \mathrm{~mL})$ and the resulting emulsion was stirred for $10 \mathrm{~min}$, then was diluted with ethyl acetate $(5 \mathrm{~mL})$ and transferred to a $60-\mathrm{mL}$ separatory funnel. The aqueous layer was extracted with ethyl acetate ( $2 \times 15 \mathrm{~mL})$ and the combined organic layers were washed with $30 \%$ aqueous $\mathrm{Na}_{2} \mathrm{~S}_{2} \mathrm{O}_{3}$ solution $(3 \times 15 \mathrm{~mL})$ and with brine $(10 \mathrm{~mL})$. The combined extracts were dried over $\mathrm{MgSO}_{4}$ and concentrated. The resulting clear oil was chromatographed (silica gel, hexanes/EtOAc, $30 \mathrm{~mm})$ to afford152 $\mathrm{mg}(0.16 \mathrm{mmol}, 86 \%)$ of $\mathbf{2 8}$ as white foam.

\section{Data for 28:}

mp: $\quad 77-78^{\circ} \mathrm{C}$

${ }^{1}$ H NMR: $\quad\left(500 \mathrm{MHz}, \mathrm{CDCl}_{3}\right)$

$9.86(\mathrm{t}, J=1.8,1 \mathrm{H}, \mathrm{HC}(1)) ; 7.53-7.45(\mathrm{~m}, 6 \mathrm{H}, 2$ x $\mathrm{HC}(26), 2$ x $\mathrm{HC}(33), 2$ x 
$\mathrm{HC}(38)) ; 7.39$ - 7.31 (m, 9 H, HC(28), 2 x HC(27), HC(35), 2 x HC(34), HC(40), 2 x $\mathrm{HC}(39)) ; 7.27$ (d, $J=9.0,2 \mathrm{H}, 2 \times \mathrm{HC}(44)) ; 6.84$ (d, $J=8.6,2 \mathrm{H}, 2$ x $\mathrm{HC}(45) ; 5.77$ (dd, 15.8, 8.0, $1 \mathrm{H}, \mathrm{HC}(19)) ; 5.60,5.57,5.56$ (3 x s, 3 x $1 \mathrm{H}$, $\mathrm{HC}(24), \mathrm{HC}(31), \mathrm{HC}(36)) ; 5.61-5.53$ (m, $1 \mathrm{H}, \mathrm{HC}(18)) ; 4.49$ (AB, $J_{\mathrm{ab}}=10.8,2$ $\left.\mathrm{H}, \mathrm{H}_{2} \mathrm{C}(42)\right) ; 4.42$ - 4.37 (m, $\left.1 \mathrm{H}, \mathrm{HC}(3)\right) ; 4.37$ - 4.33 (m, $1 \mathrm{H}, \mathrm{HC}(17)$ ); 4.26 3.99 (m, $6 \mathrm{H}, \mathrm{HC}(5), \mathrm{HC}(7), \mathrm{HC}(9), \mathrm{HC}(11), \mathrm{HC}(13), \mathrm{HC}(15)) ; 3.78$ (s, $3 \mathrm{H}$, $\left.\mathrm{H}_{3} \mathrm{C}(47)\right) ; 2.98(\mathrm{t}, J=5.8, \mathrm{HC}(21)) ; 2.72\left(\mathrm{ABXd}, J_{\mathrm{ax}}=7.4, J_{\mathrm{bx}}=5.1, J_{\mathrm{ab}}=16.9, \mathrm{~J}\right.$ $=2.3,2 \mathrm{H}, \mathrm{H}_{2} \mathrm{C}(2)$ ); 2.45 (sext, $J=6.7,1 \mathrm{H}, \mathrm{HC}(20)$ ); 1.83 (sext, $J=8.5,1 \mathrm{H}$, $\mathrm{HC}(22)) ; 2.04-1.97,1.82-1.39$ (m, $14 \mathrm{H}, \mathrm{H}_{2} \mathrm{C}(4), \mathrm{H}_{2} \mathrm{C}(6), \mathrm{HC}(8), \mathrm{H}_{2} \mathrm{C}(10)$, $\left.\mathrm{H}_{2} \mathrm{C}(12), \mathrm{H}_{2} \mathrm{C}(14), \mathrm{H}_{2} \mathrm{C}(16)\right) ; 1.37$ (s, $3 \mathrm{H}, \mathrm{H}_{3} \mathrm{C}(30)$ ); 1.36 (s, $3 \mathrm{H}, \mathrm{H}_{3} \mathrm{C}(30$ ')); 1.07 (d, $\left.J=6.6,3 \mathrm{H}, \mathrm{H}_{3} \mathrm{C}(41)\right) ; 0.95$ (d, $\left.J=6.6,3 \mathrm{H}, \mathrm{H}_{3} \mathrm{C}(23)\right) ; 0.94$ (d, $J=6.9,3$ $\left.\mathrm{H}, \mathrm{H}_{3} \mathrm{C}\left(23^{\prime}\right)\right)$

${ }^{13} \mathrm{C} \mathrm{NMR}: \quad\left(126 \mathrm{MHz}, \mathrm{CDCl}_{3}\right)$

200.39 (C(1)); 159.02 (C(46)); 138.92, 138.80, 138.17 (C(25), C(32), C(37)); 136.06 (C(19)); 131.18 (C(43)); 129.30 (2 x C(44)); 128.90 (C(18)); 128.79, 128.62, 128.55 (C(28), C(35), C(40)); 128.22, 128.19 (2 x C(27), 2 x C(34), 2 x $\mathrm{C}(39)) ; 126.19,125.98,125.86$ ( 2 x C(26), 2 x C(33), 2 x C(38)); $113.66(2 \times$ $\mathrm{C}(45)) ; 100.71,100.45,100.16$ (C(24), C(29), C(31), C(36)); 88.36 (C(21)); 77.26 (C(17)); 74.87 (C(42)); 73.29, 73.05, 72.53, 72.52 (C(5), C(11), C(13), $\mathrm{C}(15)) ; 71.85(\mathrm{C}(3)) ; 62.79,62.40(\mathrm{C}(7), \mathrm{C}(9)) ; 55.22$ (C(47)); $49.34(\mathrm{C}(2))$; 42.51, 41.82, 41.50 (C(6), C(10), C(14)); 39.54 (C(20)); 38.61, 37.76, 37.60, 36.16 (C(4), C(8), C(12), C(16)); 30.98 (C(22)); 24.65, 24.59 (2 x C(30)); 20.27, $17.79\left(\mathrm{C}(23), \mathrm{C}\left(23^{\prime}\right)\right) ; 15.14(\mathrm{C}(41))$

IR: $\quad\left(\mathrm{CHCl}_{3}\right)$

3061 (w); 3025 (m); 2953(s); 2911 (m); 2869 (m); 1612 (m); 1514 (m); 1493 (m); 1452 (m); 1380 (m); 1341 (m); 1248 (m); 1224(m); 1119 (m); 1058 (m); 1028 (m); $1012(\mathrm{~m})$

MS: $\quad(\mathrm{FD})$

934 (10); 933 (31); $932\left(40, \mathrm{M}^{+}\right) ; 904$ (10); 122 (26); 121 (100)

Opt. Rot.: $\quad[\alpha]_{\mathrm{D}}^{24}+44.1(\mathrm{c}=0.45, \mathrm{EtOH})$

TLC: $\quad R_{f} 0.20$ (hexane/EtOAc 2/1) [silica gel, $p$-anisaldehyde] 
Analysis: $\quad$ Calc for $\mathrm{C}_{57} \mathrm{H}_{72} \mathrm{O}_{11}(933.17)$
Calcd:
C, $73.36 \%$;
H, $7.78 \%$
Found:
C, $73.15 \%$;
H, $7.86 \%$

\section{Hydroxy Aldehyde 16}

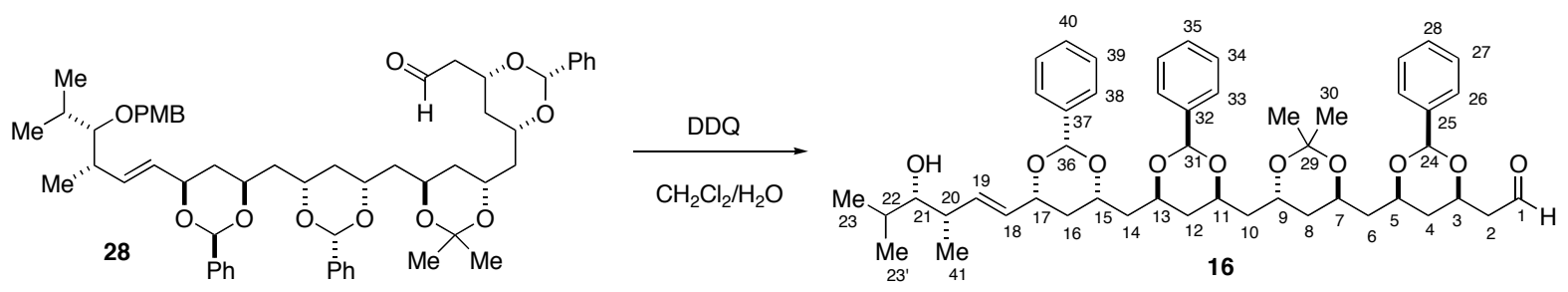

In a 100-mL Schlenk flask were placed 28 (136 mg, $0.15 \mathrm{mmol})$, dichloromethane (7 $\mathrm{mL}$ ) and water $(0.35 \mathrm{~mL})$. To the solution was added DDQ (36 mg, $0.16 \mathrm{mmol}, 1.1 \mathrm{equiv})$ in one portion and the reaction mixture was stirred for $25 \mathrm{~min}$ at $\mathrm{rt}$. To the reaction mixture was added another portion of DDQ ( $5 \mathrm{mg}, 0.02 \mathrm{mmol}, 0.1$ equiv) and the mixture was stirred for an additional $25 \mathrm{~min}$ at $\mathrm{rt}$. To the reaction mixture was added saturated aqueous $\mathrm{NaHCO}_{3}$ solution $(8 \mathrm{~mL})$. The biphasic mixture was stirred for $15 \mathrm{~min}$ and was transferred to a $60-\mathrm{mL}$ separatory funnel. The aqueous layer was extracted with dichloromethane $(2 \times 15 \mathrm{~mL})$ and the combined organic layer was washed with brine $(10 \mathrm{~mL})$. The organic extracts dried over $\mathrm{Na}_{2} \mathrm{SO}_{4}$ and concentrated under reduced pressure. The resulting crude oil was chromatographed (silica gel, hexane/EtOAc 3/1 to 1/1, $20 \mathrm{~mm})$ to afford $107 \mathrm{mg}(0.13 \mathrm{mmol}, 90 \%)$ of $\mathbf{1 6}$ as white foam.

\section{Data for 16:}

mp: $\quad 74-75^{\circ} \mathrm{C}$

${ }^{1} \mathrm{H} \mathrm{NMR}: \quad\left(500 \mathrm{MHz}, \mathrm{CDCl}_{3}\right)$

9.86 (s, $1 \mathrm{H}, \mathrm{HC}(1)) ; 7.55-7.46$ (m, $6 \mathrm{H}, 2$ x $\mathrm{HC}(26), 2$ x $\mathrm{HC}(33), 2 \times \mathrm{HC}(38))$;

$7.40-7.33(\mathrm{~m}, 9 \mathrm{H}, \mathrm{HC}(28), 2$ x $\mathrm{HC}(27), \mathrm{HC}(35), 2$ x $\mathrm{HC}(34), \mathrm{HC}(40), 2$ x $\mathrm{HC}(39)) ; 5.73(\mathrm{dd}, J=15.7,7.3,1 \mathrm{H}, \mathrm{HC}(19)) ; 5.63(\mathrm{dd}, J=15.5,5.8,1 \mathrm{H}$, $\mathrm{HC}(18)$ ); 5.62, 5.58, 5.57 (3 x s, 3 x 1 H, HC(24), HC(31), HC(36)); 4.43 - 4.36 (m, $2 \mathrm{H}, \mathrm{HC}(3), \mathrm{HC}(17)) ; 4.28-4.02$ (m, $6 \mathrm{H}, \mathrm{HC}(5), \mathrm{HC}(7), \mathrm{HC}(9), \mathrm{HC}(11)$, $\mathrm{HC}(13), \mathrm{HC}(15)) ; 3.76(\mathrm{t}, J=6.3,1 \mathrm{H}, \mathrm{OH}) ; 3.17(\mathrm{td}, J=5.3,4.9,1 \mathrm{H}, \mathrm{HC}(21))$; 
$2.72\left(\mathrm{ABXd}, J_{\mathrm{ax}}=7.4, J_{\mathrm{bx}}=4.2, J_{\mathrm{ab}}=16.9, \mathrm{~J}=2.0,2 \mathrm{H}, \mathrm{H}_{2} \mathrm{C}(2)\right) ; 2.38(\mathrm{sext}, J=$ 6.5, $1 \mathrm{H}, \mathrm{HC}(20)) ; 2.04-1.97,1.86$ - 1.39 (m, $15 \mathrm{H}, \mathrm{HC}(22), \mathrm{H}_{2} \mathrm{C}(4), \mathrm{H}_{2} \mathrm{C}(6)$, $\mathrm{HC}(8), \mathrm{H}_{2} \mathrm{C}(10), \mathrm{H}_{2} \mathrm{C}(12), \mathrm{H}_{2} \mathrm{C}(14), \mathrm{H}_{2} \mathrm{C}(16)$ ); 1.39 (s, $3 \mathrm{H}, \mathrm{H}_{3} \mathrm{C}(30)$ ); 1.37 (s, 3 $\mathrm{H}, \mathrm{H}_{3} \mathrm{C}\left(30\right.$ ')); 1.04 (d, $\left.J=6.6,3 \mathrm{H}, \mathrm{H}_{3} \mathrm{C}(41)\right)$; 0.93 (d, $J=7.3,3 \mathrm{H}, \mathrm{H}_{3} \mathrm{C}(23)$ ); $0.92\left(\mathrm{~d}, J=7.5,3 \mathrm{H}, \mathrm{H}_{3} \mathrm{C}\left(23^{\prime}\right)\right)$

${ }^{13} \mathrm{C} \mathrm{NMR}: \quad\left(126 \mathrm{MHz}, \mathrm{CDCl}_{3}\right)$

200.37 (C(1)); 138.88, 138.67, 138.14 (C(25), C(32), C(37)); 135.07 (C(19)); $130.00(\mathrm{C}(18))$; 128.75, 128.64, 128.52 (C(28), C(35), C(40)); 128.18, 128.16 (2 x C(27), 2 x C(34), 2 x C(39)); 126.16, 125.95, 125.83 (2 x C(26), 2 x C(33), 2 x $\mathrm{C}(38)$ ); 100.66, 100.47, 100.40, 100.12 (C(24), C(29), C(31), C(36)); 79.56 $(\mathrm{C}(21)) ; 77.13$ (C(17)); 73.26, 73.01, 72.49 (C(5), C(11), C(13), C(15)); 71.81 $(\mathrm{C}(3)) ; 62.76,62.36(\mathrm{C}(7), \mathrm{C}(9)) ; 49.29(\mathrm{C}(2))$; 42.45, 41.79, 41.46 (C(6), C(10), $\mathrm{C}(14))$; 39.35 (C(20)); 38.57, 37.71, 37.63, 36.12 (C(4), C(8), C(12), C(16)); 30.47 (C(22)); 24.62, 24.55 (2 x C(30)); 19.62, 16.96 (C(23), C(23’)); 13.92 (C(41))

IR: $\quad\left(\mathrm{CH}_{2} \mathrm{Cl}_{2}\right)$

3584 (br, w); 3053 (w); 2952(s); 1726(m); 1458 (m); 1381 (m); 1343 (m); 1265 (s); $1224(\mathrm{~m}) ; 1113(\mathrm{~m}) ; 1059(\mathrm{~m}) ; 1011$ (m)

MS: $\quad$ (FD)

814 (22); 813 (45); $812\left(55, \mathrm{M}^{+}\right) ; 811$ (15); 799 (22); 798 (55); 797 (100); 740 (33); 739 (49)

Opt. Rot.: $\quad[\alpha]_{\mathrm{D}}^{24}+29.7(\mathrm{c}=0.17, \mathrm{EtOH})$

TLC: $\quad R_{f} 0.28$ (hexane/EtOAc, $\left.1 / 1\right)$ [silica gel, $p$-anisaldehyde]

Analysis: $\quad$ Calc for $\mathrm{C}_{49} \mathrm{H}_{64} \mathrm{O}_{10}(813.03)$

Calcd: $\quad$ C, $72.39 \% ; \quad H, 7.93 \%$

Found: $\quad \mathrm{C}, 72.18 \% ; \quad \mathrm{H}, 8.01 \%$ 


\section{1-Benzyldimethylsilyl-7-(tetrahydropyan-2-yloxy)-1,3,5-heptatriene (19)}
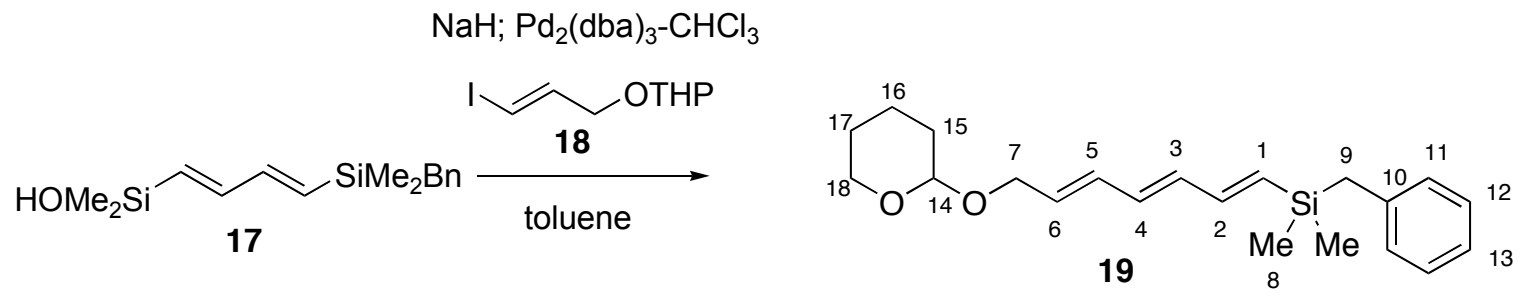

To a 10-mL Schlenk flask were placed washed sodium hydride (40 mg, 1.69 mmol, 1.4 equiv) and toluene $(0.5 \mathrm{~mL})$. To this suspension was added solution of 17 (501 $\mathrm{mg}, 1.81 \mathrm{mmol}$, 1.5 equiv) in toluene $(2.5 \mathrm{~mL})$ dropwise over $5 \mathrm{~min}$, and the reaction mixture was stirred for 15 min at rt. To the solution was sequentially added 18 (328 $\mathrm{mg}, 1.21 \mathrm{mmol})$ and tris(dibenzylideneacetone)dipalladium(0) chloroform adduct (62.6 mg, $0.061 \mathrm{mmol}, 0.05$ equiv). The reaction mixture was stirred for $6 \mathrm{~h}$ and then was passed through a pad of silica gel (60 mm x $2 \mathrm{~cm})$. The silica gel was washed with ethyl acetate $(3 \times 20 \mathrm{~mL})$, and the filtrate was concentrated under reduced pressure. The crude oil was chromatographed (silica gel, hexane/EtOAc 9/1, $30 \mathrm{~mm})$ to afford $319 \mathrm{mg}(0.93 \mathrm{mmol}, 77 \%)$ of 19 as a yellow oil. An analytically pure sample was obtained by further chromatography (silica gel, hexanes/ether 15/1, $30 \mathrm{~mm}$ ) followed by bulb-to-bulb distillation (ABT $165^{\circ} \mathrm{C}$ at $0.1 \mathrm{mmHg}$ ).

\section{Data for 19:}

bp: $\quad 165^{\circ} \mathrm{C}(\mathrm{ABT}, 0.1 \mathrm{mmHg})$

${ }^{1} \mathrm{H} \mathrm{NMR}: \quad\left(500 \mathrm{MHz}, \mathrm{CDCl}_{3}\right)$

$7.21(\mathrm{t}, J=7.6,2 \mathrm{H}, 2 \times \mathrm{HC}(12)) ; 7.07$ (t, $J=7.3,1 \mathrm{H}, \mathrm{HC}(13)) ; 6.99$ (d, $J=7.3$, $2 \mathrm{H}, 2$ x HC(11))); $6.54-6.49$ (m, $1 \mathrm{H}, \mathrm{HC}(2))$; 6.32 - 6.27 (m, 1 H, HC(3)); 6.24 - 6.22 (m, 2 H, HC(4), HC(5));5.87 - 5.82 (m, 2 H, HC(1), HC(6)); 4.66 (t, J = 3.6, $1 \mathrm{H}, \mathrm{HC}(14)) ; 4.18\left(\mathrm{ABX}, J_{\mathrm{ab}}=13.4, J_{\mathrm{ax}}=5.9, J_{\mathrm{bx}}=6.8,2 \mathrm{H}, \mathrm{H}_{2} \mathrm{C}(7)\right) ; 3.90-$ 3.86 (m, $\left.1 \mathrm{H}, \mathrm{H}_{\mathrm{eq}} \mathrm{C}(18)\right)$; 3.54-3.50 (m, $\left.1 \mathrm{H}, \mathrm{H}_{\mathrm{ax}} \mathrm{C}(18)\right)$; 2.15 (s, $\left.2 \mathrm{H}, \mathrm{H}_{2} \mathrm{C}(9)\right)$ ) 1.88 - 1.82 (m, $\left.1 \mathrm{H}, \mathrm{H}_{\mathrm{eq}} \mathrm{C}(15)\right) ; 1.76-1.72$ (m, $\left.1 \mathrm{H}, \mathrm{H}_{\mathrm{ax}} \mathrm{C}(15)\right) ; 1.64-1.53$ (m, $4 \mathrm{H}$, $\left.\mathrm{H}_{2} \mathrm{C}(16), \mathrm{H}_{2} \mathrm{C}(17)\right)$; 0.06 (s, $\left.6 \mathrm{H}, 2 \mathrm{x}_{3} \mathrm{C}(8)\right)$ 
${ }^{13} \mathrm{C} \mathrm{NMR}: \quad\left(126 \mathrm{MHz}, \mathrm{CDCl}_{3}\right)$

$144.88(\mathrm{C}(2)) ; 13987(\mathrm{C}(10)) ; 135.40$ (C(4)); 132.79 (C(5)); 132.60 (C(1)); 132.33 (C(3)); 130.79 (C(6)); 128.23 (2 x C(11)); 128.11 (2 x C(12)); 123.97 (C(13)); 97.87 (C(14)); 67.24 (C(7)); 62.18 (C(18)); 30.56 (C(15)); 26.00 (C(17)); $25.40(\mathrm{C}(9)) ; 19.40(\mathrm{C}(16)) ;-3.47(2 \times \mathrm{C}(8))$

IR: (neat)

$3081(\mathrm{w}) ; 3059(\mathrm{~m}) ; 3024(\mathrm{~m}) ; 2947(\mathrm{~s}) ; 1727(\mathrm{w}) ; 1686(\mathrm{~m}) ; 1642(\mathrm{w}) ; 1600(\mathrm{~m})$; 1560 (m); 1493 (m); 1452 (m); 1441 (m); 1406 (m); 1350 (m); 1322 (m); 1284 (m); 1248 (m); 1203 (m); 1183 (m); 1155 (m); 1119 (m); 1078 (m); 1055 (m); $1024(\mathrm{~s}) ; 1006$ (s)

MS: $\quad(\mathrm{EI}, 70 \mathrm{eV})$

$342\left(2, \mathrm{M}^{+}\right) ; 251$ (11); 149 (22); 91 (12); 85 (100); 75 (29); 59 (11); 57 (17)

TLC: $\quad R_{f} 0.05$ (hexane/EtOAc, 9/1) [silica gel, $p$-anisaldehyde]

Analysis: $\quad \mathrm{C}_{21} \mathrm{H}_{30} \mathrm{O}_{2} \mathrm{Si}(342.55)$

Calcd: $\quad$ C, $73.63 \% ; \quad \mathrm{H}, 8.83 \%$

Found: $\quad$ C, $73.62 \% ; \quad H, 9.11 \%$

\section{Ethyl 10-(Tetrahydropyran-2-yl)-2,4,6,8-tetraenoate (20)}

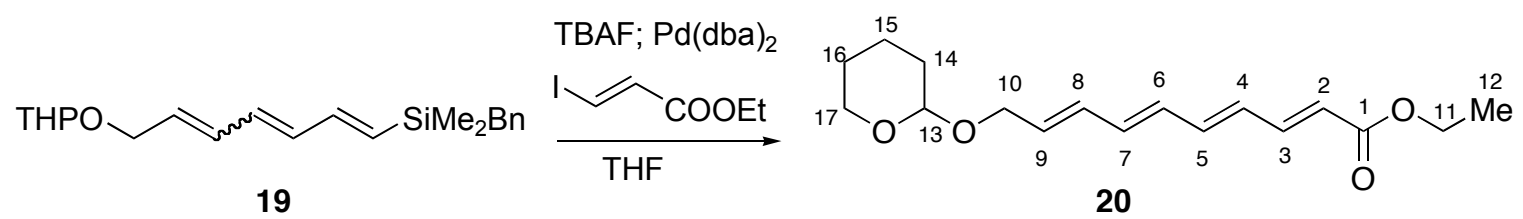

In a 10-mL Schlenk flask was placed 19 (360 mg, $1.05 \mathrm{mmol}, 1.2$ equiv) and tetrahydrofuran $(3 \mathrm{~mL})$. The solution was cooled to $0{ }^{\circ} \mathrm{C}$ (bath temperature) using an ice bath. To the solution was added dropwise a 1.0 M solution of TBAF in THF (2.10 mL, $2.10 \mathrm{mmol}, 2.4$ equiv) and the solution was stirred for $15 \mathrm{~min}$ at $0{ }^{\circ} \mathrm{C}$. To the solution was added sequentially ethyl 3-iodoacrylate (198 mg, $0.88 \mathrm{mmol}$ ) and bis(dibenzylieneacetone)palladium(0) (23.1 mg, $0.044 \mathrm{mmol}, 0.05$ equiv). The reaction mixture was allowed to warm to $\mathrm{rt}$ and was stirred for 7 h. The reaction mixture was passed through a pad of silica gel $(60 \mathrm{~mm} \times 2 \mathrm{~cm})$ and the silica gel was washed with ethyl acetate $(3 \times 20 \mathrm{~mL})$. The filtrate was concentrated under reduced 
pressure. The resulting crude oil was chromatographed (silica gel, hexanes/EtOAc 7/1, $30 \mathrm{~mm}$ ) to afford 20 (204 mg, $0.70 \mathrm{mmol}, 79 \%)$ as yellow powder. An analytically pure sample was obtained by recrystallization of $\mathbf{2 0}(60 \mathrm{mg})$ from refluxing hexanes/ether 3/1 (20 mL).

Data for 20:

mp: $\quad 59-60^{\circ} \mathrm{C}$

${ }^{1} \mathrm{H}$ NMR: $\quad\left(500 \mathrm{MHz}, \mathrm{CDCl}_{3}\right)$

$7.31(\mathrm{dd}, J=15.3,11.4,1 \mathrm{H}, \mathrm{HC}(3)) ; 6.58(\mathrm{dd}, J=14.7,11.0,1 \mathrm{H}, \mathrm{HC}(5))$; 6.44 6.27 (m, 4 H, HC(4), HC(6), HC(7), HC(8)); 5.92 (dt, $J=14.4,6.0, \mathrm{HC}(9)) ; 5.87$ $(\mathrm{d}, J=15.1,1 \mathrm{H}, \mathrm{HC}(2)) ; 4.65(\mathrm{t}, J=3.7,1 \mathrm{H}, \mathrm{HC}(13)) ; 4.19\left(\mathrm{ABX}, J_{\mathrm{ab}}=13.9, J_{\mathrm{ax}}\right.$ $\left.=6.0, J_{\mathrm{bx}}=6.6,2 \mathrm{H}, \mathrm{H}_{2} \mathrm{C}(10)\right) ; 4.20\left(\mathrm{q}, J=7.1,2 \mathrm{H}, \mathrm{H}_{2} \mathrm{C}(11)\right) ; 3.89-3.85(\mathrm{~m}, 1$ $\left.\mathrm{H}, \mathrm{H}_{\mathrm{eq}} \mathrm{C}(17)\right) ; 3.54-3.50\left(\mathrm{~m}, 1 \mathrm{H}, \mathrm{H}_{\mathrm{ax}} \mathrm{C}(17)\right) ; 1.86-1.82\left(\mathrm{~m}, 1 \mathrm{H}, \mathrm{H}_{\mathrm{eq}} \mathrm{C}(14)\right)$; $1.77-1.71$ (m, $\left.1 \mathrm{H}, \mathrm{H}_{\mathrm{ax}} \mathrm{C}(14)\right) ; 1.65-1.52$ (m, $\left.4 \mathrm{H}, \mathrm{H}_{2} \mathrm{C}(15), \mathrm{H}_{2} \mathrm{C}(16)\right) ; 1.30$ (t, $J$ $\left.=7.1,3 \mathrm{H}, \mathrm{H}_{3} \mathrm{C}(12)\right)$

${ }^{13} \mathrm{C} \mathrm{NMR}: \quad\left(126 \mathrm{MHz}, \mathrm{CDCl}_{3}\right)$

167.11 (C(1)); 144.28 (C(3)); 140.44 (C(5)); 136.28 (C(4)); 132.54 (C(6)); 131.86 (C(9)); 131.82 (C(7)); 130.16 (C(8)); 120.81 (C(2)); 97.97 (C(13)); 67.07 (C(10)); 62.19 (C(17)); $60.25(\mathrm{C}(11)) ; 30.53(\mathrm{C}(14))$; 25.37(C(15) or C(16)); 19.34 (C(15) or $\mathrm{C}(16)) ; 14.27(\mathrm{C}(12))$

IR: (neat)

2944(s); $2872(\mathrm{~m}) ; 1702(\mathrm{~s}) ; 1654(\mathrm{~m}) ; 1624(\mathrm{~m}) ; 1599(\mathrm{~m}) ; 1466(\mathrm{~m}) ; 1368(\mathrm{~m})$; 1340 (m); 1302 (m); 1261 (m); 1201 (m); 1127 (m); 1077 (m); 1008 (m)

MS: $\quad(\mathrm{EI}, 70 \mathrm{eV})$

292 (0.3, M+); 208 (19); 191 (11); 117 (24); 91 (12); 85 (100); 67 (13); 57 (17)

TLC: $\quad R_{f} 0.08$ (hexane/EtOAc, 7/1) [silica gel, $p$-anisaldehyde]

Analysis: $\quad \mathrm{C}_{17} \mathrm{H}_{24} \mathrm{O}_{4}(292.37)$

Calcd: $\quad$ C, $69.84 \% ; \quad \mathrm{H}, 8.27 \%$

Found: $\quad$ C, $69.73 \%$; H, $8.33 \%$ 


\section{Ethyl Pentaenoate 21}

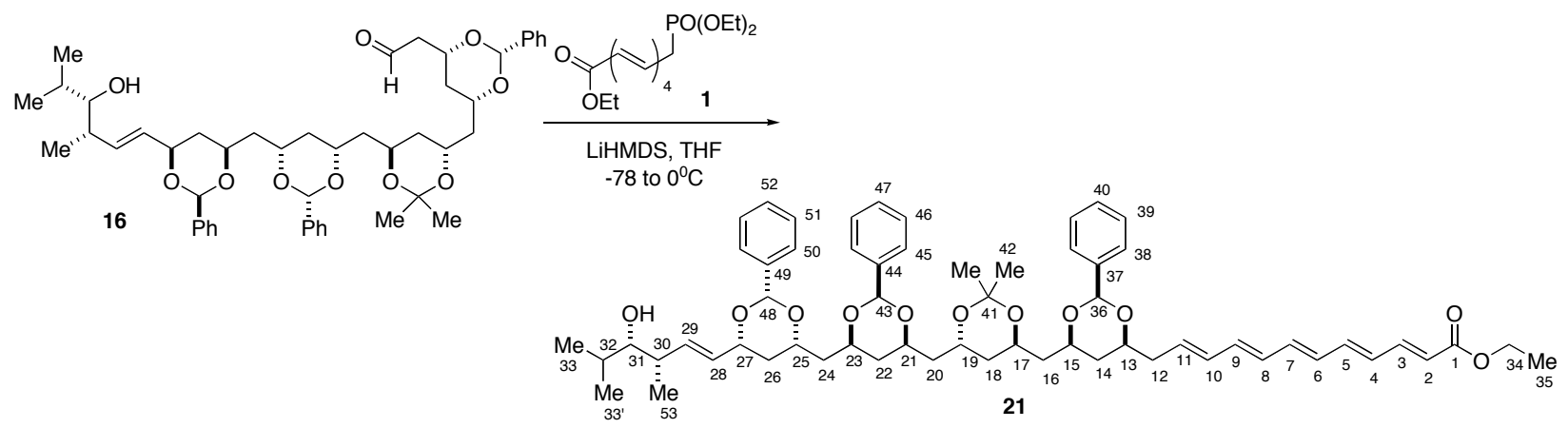

In a 50-mL Schlenk flask was placed 1 (59 $\mathrm{mg}, 0.18 \mathrm{mmol}, 2.5$ equiv) and tetrahydrofuran $(15 \mathrm{~mL})$. The solution was cooled to $-72{ }^{\circ} \mathrm{C}$ (internal temperature) using a dry ice/isopropanol bath. To the solution was added dropwise a $0.2 \mathrm{M}$ solution of LiHMDS in THF (0.9 mL, $0.18 \mathrm{mmol}, 2.5$ equiv). The dark blue solution was stirred for $15 \mathrm{~min}$ before the addition of a solution of $16(58 \mathrm{mg}, 0.072 \mathrm{mmol})$ in tetrahydrofuran $(5 \mathrm{~mL})$. The reaction mixture was stirred at $-72{ }^{\circ} \mathrm{C}$ for $15 \mathrm{~min}$, then was allowed to warm to $0{ }^{\circ} \mathrm{C}$ using an ice bath and was stirred for $45 \mathrm{~min}$ at $0{ }^{\circ} \mathrm{C}$. The reaction mixture was quenched with saturated aqueous $\mathrm{NaHCO}_{3}$ solution $(20 \mathrm{~mL})$. The biphasic mixture was transferred to a $125-\mathrm{mL}$ separatory funnel. The aqueous layer was extracted with ether $(2 \times 20 \mathrm{~mL})$ and the combined organic layers were washed with brine $(20 \mathrm{~mL})$. The extracts were dried over $\mathrm{Na}_{2} \mathrm{SO}_{4}$ and then were concentrated under reduced pressure. The resulting crude oil was chromatographed (silica gel, hexanes/EtOAc 3/1, $20 \mathrm{~mm})$ to afford $54.7 \mathrm{mg}(0.055 \mathrm{mmol}, 77 \%)$ of 21 as yellow solid.

\section{Data for 21:}

$\underline{\mathrm{mp}}: \quad 88-90^{\circ} \mathrm{C}$

${ }^{1} \mathrm{H}$ NMR: $\quad\left(500 \mathrm{MHz}, \mathrm{C}_{6} \mathrm{D}_{6}\right)$

$7.75-7.69$ (m, $6 \mathrm{H}, 2 \times \mathrm{HC}(38), 2 \times \mathrm{HC}(45), 2 \times \mathrm{HC}(50)) ; 7.52$ (dd, $J=15.2$, 11.5, 1H, HC(3)); 7.23 - 7.10 (m, 9 H, HC(40), 2 x HC(39), HC(47), 2 x HC(46), $\mathrm{HC}(52), 2$ x HC(51); 6.23 - 5.93 (m, 8 H, HC(2), HC(4), HC(5), HC(6), HC(7), $\mathrm{HC}(8), \mathrm{HC}(9), \mathrm{HC}(10)) ; 5.83$ (dt, $J=14.5,7.4,1 \mathrm{H}, \mathrm{HC}(11)) ; 5.65$ (dd, $J=15.6$, 7.6, $1 \mathrm{H}, \mathrm{HC}(29)) ; 5.57$ (dd, $J=15.7,5.2,1 \mathrm{H}, \mathrm{HC}(28)) ; 5.54,5.54,5.45$ (3 x s, 3 
x $1 \mathrm{H}, \mathrm{HC}(36), \mathrm{HC}(43), \mathrm{HC}(48)) ; 4.09$ (q, $J=7.0,2 \mathrm{H}, \mathrm{H}_{2} \mathrm{C}(34)$ ); 4.33 - 4.27, 4.28 - $3.86(\mathrm{~m}, 7 \mathrm{H}, \mathrm{HC}(15), \mathrm{HC}(17), \mathrm{HC}(19), \mathrm{HC}(21), \mathrm{HC}(23), \mathrm{HC}(25)$, $\mathrm{HC}(27)) ; 3.61-3.56$ (m, $1 \mathrm{H}, \mathrm{HC}(13)) ; 2.95$ (m, $1 \mathrm{H}, \mathrm{HC}(31)) ; 2.46-2.25(\mathrm{~m}, 2$ $\mathrm{H}, \mathrm{H}_{2} \mathrm{C}(12)$ ); 2.23 (sext, $J=7.1,1 \mathrm{H}, \mathrm{HC}(30)$ ); $2.13-2.07,1.72-1.10$ (m, $15 \mathrm{H}$, $\left.\mathrm{HC}(32), \mathrm{H}_{2} \mathrm{C}(14), \mathrm{H}_{2} \mathrm{C}(16), \mathrm{HC}(18), \mathrm{H}_{2} \mathrm{C}(20), \mathrm{H}_{2} \mathrm{C}(22), \mathrm{H}_{2} \mathrm{C}(24), \mathrm{H}_{2} \mathrm{C}(26)\right) ; 1.46$ (s, $\left.3 \mathrm{H}, \mathrm{H}_{3} \mathrm{C}(30)\right)$ ) 1.42 (s, $\left.3 \mathrm{H}, \mathrm{H}_{3} \mathrm{C}\left(30^{\prime}\right)\right) ; 1.07$ (d, $\left.J=5.7,1 \mathrm{H}, \mathrm{OH}\right) ; 1.02$ (d, $J=$ 7.1, $\left.3 \mathrm{H}, \mathrm{H}_{3} \mathrm{C}(53)\right) ; 1.02\left(\mathrm{t}, J=7.1,3 \mathrm{H}, \mathrm{H}_{3} \mathrm{C}(35)\right) ; 0.91(\mathrm{~d}, J=6.9,3 \mathrm{H}$, $\left.\mathrm{H}_{3} \mathrm{C}(33)\right) ; 0.80\left(\mathrm{~d}, J=6.8,3 \mathrm{H}, \mathrm{H}_{3} \mathrm{C}\left(33^{\prime}\right)\right)$

${ }^{13} \mathrm{C} \mathrm{NMR}: \quad\left(126 \mathrm{MHz}, \mathrm{C}_{6} \mathrm{D}_{6}\right)$

$166.76(\mathrm{C}(1))$; $144.58(\mathrm{C}(3))$; $140.76(\mathrm{C}(11)) ; 140.05,139.85,139.69(\mathrm{C}(37)$, $\mathrm{C}(44), \mathrm{C}(49))$; 137.23, 135.53, 133.33, 132.07, 132.04, 131.55, 130.15 (C(4), $\mathrm{C}(5), \mathrm{C}(6), \mathrm{C}(7), \mathrm{C}(8), \mathrm{C}(9), \mathrm{C}(10)) ; 134.79$ (C(29)); 130.36 (C(28)); 128.86, 128.81, 128.29, 126.94, 126.73, 126.71 (C(40), C(47), C(52), 2 x C(39), 2 x $\mathrm{C}(46), 2$ x C(51), 2 x C(38), 2 x C(45), 2 x C(50)); 121.15 (C(2)); 101.25, 101.22, 100.42, 99.29 (C(36), C(41), C(43), C(48)); 79.47 (C(31)); 77.10 (C(27)); 76.37 $(\mathrm{C}(13)) ; 73.60,73.26,72.70,72.62$ (C(15), C(21), C(23), C25)); 63.13, 62.63 (C(17), C(19)); 60.13 (C(34)); 43.21, 42.52, 42.22 (C(16), C(20), C(24)); 40.16 (C(30)); 39.83 (C(12)); 38.99, 38.19, 38.03, 36.40 (C(14), C(18), C(22), C(26)); 30.85 (C(32)); 25.00, 24.94 (2 x C(42)); 19.88, 16.70 (C(33), C(33’)); 14.95 $(\mathrm{C}(53)) ; 14.31(\mathrm{C}(35))$

IR: $\quad\left(\mathrm{CH}_{2} \mathrm{Cl}_{2}\right)$

3524 (br, w); 3019 (m); 2982 (m); 2947(s); 2916 (m); 2872 (m); 1706(s); 1622 (m); 1579 (m); 1454 (m); 1429 (w); 1408 (m); 1378 (m); 1343 (m); 1309 (m); $1224(\mathrm{~m}) ; 1128(\mathrm{~s}) ; 1058(\mathrm{~m}) ; 1011$ (s)

MS: $\quad(H R-E S I)$

Calcd: 1009.5442 (for $\mathrm{C}_{61} \mathrm{H}_{78} \mathrm{O}_{11}+\mathrm{Na}$ )

Found: 1009.5452

Opt. Rot.: $\quad[\alpha]_{D}^{24}+19.0(\mathrm{c}=0.05, \mathrm{EtOH})$

TLC: $\quad R_{f} 0.48$ (hexane/EtOAc, $\left.1 / 1\right)$ [silica gel, $p$-anisaldehyde] 


\section{Macrolactone 29}

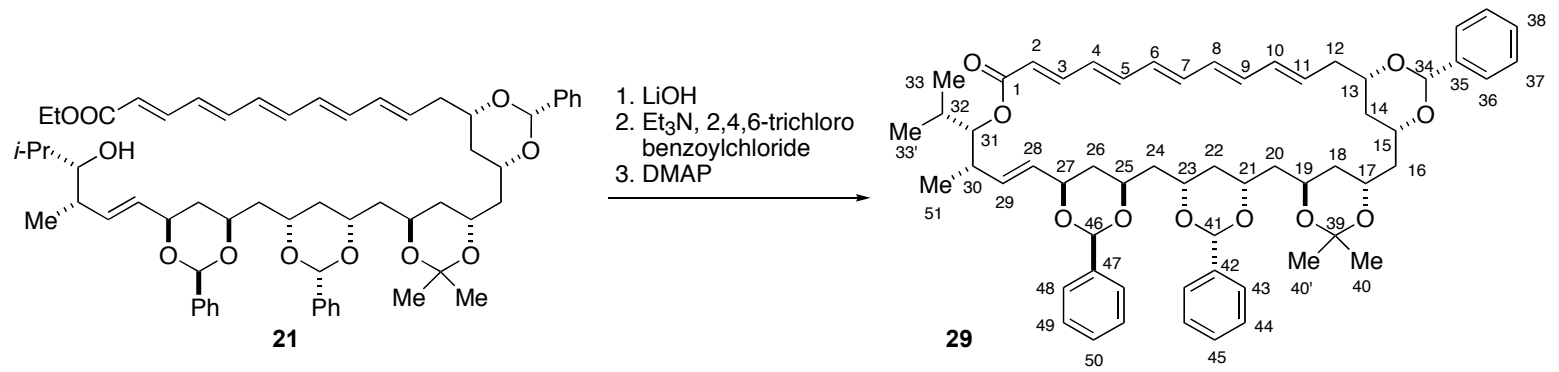

In a 25-mL, round-bottom flask was placed sequentially 21 (14 mg, 0.014 mmol), tetrahydrofuran $(2 \mathrm{~mL})$, methanol $(0.5 \mathrm{~mL})$, water $(0.5 \mathrm{~mL})$ and a $1.0 \mathrm{M}$ aqueous $\mathrm{LiOH}(1.0 \mathrm{~mL}$, $1.0 \mathrm{mmol}$ ). The solution was stirred for $16 \mathrm{~h}$ at $\mathrm{rt}$, then was quenched by the addition of $1 \mathrm{~mL}$ of water. The reaction mixture was transferred to a $60-\mathrm{mL}$ separatory funnel and extracted with ethyl acetate $(3 \times 15 \mathrm{~mL})$. The combined extracts were washed with brine $(15 \mathrm{~mL})$, dried over $\mathrm{Na}_{2} \mathrm{SO}_{4}$ and concentrated under reduced pressure to afford the crude acid as pale yellow oil. The crude acid was placed in a 100-mL Schlenk flask together with a $0.04 \mathrm{M}$ solution of triethylamine in THF(3.5 mL, $0.14 \mathrm{mmol}, 10.0$ equiv) followed by $0.04 \mathrm{M}$ solution of 2,4,6trichlorobenzoyl chloride in THF (2.45 $\mathrm{mL}, 0.10 \mathrm{mmol}, 7.0$ equiv), and the reaction mixture was stirred at $\mathrm{rt}$ for $1 \mathrm{~h}$. The volatile materials were removed under vacuum $(0.5 \mathrm{mmHg})$ and the residue was dissolved in toluene $(50 \mathrm{~mL})$. In a $250-\mathrm{mL}$, 3-neck flask were placed 4dimethylaminopyridine $(30 \mathrm{mg})$ and toluene $(125 \mathrm{~mL})$, to which was added dropwise over $8 \mathrm{~h}$ the solution of the activated anhydride in toluene using a cannula. The resulting reaction mixture was stirred for additional $12 \mathrm{~h}$. The reaction was quenched with saturated aqueous $\mathrm{NaHCO}_{3}$ solution $(20 \mathrm{~mL})$ and was transferred to a $500-\mathrm{mL}$ separatory funnel. The aqueous layer was extracted with ether $(100 \mathrm{~mL})$ and the combined organic extracts were washed with brine $(50$ $\mathrm{mL}$ ). The extracts were dried over $\mathrm{Na}_{2} \mathrm{SO}_{4}$ and concentrated under reduced pressure. The resulting crude oil was chromatographed (silica gel, hexanes/EtOAc 4/1, $20 \mathrm{~mm}$ ) to afford 9.3 $\mathrm{mg}(0.010 \mathrm{mmol}, \mathbf{7 1 \%})$ of $\mathbf{2 9}$ as pale yellow powder. 


\section{Data for 29:}

mp: $\quad 83-85^{\circ} \mathrm{C}($ dec. $)$

${ }^{1} \mathrm{H}$ NMR: $\quad\left(500 \mathrm{MHz}, \mathrm{C}_{6} \mathrm{D}_{6}\right)$

$7.78-7.64$ (m, $6 \mathrm{H}, 2 \times \mathrm{HC}(36), 2 \times \mathrm{HC}(43), 2 \times \mathrm{HC}(48)) ; 7.60$ (dd, $J=15.2$, 10.8, 1H, HC(3)); 7.26 - 7.10 (m, 9 H, HC(38), 2 x HC(37), HC(45), 2 x HC(44), $\mathrm{HC}(50), 2$ x HC(49)); 6.10 - 5.90 (m, 8 H, HC(2), HC(4), HC(5), HC(6), HC(7), $\mathrm{HC}(8), \mathrm{HC}(9), \mathrm{HC}(10)) ; 5.56$ - 5.36 (m, 3 H, HC(29), HC(28), HC(11)); 5.53, 5.46, 5.46 (3 x s, 3 x $1 \mathrm{H}, \mathrm{HC}(34), \mathrm{HC}(41), \mathrm{HC}(46))$; 5.09 (dd, J = 9.8, 3.0, $1 \mathrm{H}$, $\mathrm{HC}(31)) ; 4.19$ - 3.79 (m, 7 H, HC(15), HC(17), HC(19), HC(21), HC(23), $\mathrm{HC}(25), \mathrm{HC}(27)) ; 3.52$ - 3.48 (m, $1 \mathrm{H}, \mathrm{HC}(13)) ; 2.65$ - 2.62 (m, $\left.1 \mathrm{H}, \mathrm{H}_{2} \mathrm{C}(12)\right)$; $2.52-2.49$ (m, $1 \mathrm{H}, \mathrm{HC}(30)) ; 2.30-2.21$ (m, $\left.1 \mathrm{H}, \mathrm{H}_{2} \mathrm{C}(12)\right) ; 1.72-0.92$ (m, 15 $\left.\mathrm{H}, \mathrm{HC}(32), \mathrm{H}_{2} \mathrm{C}(14), \mathrm{H}_{2} \mathrm{C}(16), \mathrm{HC}(18), \mathrm{H}_{2} \mathrm{C}(20), \mathrm{H}_{2} \mathrm{C}(22), \mathrm{H}_{2} \mathrm{C}(24), \mathrm{H}_{2} \mathrm{C}(26)\right)$; 1.40 (s, $3 \mathrm{H}, \mathrm{H}_{3} \mathrm{C}(30)$ ); 1.39 (s, $3 \mathrm{H}, \mathrm{H}_{3} \mathrm{C}\left(30\right.$ ’)); 1.50 (d, $J=7.1,3 \mathrm{H}, \mathrm{H}_{3} \mathrm{C}(53)$ ); $1.01\left(\mathrm{~d}, J=6.6,3 \mathrm{H}, \mathrm{H}_{3} \mathrm{C}(33)\right) ; 0.70$ (d, $\left.J=6.8,3 \mathrm{H}, \mathrm{H}_{3} \mathrm{C}\left(33^{\prime}\right)\right)$

${ }^{13} \mathrm{C} \mathrm{NMR}: \quad\left(126 \mathrm{MHz}, \mathrm{C}_{6} \mathrm{D}_{6}\right)$

$166.80(\mathrm{C}(1))$; $145.59(\mathrm{C}(3))$; 141.09 (C(11)); 140.14, 140.05, 139.69 (C(35), C(42), C(47)); 137.84, 137.18, 135.01, 134.65, 132.07, 132.02, 131.67, 130.72, 130.62 (C(4), C(5), C(6), C(7), C(8), C(9), C(10), C(29), C(28)); 129.96, 129.87, 129.28, 128.81, 128.76, 128.51, 127.50, 127.19, 126.77, 126.75, 125.65 (C(38), C(45), C(50), 2 x C(37), 2 x C(44), 2 x C(49), 2 x C(36), 2 x C(43), 2 × C(48)); $121.25(\mathrm{C}(2))$; 101.34, 101.29, 100.91, 100.10 (C(34), C(39), C(41), C(46)); 80.07 (C(31)); 75.56, 75.24, 74.48, 73.33, 71.91, 71.86 (C(13), C(15), C(21), $\mathrm{C}(23), \mathrm{C} 25), \mathrm{C}(27)) ; 63.22,61.12$ (C(17), C(19)); 44.16, 43.58, 41.89 (C(16), $\mathrm{C}(20), \mathrm{C}(24)) ; 39.57$ (C(12)); 36.52 (C(30)); 40.00, 39.22, 35.67 (C(14), C(18), $\mathrm{C}(22), \mathrm{C}(26)) ; 29.75(\mathrm{C}(32)) ; 25.79,25.61$ (2 x C(40)); 20.01, 18.69 (C(33), $\left.\mathrm{C}\left(33^{\prime}\right)\right) ; 11.05(\mathrm{C}(51))$

IR: $\quad\left(\mathrm{CH}_{2} \mathrm{Cl}_{2}\right)$

2916 (s); 1702 (m); 1619 (m); 1578 (m); 1379 (m); 1344 (m); 1224 (m); 1125 (s); $1010(\mathrm{~s})$ 
MS: $\quad(\mathrm{HR}-\mathrm{ESI})$

Calcd: 963.5023 (for $\mathrm{C}_{59} \mathrm{H}_{72} \mathrm{O}_{10}+\mathrm{Na}$ )

Found: 963.5028

Opt. Rot.: $\quad[\alpha]_{\mathrm{D}}^{24}+44.9(\mathrm{c}=0.085, \mathrm{EtOH})$

TLC: $\quad R_{f} 0.55$ (hexane/EtOAc, $\left.1 / 1\right)$ [silica gel, $p$-anisaldehyde]

\section{RK-397}
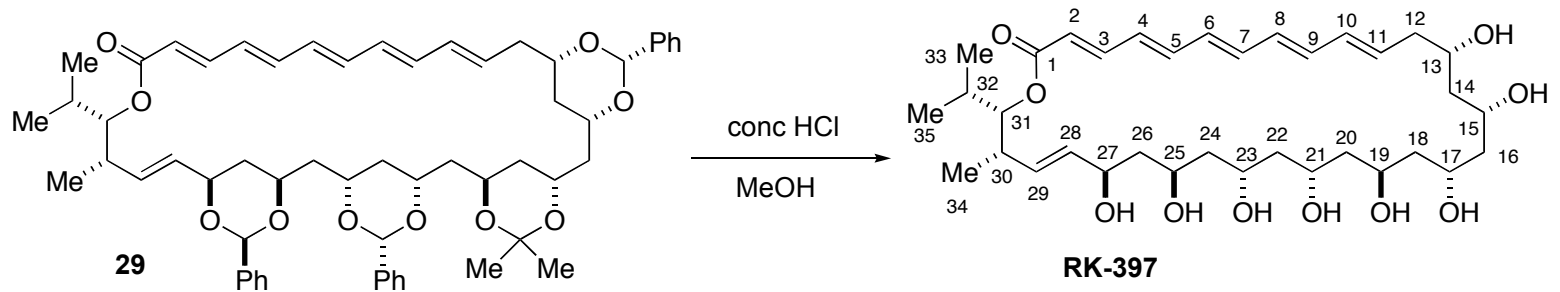

In a $25-\mathrm{mL}$, round-bottom flask were placed methanol $(2 \mathrm{~mL})$ and conc. $\mathrm{HCl}(0.05 \mathrm{~mL}$, $0.6 \mathrm{mmol})$. To the solution was added dropwise a solution of $29(9.3 \mathrm{mg}, 0.010 \mathrm{mmol})$ in methanol $(5 \mathrm{~mL})$. The reaction solution was stirred for $5 \mathrm{~h}$, then was neutralized using polymerbound piperidine (400 mg, ca. $1.2 \mathrm{mmol}$ ). The polymer was removed by filtration and the filtrate was concentrated under reduced pressure. The resulting crude solid was chromatographed (silica gel, EtOAc/MeOH 4/1, $10 \mathrm{~mm}$ ) to afford RK-397 and partially deprotected macrolactone. The partially deprotected macrolactone was resubjected under the reaction conditions described above to afford ca. $7 \mathrm{mg}$ of RK-397 combined. After silica gel chromatography, the synthetic RK-397 was further purified by reversed-phase preparative HPLC (Vydac C-18 250 x 22 mm, $60 / 40$ to $80 / 20 \mathrm{MeOH} / \mathrm{H}_{2} \mathrm{O}$ over $45 \mathrm{~min}$, flow $8 \mathrm{~mL} / \mathrm{min}$, $\mathrm{UV}$ detector at $360 \mathrm{~nm}$; retention time $43 \mathrm{~min}$ ). The HPLC purification was repeated twice to obtain pure synthetic RK-397 (6.0 mg, $0.0094 \mathrm{mmol}, 93 \%$ ) as yellow powder. 


\section{Comparison of Natural and Synthetic RK-397:}
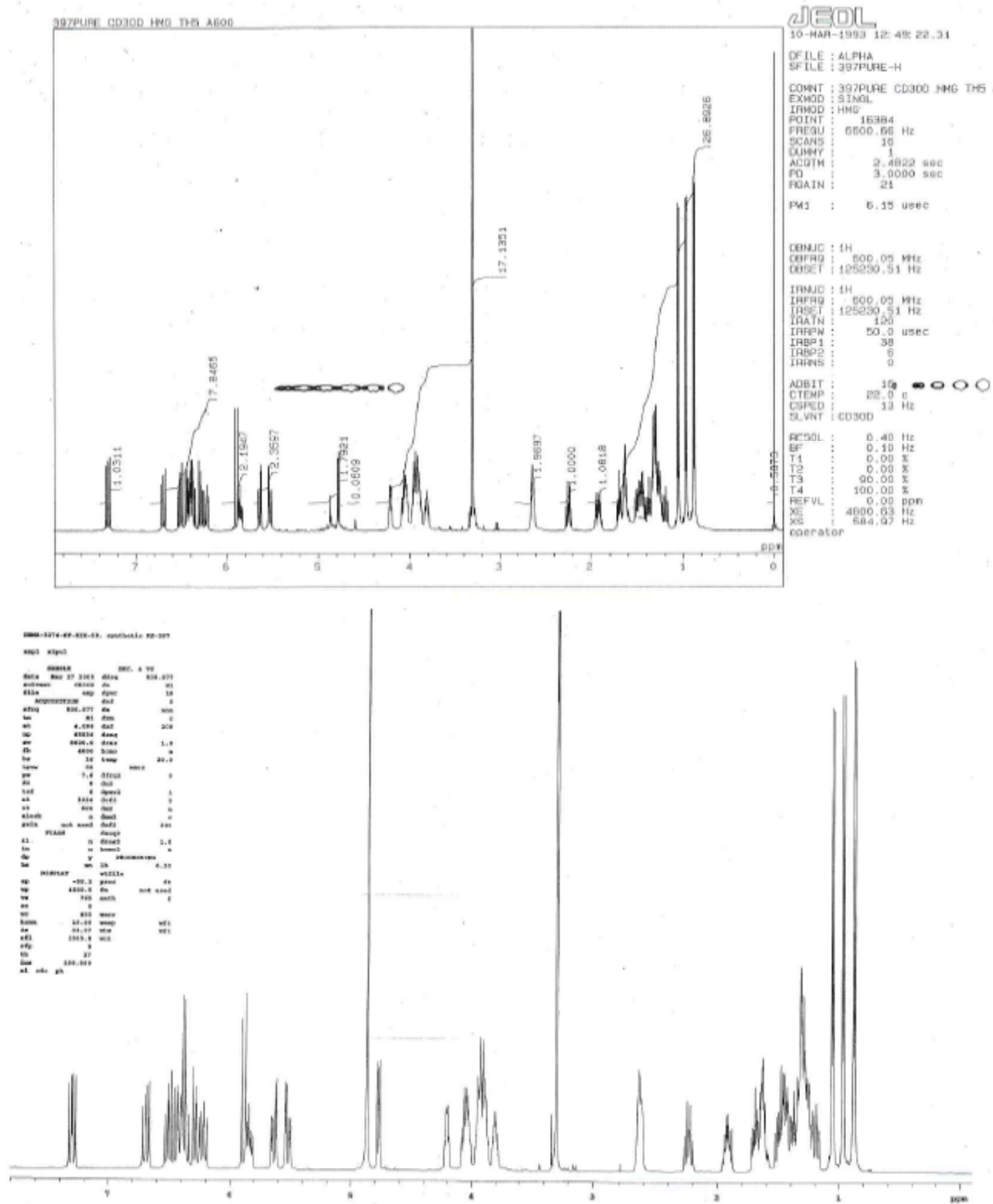

Figure 1. ${ }^{1} \mathrm{H}$ NMR spectra of RK-397 (top: natural RK-397 from RIKEN; bottom: synthetic RK-397 from UIUC) 

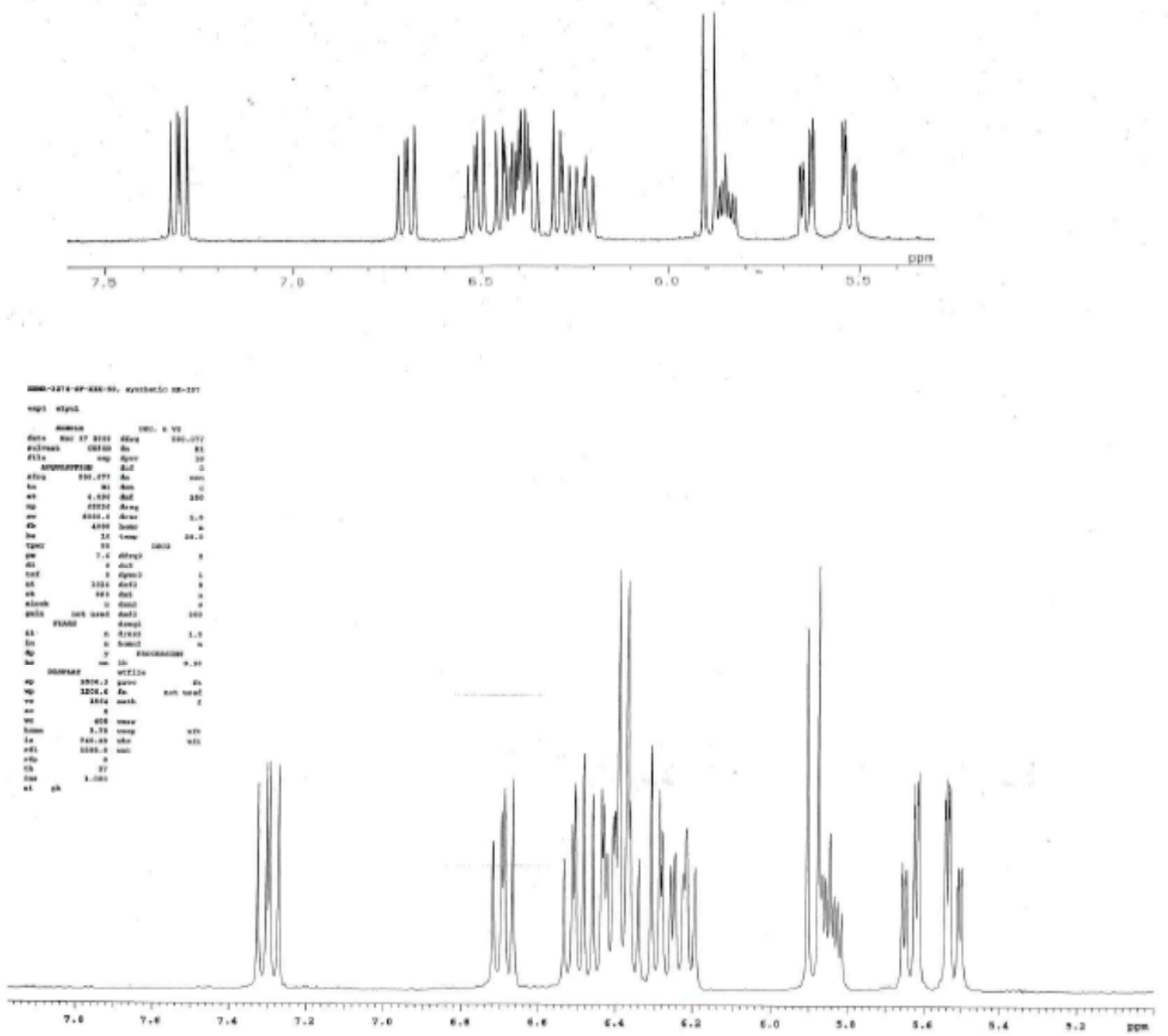

Figure 2. ${ }^{1} \mathrm{H}$ NMR spectra of RK-397: 5.2 - 7.9 ppm (top: natural RK-397 from RIKEN; bottom: synthetic RK-397 from UIUC) 

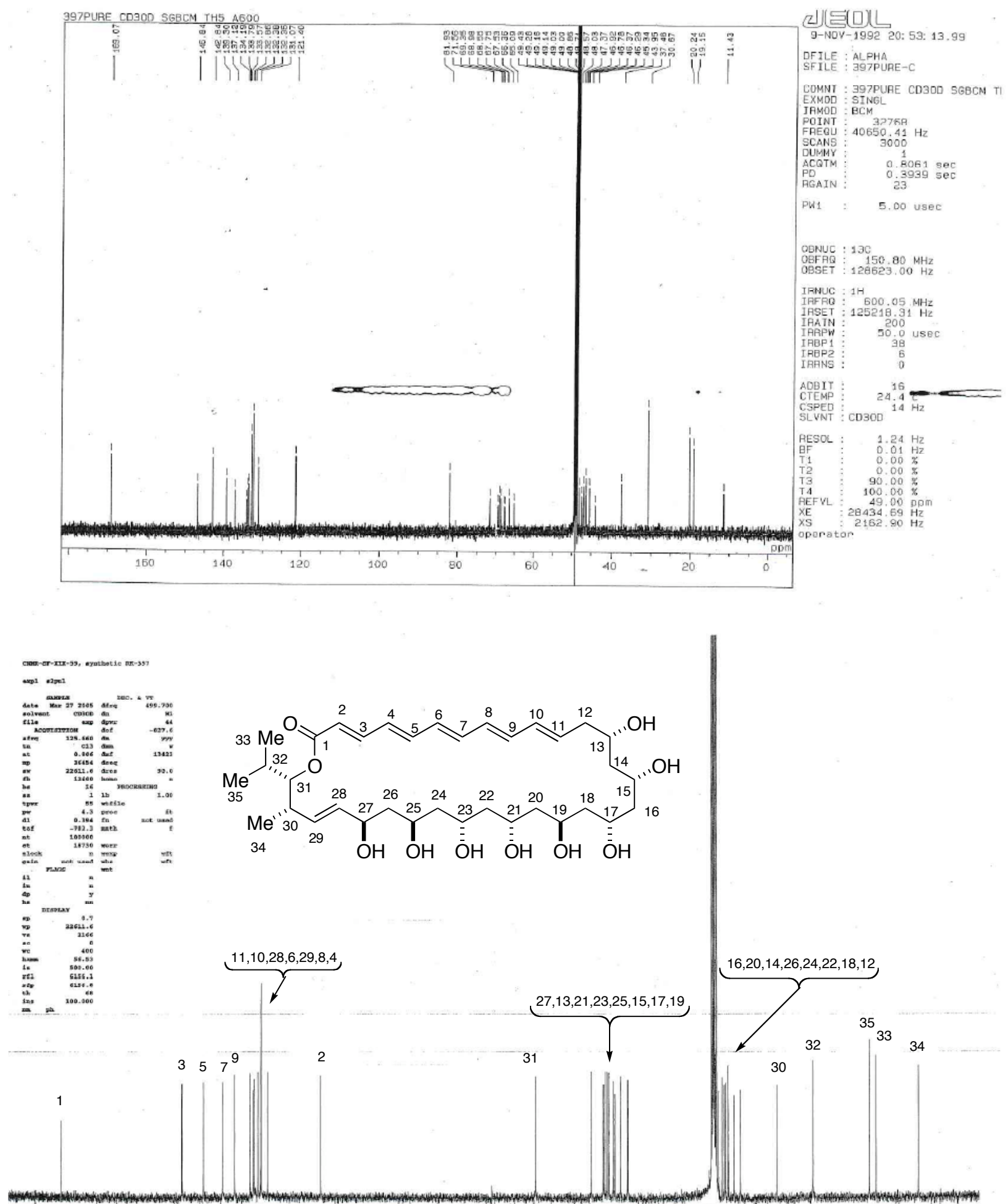

Figure 3. ${ }^{13} \mathrm{C}$ spectra of RK-397 (top: natural RK-397 from RIKEN; bottom: synthetic RK-397 from UIUC) 
I. NMR Spectroscopic Properties:

${ }^{13} \mathrm{CNMR}$ (Natural at $150 \mathrm{MHz}$; Synthetic at $125 \mathrm{MHz}$ )

\begin{tabular}{|c|c|c|c|}
\hline Carbon No. & Natural (ppm) & Synthetic (ppm) & Difference (ppm) \\
\hline 1 & 169.07 & 169.10 & +0.03 \\
\hline 2 & 121.40 & 121.44 & +0.04 \\
\hline 3 & 146.84 & 146.90 & +0.06 \\
\hline 4 & 131.07 & 131.16 & +0.09 \\
\hline 5 & 142.84 & 142.89 & +0.15 \\
\hline 6 & 132.86 & 132.92 & +0.06 \\
\hline 7 & 139.30 & 139.37 & +0.07 \\
\hline 8 & 132.38 & 132.38 & 0 \\
\hline 9 & 137.12 & 137.20 & +0.08 \\
\hline 10 & 133.79 & 133.77 & -0.02 \\
\hline 11 & 134.19 & 134.31 & +0.12 \\
\hline 12 & 43.95 & 44.11 & +0.16 \\
\hline 13 & 69.35 & 69.22 & -0.13 \\
\hline 14 & 46.92 & 47.14 & +0.22 \\
\hline 15 & 67.53 & 67.34 & -0.19 \\
\hline 16 & 48.03 & 48.11 & +0.08 \\
\hline 17 & 66.36 & 66.25 & -0.11 \\
\hline 18 & 45.34 & 45.32 & -0.02 \\
\hline 19 & 65.09 & 64.94 & -0.15 \\
\hline 20 & 47.37 & 47.50 & +0.13 \\
\hline 21 & 68.98 & 68.87 & -0.11 \\
\hline 22 & 46.29 & 46.32 & +0.03 \\
\hline 23 & 68.55 & 68.45 & -0.10 \\
\hline 24 & 46.37 & 46.36 & -0.01 \\
\hline 25 & 67.75 & 67.61 & -0.14 \\
\hline 26 & 46.78 & 46.86 & +0.08 \\
\hline 27 & 71.56 & 71.46 & -0.10 \\
\hline 28 & 133.57 & 133.55 & -0.02 \\
\hline 29 & 132.35 & 132.38 & +0.03 \\
\hline 30 & 37.48 & 37.43 & -0.05 \\
\hline 31 & 81.93 & 81.88 & -0.05 \\
\hline 32 & 30.67 & 30.70 & +0.03 \\
\hline 33 & 19.15 & 19.11 & -0.04 \\
\hline 34 & 11.43 & 11.34 & -0.09 \\
\hline 35 & 20.24 & 20.27 & +0.03 \\
\hline
\end{tabular}


${ }^{1} \mathrm{HNMR}$ (Natural at $600 \mathrm{MHz}$; Synthetic at $500 \mathrm{MHz}$ )

\begin{tabular}{|c|c|c|}
\hline Carbon No. & Natural (ppm) & Synthetic (ppm) \\
\hline 1 & -- & -- \\
\hline 2 & $5.89(\mathrm{~d}, 14.9)$ & $5.89(\mathrm{~d}, 15.1)$ \\
\hline 3 & $7.30(\mathrm{dd}, 14.9,11.7)$ & $7.30(\mathrm{dd}, 14.9,11.5)$ \\
\hline 4 & $6.44(\mathrm{dd}, 14.9,11.7)$ & $6.43(\mathrm{dd}, 14.7,11.6)$ \\
\hline 5 & $6.70(\mathrm{dd}, 14.9,11.3)$ & $6.69(\mathrm{dd}, 14.6,11.0)$ \\
\hline 6 & $6.37(\mathrm{dd}, 14.9,11.3)$ & $6.37(\mathrm{dd}, 15.2,11.0)$ \\
\hline 7 & $6.51(\mathrm{dd}, 14.9,10.9)$ & $6.51(\mathrm{dd}, 14.7,11.0)$ \\
\hline 8 & $6.29(\mathrm{dd}, 14.9,10.9)$ & $6.28(\mathrm{dd}, 14.8,10.9)$ \\
\hline 9 & $6.40(\mathrm{dd}, 14.9,10.9)$ & $6.40(\mathrm{dd}, 15.2,10.8)$ \\
\hline 10 & $6.22(\mathrm{dd}, 14.9,10.1)$ & $6.22(\mathrm{dd}, 14.9,10.8)$ \\
\hline 11 & $5.85(\mathrm{ddd}, 14.9,10.1,5.3)$ & $5.85(\mathrm{ddd}, 15.4,10.3,5.6)$ \\
\hline 12 & $\begin{array}{c}2.63(\mathrm{~m}) ; 2.24(\mathrm{ddd}, 13.7 \\
10.1,10.0)\end{array}$ & $2.62(\mathrm{~m}) ; 2.24(\mathrm{~m})$ \\
\hline 13 & $3.96(\mathrm{~m})$ & $3.92(\mathrm{~m})$ \\
\hline 14 & $1.68-1.59(\mathrm{~m})$ & $1.68-1.58(\mathrm{~m})$ \\
\hline 15 & $3.82(\mathrm{~m})$ & $3.80(\mathrm{~m})$ \\
\hline \multirow[t]{2}{*}{16} & $1.70(\mathrm{ddd}, 13.7,8.2,4.8)$ & 1.69 (ddd, 13.7, 8.6, 5.2) \\
\hline & $1.38(\mathrm{ddd}, 13.7,8.9,4.4)$ & 1.37 (ddd, $13.4,8.8,4.6)$ \\
\hline 17 & $3.89(\mathrm{~m})$ & $3.92(\mathrm{~m})$ \\
\hline \multirow{2}{*}{18} & $1.46(\mathrm{ddd}, 14.1,9.6,3.2)$ & $1.46(\mathrm{~m})$ \\
\hline & $1.20(\mathrm{ddd}, 14.1,10.1,2.4)$ & $1.19(\mathrm{ddd}, 14.2,10.5,2.7)$ \\
\hline 19 & $4.07(\mathrm{~m})$ & $4.05(\mathrm{~m})$ \\
\hline 20 & $1.33(\mathrm{~m}), 1.27(\mathrm{~m})$ & $1.32(\mathrm{~m}), 1.29(\mathrm{~m})$ \\
\hline 21 & $3.94(\mathrm{~m})$ & $3.92(\mathrm{~m})$ \\
\hline 22 & $1.33(\mathrm{~m}), 1.25(\mathrm{~m})$ & $1.32(\mathrm{~m}), 1.27(\mathrm{~m})$ \\
\hline 23 & $4.04(\mathrm{~m})$ & $4.05(\mathrm{~m})$ \\
\hline \multirow[t]{2}{*}{24} & $1.44(\mathrm{ddd}, 14.1,10.1,8.4)$ & $1.43(\mathrm{~m})$ \\
\hline & $1.33(\mathrm{~m})$ & $1.32(\mathrm{~m})$ \\
\hline 25 & $3.91(\mathrm{~m})$ & $3.92(\mathrm{~m})$ \\
\hline \multirow[t]{2}{*}{26} & $1.50(\mathrm{ddd}, 14.1,10.1,8.4)$ & $1.50(\mathrm{ddd}, 13.9,9.5,8.3)$ \\
\hline & $1.31(\mathrm{~m})$ & $1.32(\mathrm{~m})$ \\
\hline 27 & $4.22(\mathrm{~m})$ & $4.21(\mathrm{~m})$ \\
\hline 28 & $5.53(\mathrm{ddd}, 15.7,4.8,1.6)$ & $5.52(\mathrm{ddd}, 15.6,4.2,1.2)$ \\
\hline 29 & $5.64(\mathrm{ddd}, 15.7,5.2,1.2)$ & $5.63(\mathrm{ddd}, 15.6,5.2,1.0)$ \\
\hline 30 & $2.64(\mathrm{~m})$ & $2.64(\mathrm{~m})$ \\
\hline 31 & $4.78(\mathrm{dd}, 10.0,2.6)$ & $4.78(\mathrm{dd}, 9.9,2.6)$ \\
\hline 32 & $1.92(\mathrm{dqq}, 10.0,6.9,6.8)$ & $1.92(\mathrm{~m})$ \\
\hline 33 & $0.98(\mathrm{~d}, 6.9)$ & $0.97(\mathrm{~d}, 6.6)$ \\
\hline 34 & $1.06(\mathrm{~d}, 6.9)$ & $1.06(\mathrm{~d}, 7.0)$ \\
\hline 35 & $0.88(\mathrm{~d}, 6.8)$ & $0.88(\mathrm{~d}, 6.6)$ \\
\hline
\end{tabular}


II. Physicochemical properties

\begin{tabular}{|c|c|c|}
\hline Property & Natural & Synthetic \\
\hline Appearance & Yellow powder & Yellow powder \\
\hline $\mathrm{Mp}$ & $157-163{ }^{\circ} \mathrm{C}$ (dec.) & $159-160^{\circ} \mathrm{C}$ (dec.) \\
\hline Spec. Opt. Rot. & $-21^{\circ}(\mathrm{c}=0.3, \mathrm{MeOH})$ & $-21.7^{\circ} \mathrm{C}(\mathrm{c}=0.30, \mathrm{MeOH}$ \\
\hline HRMS & $\begin{array}{l}\text { Found: } 637.3930(\mathrm{M}+\mathrm{H}) \\
\text { Calcd: } 637.3951(\text { for } \\
\left.\mathrm{C}_{35} \mathrm{H}_{57} \mathrm{O}_{10}\right) \\
{[\mathrm{FAB}]}\end{array}$ & $\begin{array}{l}\text { Found: } 637.3957(\mathrm{M}+\mathrm{H}) \\
\text { Calcd: } 637.3952(\text { for } \\
\left.\mathrm{C}_{35} \mathrm{H}_{57} \mathrm{O}_{10}\right) \\
{[\mathrm{ESI}]}\end{array}$ \\
\hline $\mathrm{UV} \lambda_{\max }(\mathrm{nm})$ & 260,360 & 260,365 \\
\hline IR & $\begin{array}{c}3350,2900,1420,1250 \\
1105\end{array}$ & $\begin{array}{c}3368(\mathrm{~s}, \mathrm{br}), 2939(\mathrm{~m}), 1706 \\
(\mathrm{~m}), 1616(\mathrm{~m}), 1577(\mathrm{~m}), 1420 \\
(\mathrm{~m}), 1371(\mathrm{~m}), 1324(\mathrm{~m}), 1265 \\
(\mathrm{w}), 1237(\mathrm{w}), 1126(\mathrm{~s}), 1087 \\
(\mathrm{~m}), 1011(\mathrm{~m})\end{array}$ \\
\hline TLC & $\begin{array}{c}0.45 \text { (silica gel, } \\
\left.\mathrm{CHCl}_{3} / \mathrm{MeOH} 2 / 1\right)\end{array}$ & $\begin{array}{c}0.41 \text { (silica gel, } \\
\mathrm{CHCl}_{3} / \mathrm{MeOH} 2 / 1 \text { ) }\end{array}$ \\
\hline HPLC & $\begin{array}{c}\text { Senshu Pak ODS-H } \\
80 / 20 \mathrm{MeOH} / \mathrm{H}_{2} \mathrm{O} \\
\text { flow }=6.0 \mathrm{~mL} / \mathrm{min} \\
\text { detector } 200-400 \mathrm{~nm} \text { array } \\
\mathrm{t}_{\mathrm{R}}=28 \mathrm{~min}\end{array}$ & $\begin{array}{c}\text { Vydac C-18 } \\
60 / 40-80 / 20 \mathrm{MeOH} / \mathrm{H}_{2} \mathrm{O} \\
\text { flow }=8.0 \mathrm{~mL} / \mathrm{min} \\
\text { detector } 360 \mathrm{~nm} \\
\mathrm{t}_{\mathrm{R}}=43 \mathrm{~min}\end{array}$ \\
\hline $\mathrm{CD}$ & & $\begin{array}{c}\text { negative Cotton effect } \\
\text { peak at } 210 \mathrm{~nm}(10 \mathrm{mdeg}) \\
\text { trough at } 257 \mathrm{~nm}(-7 \mathrm{mdeg}) \\
(0.5 \mu \mathrm{M} \text { in } \mathrm{MeOH})\end{array}$ \\
\hline
\end{tabular}




\section{References}

(1) Still, W. C.; Kahn, M.; Mitra, A. J. Org. Chem. 1978, 43, 2923.

(2) Gilman, H.; Cartledge, F. K. J. Organometal. Chem. 1964, 2, 447.

(3) Karaev, S. F.; Kuliev, R. M.; Guseinov, Sh. O.; Askerov, M. E.; Movsumzade, M. M. J. Gen. Chem. USSR., 1982, 52, 1016.

(4) Denmark, S. E.; Beutner, G. L. J. Am. Chem. Soc. 2003, 125, 7800.

(5) Evans, D. A.; Dart, M. J.; Duffy, J. L.; Yang, M. G. J. Am. Chem. Soc. 1996, 118, 4322.

(6) Denmark, S. E.; Tymonko, S. A. Manuscript submitted

(7) Rossi, R.; Bellina, F.; Catanese, A.; Mannina, L.; Valensin, D. Tetrahedron 2000, 56, 479.

(8) (a) Takeuchi, R.; Tanabe, K.; Tanaka, S. J. Org. Chem. 2000, 65, 1558. (b) Dixon, D. J.; Ley, S. V.; Longbottom, D. A. Org. Synth. 2003, 80, 129.

(9) Mori, Y.; Asai, M.; Kawabe, J.; Furukawa, H. Tetrahedron 1995, 51, 5315. 